\title{
FLORA AND FAUNA
}

WITHIN THIRTY MILES OF HANOVER, N. H.

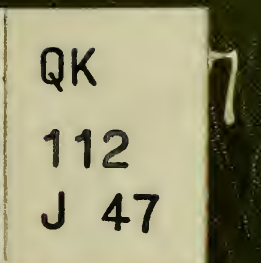





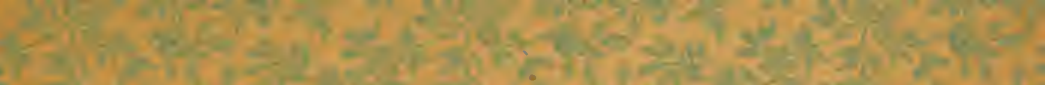

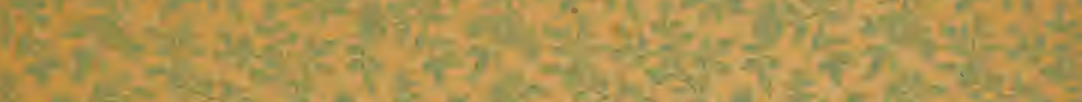

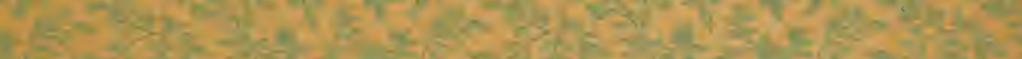

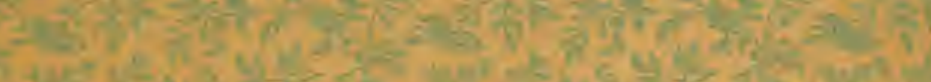

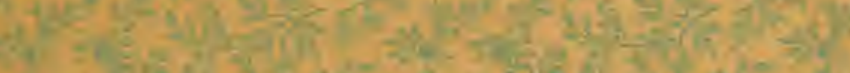
स)

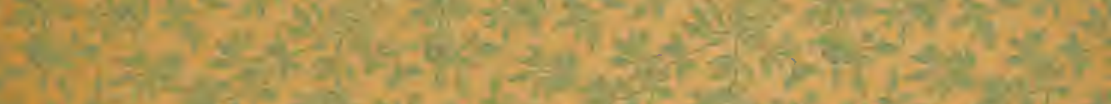
S.

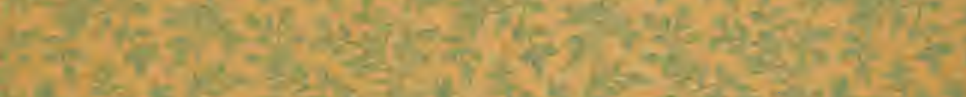

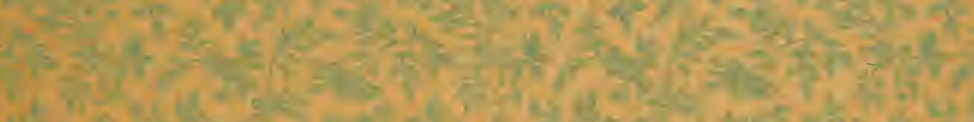

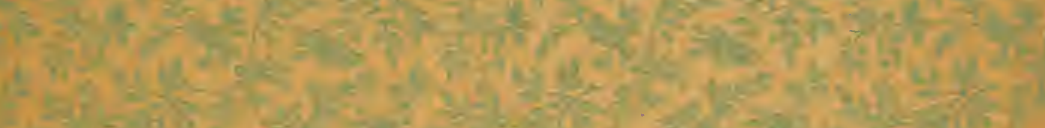

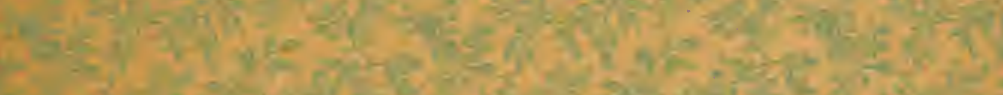

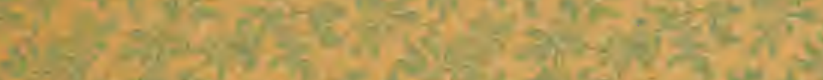

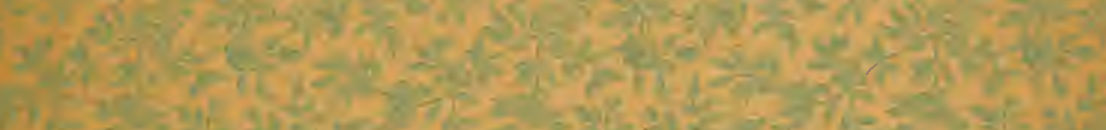

H.

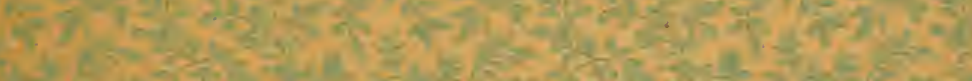

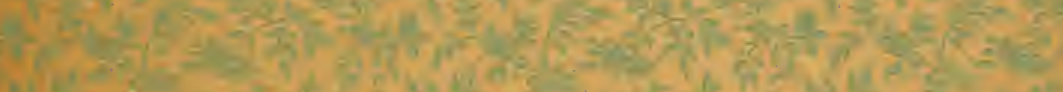

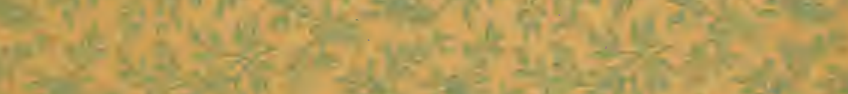

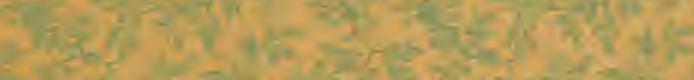

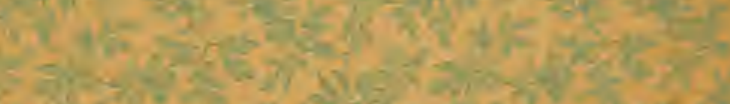
H.

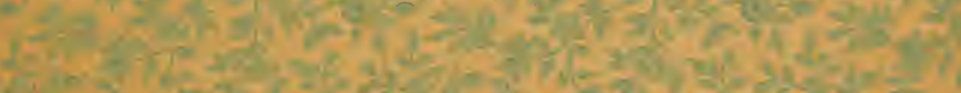

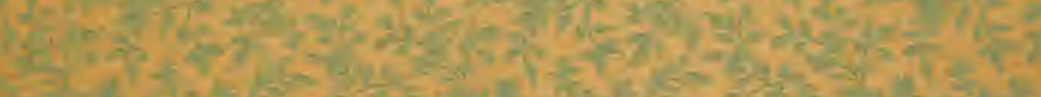

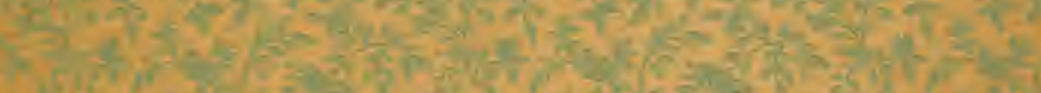

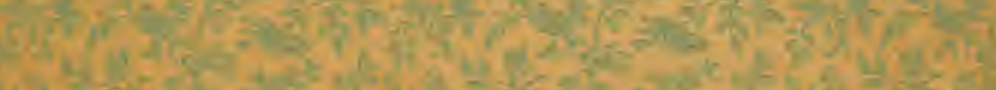

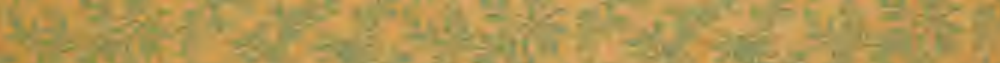

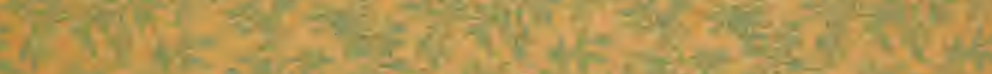

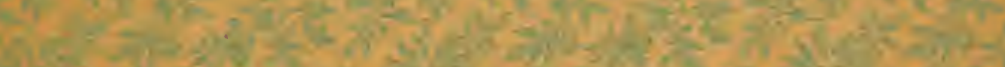

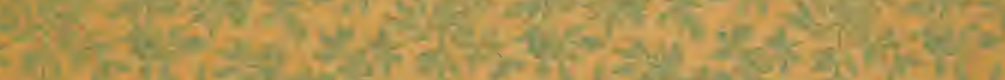

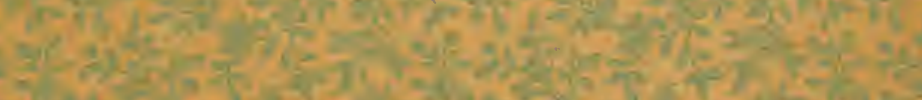

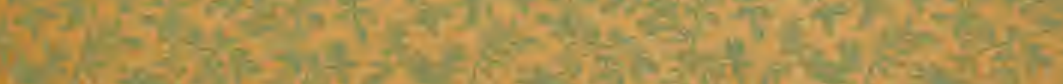

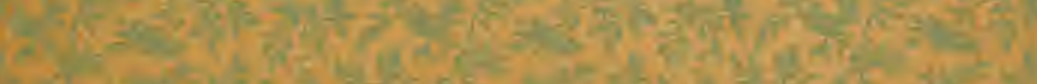

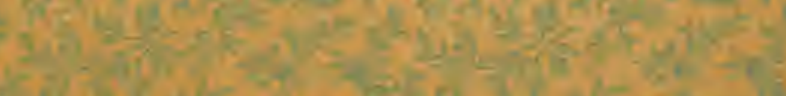

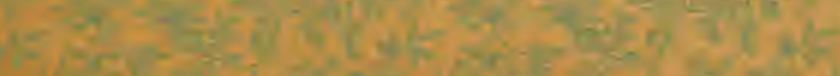





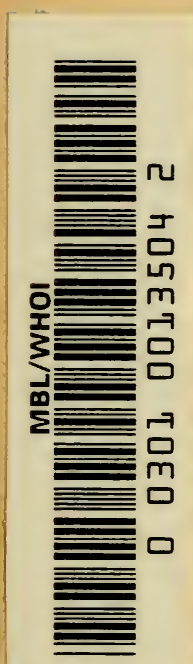





\section{A CATALOGUE}

OF THE

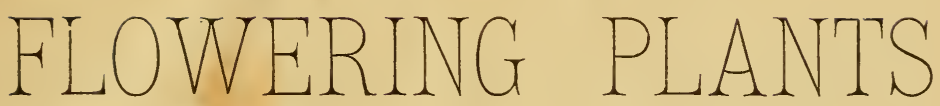

AND

\section{HIGHER CRYPTOGAMS,}

Both Native ANd INtroduced, Found within about thirty miles of Haxover, N. H., including a Few CultiVATED SPECIES, to Which Is APPENDED

A List of Vertebrate Animals of the Same Region.

BY

HENRY G. JESUP, A. M.,

Professor of Natural History.

PRINTED FOR THE AU'THOR.

1891. 


\section{Directions for Collecting and Preserving Plants and for the Formation of an Herbarium.}

I. A complete specimen will represent the root, stem, leaves, flowers, and fruit.

2. Collect small plants, like the Violet, entire.

3. When the leaves of the stem and the root differ, as in some Buttercups, get both.

4. If the staminate and the pistillate flowers are on different plants, as in Thalictrum (Rue), or on different parts of the same plant, as in the Butternut, look for both.

5. Plants of the Mustard Family (Cruciferæ) and the Parsley Family (Umbelliferæ), with all Rushes and Sedges, are classified by the fruit. Grasses may be gathered in flower.

6. Ferns should be in fruit, and when the sterile and fertile fronds differ, as in Osmunda, get both.

7. Thick roots and stems can be split in two, and one half only be preserved.

8. Arrange the plant to be drier in a folded sheet of thin, bibulous paper, and do not disturb it until dry. Let thick pads of drying paper alternate with these single sheets, and then place the whole under pressure. Change the drying pads every day or two, and dry as rapidly as practicable.

9. These dried plants attached to sheets of paper by strips of surgeon's plaster or by glue, fully ticketed with name, when and where and by whom collected, and arranged in Orders and Genera as in the Manual, constitute an Herbarium.

\section{Summary of Species.}

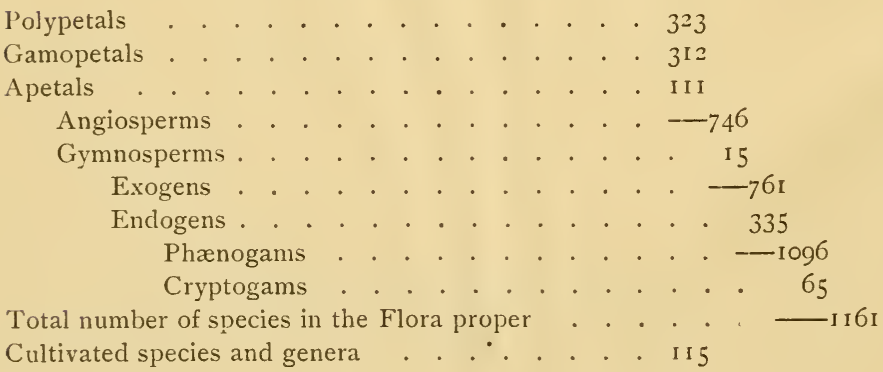

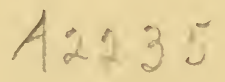




\section{PRE F A CE.}

The following Catalogue is intended to include, so far as known, the Flora within about thirty miles radius of the town of Hanover, New Hampshire, a circuit extending to and embracing the mountain peaks Moosilauke and Kearsarge in New Hampshire, and Killington in Vermont. The outlying towns intersected by the circumference of the circle, beginning with the southern limit and the Connecticut river, are, in New Hampshire, Charlestown, Acworth, Lempster, Goshen, Newbury, Sutton, Andover, Hill, New Hampton, Bristol, Bridgewater, Plymouth, Campton, Ellsworth, Benton, and Haverhill. Continuing the circuit on the Vermont side of the river, the towns are Newbury, Topsham, Orange, Williamstown, Brookfield, Braintree, Rochester, Pittsfield, Chittenden, Mendon, Shrewsbury, Mit. Holly', Ludlow, Chester, and Springfield. The region thus indicated is divided nearly equally between the two adjoining states by their common boundary the Connecticut river, and includes a variety of soil, climate, and location sufficient to furnish a home for a majority of the plants of northern New England, except those peculiar to the seashore and the higher mountains.

The only river of any considerable size is the Connecticut, running from north to south throughout the entire district, a distance of sixty miles or more. Numerous tributaries join this river on both sides, the largest of which, in Vermont, are the Ompompanoosuc which empties in Norwich, the White river at White River Junction, the Ottoquechee in Hartland, a few miles from whose mouth is the deep gorge known as "Quechee Gulf," and the Black river in Springfield. In New Hampshire, the Mascoma, the outlet of Mascoma Lake, joins the Connecticut in West Lebanon, and the Sugar river in Claremont. The Pemigewasset and other streams which form the head waters of the Merrimack river, moreover, reach some towns in the eastern part of the district.

The larger sheets of water included are, in New Hampshire, Sunapee lake, Newfound lake, and Mascoma lake, or Enfield pond. Sunapee lake, in the towns of Sunapee, Newbury, and New London, is about seven miles long, and at one point two and one half miles broad, and is a well known summer resort. Newfound lake in the towns of Hebron, Bridgewater, and Bristol, is less known, but is a fine sheet of water, six miles perhaps in length, and three miles in extreme breadth, and very nearly as large as Sunapee. Mascoma lake or Enfield pond is somewhat smaller, being four and a half miles long by rather more than half a mile in width.

On the Vermont side there is little to correspond with the above. Fairlee pond or Morey lake in the town of Fairlee, and Fairlee lake, partly in the same town, are both comparatively small. The purity of the water in these larger lakes, and their conımonly bold or gravelly shores, are not favorable to the accumulation of much aquatic vegetation, but even here the botanist will 
find some plants, like Bidens Beckii (Water Marigold) for instance, not found elsewhere, and all these lakes will repay exploration. It is the smaller ponds and adjoining marshes, rich with decaying vegetation, that more abundantly reward the persevering herbalist. Of these, also, there are more on the eastern side of the Connecticut river than on the western side. Not a few of them are high up among the hills, visited very seldom and by very few, but in these high and cool bogs there still is found the remains of a vegetation that long since deserted the warmer lowlands. Here are the rarer Sedges and Grasses, Sarracenia (Pitcher Plant), and Orchids, besides Ericaceous plants like Azalea, Rhodora, Andromeda, and Ledum (Labrador Tea), as well as others of equal interest. Periods of drought seldom reach these rich and moist garden spots.

Of the three mountain peaks referred to above, Moosilauke in Benton, N. H., $4,8 \mathrm{I}$ ft. in altitude, is the only one having a treeless summit of any considerable extent, suggestive of Alpine plants, and there a few species appear, such as Arenaria Granlandica (Mountain Sandwort), Loiseleuria procumbens (Alpine Azalea), Vaccinium Vitis-Idca (Mountain Cranberry), Vaccinium uliginosum (Bog Bilberry), and Solidago alpina (Alpine Golden-rod), etc. Kearsarge Mt. near Salisbury, N. H., and Killington Mt., with the adjoining peaks in Sherburne and Mendon, Vt., are well wooded nearly to their summits, and have upon them little or nothing strictly alpine. The same is true of Mt. Ascutney near Windsor, Vt., and the same may be said of Cube Mt. in Orford, and Smart's Mt. in Lyme, N. H. This latter elevation is peculiar in this, that the timber on the summit, instead of being stunted and worthless, is of full marketable size. Cardigan Mt. in Orange, N. H., has a conspicuous treeless summit, but little truly Alpine, unless we except Vaccinizm Vitis-Idaa, has been reported from there. It is probable that a more careful exploration might result in some new discoveries on any or all of these mountains.

In addition to the lakes and mountains, numerous streams are found within the district on whose banks and in whose waters are forms of vegetation peculiar to such localities and to them alone. Rivers like the Connecticut, with numerous tributaries, are great highways for the distribution of both seeds and growing plants. Every freshet bears these along in its swift current, and transports them many miles from the place of their origin. They are stranded on the shore, or find a home in the still pools along the margin of the stream, and once established may remain indefinitely. This explains the presence of Astragalus alpinus (Alpine-Vetch) on the rocks at Sumner's Falls in Plainfield, N. H.-a plant of the far north-together with Astragalus Robbinsii, a species of equal interest. When, moreover, the river recedes during a period of drought, the shore that is laid bare supplies conditions under which a still different vegetation makes its appearance. Ranunculus Flammula, var. reptans (Creeping Buttercup) then spreads its green carpet dotted with yellow stars upon the sand; and other plants, that are shut out of their needed supply of oxygen by high water, begin once more to flourish.

Plants are as migratory as any other of the world's inhabitants. They are always ready to make use of the same great thoroughfares and of the same 
means of transportation. Every new railroad or canal or highway opens for them a direct line of communication with regions not yet visited, and they instantly make use of them all. Every steamship or sailing vessel, whether from across the ocean or coasting along our own shores, aids in the distribution, while all these and similar agencies are but supplementary to those which, entirely apart from civilization, are in constant operation.

The early inhabitants of New England had scarcely landed on our shores before they found that the Ox-eye Daisy and the Dandelion, together with the Buttercups and Docks and Plantains and a score of similar emigrants from the old world, had accompanied them. And when the tide of trave] advanced westward toward the Mississippi river, not only did these same plants follow the same route, but in due time the plants of the prairie, like Rudbeckia hirta (Yellow Daisy or Cone-flower) began to appear in Eastern meadows. These introduced plants are sometimes of great value, like the Grasses and Clovers, but commonly they are the so called weeds of the farm and the garlen, and come unbidden and unwelcome. Some come to stay, others that appear occasionally about our manufacturing establishments, persist for a time only, but finally disappear to be followed very likely by a new importation. Plants of this kind are in some respects more interesting than those of our native Flora, not only because we come more directly in contact with them, but because each has a history behind it and around it, which serves to illustrate the begimnings and the development of that portion of the country where it has found a new home.

The fields and open woodlands, the roadsides and fence rows, are naturally first visited by the plant collector because easily accessible, but every nook and corner, whether in sunshine or in shade, in its season, has its own share of vegetable as well as animal life, and the lover of Nature who would become thoroughly acquainted with her treasures seeks them everywhere, for the simple reason that they may be found everywhere. The Flora of no section of our country will ever be known so completely that nothing more shall remain to be learned about it. No surprising discoveries of new species may be made, though of this we dare not be too sure, but new chapters in the history of well known plants may be opened, newly arrived immigrants noted, new varieties found, new facts as to distribution and new economical uses ascertained, as well as new relations between the species and its environment in illustration of the conflict all plants are making successfully or unsuccessfully for an existence. For reasons like these a region which has been as long settled and is as well known as the one embraced in this Catalogue will never be without interest to the naturalist.

The relation of what is here called the Hanover Flora to the general New England Flora calls for some brief notice. Thirty miles south of Hanover, in the towns of Charlestown, N. H., and Springfield, V't., some of the trees and shrubs so common a little farther south in both of these states have already reached their northern limit, and begin to disappear from the native woods. The Chestnut and the Shagbark Hickory are no longer abundant. The season is too short for any considerable quantity of fruit to reach perfection, and seedlings are rare. Trees of these two kinds when planted in sheltered localities 
may attain considerable age and size. A single chestnut of exceptional size may be seen in Norwich, Vt., five feet eight inches in diameter, and more than fifty years old, but it was planted where it now stands, and the chestnuts are of little value. An occasional Shagbark Hickory of moderate size may also be met with, though the Pignut Hickory is not infrequent in the woods, and the Bitternut Hickory ascends the Connecticut as far north as Wells river. The Black Birch, and the Grey Birch, as well as the Pitch Pine, are rare north of Hanover and not common in its vicinity, while the Sycamore or Buttonwood is nowhere seen in a wild state. To the above may be added as decidedly rare, the Sassafras, the Spice Bush (Lindera), the Flowering Dogwood (Cormus florida), the Red Cedar, and the common Juniper, together with many other trees and shrubs that fifty miles south of Hanover may be common.

On the other hand, as the traveller advances thirty miles northward from Ilanover, another class of aborescent vegetation appears, that either is not found at all southward, or at least is not abundant. Here the White Cedar becomes plentiful and very valuable, the Tamarack or American Larch fills the swamps, and the White Spruce occasionally appears, while the Black Spruce covers the lower mountain tops, the Paper Birch adorns the hillsides, the Green Alder is found by the streams and in the ravines, and the Elder has red berries more commonly than black ones. Additional illustrations might readily be given, but the above will indicate what changes may be noted within sixty miles only of latitude.

The first and preliminary edition of this Catalogue was issued in $\mathrm{r}_{8 \mathrm{~S}}$. Since that time some additional explorations of the region have been made, very much increasing the list of names and supplying much valuable information.

In order to make the Catalogue as useful as possible to the author's classes in Natural History, for whom primarily it has been prepared, there have been added the names of such plants as are most commonly met with in cultivation, arranged under their respective Orders. These do not properly belong to the Flora, and are distinguished from the rest either by the arrangement of the type or by being enclosed in square brackets. Their description will be found in Gray's Field, Forest, and Garden Botany, while the description of plants belonging to the Flora proper will be found in Gray's Manual of the Botany of the Northern United States, Sixth Edition, 1890. The synonyms refer to the fifth edition of the same Manual. The names of introduced and cultivated species are printed in SMALL CAPITALS. The local or English name of each plant has been added so far as known. And as an additional aid to the student, brief directions have been given for collecting and preserving plants and for the formation of an Herbarium. Localities not designated are in New Hampshire.

In the preparation of the previous edition of the Flora, free use was made of a Catalogue of New Hampshire plants compiled for the Geological Survey of the State by Wm. F. Flint, and a large amount of information and personal assistance was contributed by Nathan Barrows, M. D., then of Meriden. From the Vermont side of the Connecticut river some rare species were 
reported from North Pomfret by Prof. A. P. Morgan; from Sharon by E. K. Baxter, M. D.; and from Union Village by Ferdinand Blanchard, M. D.; while Mt. Ascutney and the vicinity of Windsor were thoroughly explored by George H. Leland.

For the present edition it has been found necessary to rewrite and rearrange a large part of the Flora to correspond with the changes in both classification and nomenclature found in the last edition of the Manual referred to above. As the work has been in progress, important aid has generously been furnished by the author's numerous friends and correspondents in various parts of the country-too numerous to be mentioned individually-and he would here convey to them his hearty thanks. The students who year by year have been connected with his classes have done their share also in bringing this Flora to its present completeness. The names of very many of these contributors, both correspondents and students, will be found in their appropriate places in the following pages.

The List of Vertebrate Animals found within the same district as the Flora, originally given in the edition of ISS2, was prepared by Prof. T. W. D. Worthen, with the assistance of Prof. B. T. Blanpied and Prof. C. S. Cook, and was intended for use with Jordan's Vertebrates of the Northern United States as published in ISSo.

The List here given has also been prepared by Prof. Worthen who has embodied his own notes made since the previous edition was issued, as well as some additional information furnished by other naturalists, residents now or of late in this vicinity, whose names are given in connection with the items reported by them. To Prof. Worthen and to these gentlemen cordial thanks are rendered.

The classification adopted is that of the Fifth and Revised Edition of Fordan's Vertebrates of I $8 S 8$, involving very many changes in both names and general arrangement. The above book must therefore be consulted for full descriptions.

The accompanying map, contributed by the students, indicates clearly the region represented by the Flora and Fauna.

Additions and corrections are solicited, and will always be welcome.

H. G. J.

Hanover, N. H., Jan. 23, IS9r. 



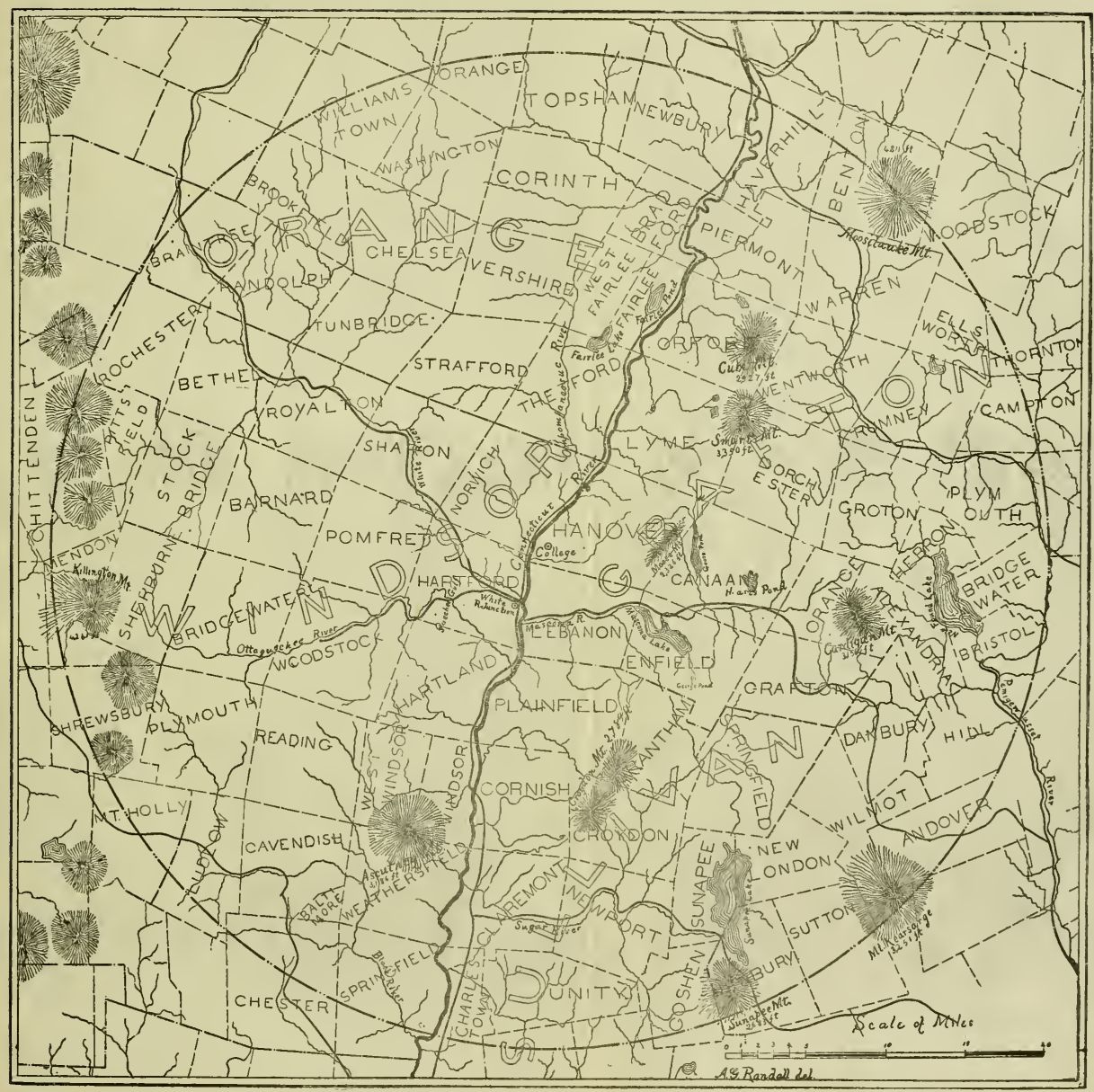

MAP OF THE REGION WITHIN THIRTY MILES OF HANOVER, N. H. 



\title{
CATALOGUE OF PLANTS.
}

\author{
SERIES 1. \\ PHENOGAMOUS OR FLOWERING PLANTS.
}

Class I. Dicotyledonous or EXOGENOUS PLANTS.

SUBCLASS I. ANGIOSPERMÆ.

Division I. POLYPETALOUS EXOGENS.

Order i. RANUNCULACE无. Croinfoot Family.

Clematis, L. Virgin's Bower.

C. Virginiana, L. River-banks, etc.; common.

C. verticillaris, DC. Open woods. Windsor, Vt., G. H. Leland; Hartland, Vt., W. W. Eggleston; Lyme, N. H., Z. A. Norris; rare.

Anemone, Tourn. IVind-Flower. Anemone.

A. cylindrica, Gray. Pomfret, Windsor, and Norwich, Vt.; not abundant.

A. Virginiana, L. Woods and roadsides; common.

A. nemorosa, L. Margin of woods; common.

Hepatica, Dill. Liver-leaf. Liverwort.

H. triloba, Chaix. Woods. More common southward.

H. acutiloba, DC. Woods. The more abundant species.

Anemonella, Spach. Rue-Anemone.

A. thalictroides, Spach. (Thalictrum anemonoides, Michx.) Claremont, Dr. N. Barrows; rare.

Thalictrum, Tourn. Meadow-Rue.

T. dioicum, L. (Early-Rue.) Rocky woods; common.

T. polygamum, Muhl. (T. Cornuti, Gray's Manual, 5th ed.)-(Late-Rue.)

Wet meadows; common. 
Ranunculus, Tourn. Crowfoot. Buttercup.

R. circinatus, Sibth. ( $\mathrm{R}$ divaricatus, Manual, 5th ed.)-(White WaterCrowfoot.) Cold streams and ponds. Windsor, Vt., Leland; rare.

R. aquatilis, L., var. trichophyllus, Gray. (White Water-Crowfoot.) Slow streams. Dr. Barrozus; rare.

R. multifidus, Pursh. (Yellow Water-Crowfoot.) Hanover, N. H.; Norwich, Vt.; rare.

R. Flammula, L., var. reptans, E. Meyer. (Small Creeping-Crowfoot) Sandy shore of Conn. river; shore of pond, New London, H. E. Sargent.

R. abortivus, L. (Small-flowered Crowfont.) Common.

R. sceleratus, L. North Charlestown, Dr. Graves; rare.

R. recurvatus, Poir. (Hooked Crowfoot.) Common.

R. fascicularis, Muhl (Early C.) North Charlestown, Dr. Graves.

R. septentrionalis, Poir. (R. repens, Manual, 5th ed.)-(Large Creeping-Crowfoot.) Wet meadows; common.

R. Pennsylvanicus, L. (Bristly Crowfoot.) Wet places; common.

R. Bulbosus, L. Hanover, F. P. Comings; Meriden, Dr. Barrows; Norwich, Vt., Dr. Blanchard; Chelsea, Vt., F. M. Comstock.

R. ACRIS, L. Common Buttercup. This and the above, European weeds.

Caltha, L. Marsh-Marigold. Cowslips.

C. palustris, L. Hanover and Lebanon, N. H.; Norwich, Vt.; etc.; common.

Coptis, Salisb. Gold-thread.

C. trifolia, Salisb. Wet woods; common.

Aquilegia, Tourn. Columbine.

A. Canadensis, L. Rocks; common.

Cimicifuga, L. Bugbane.

C. racemosa, Nutt. (Black Cohosh.) Claremont, Dr. Barrows; very rare.

Actæa, L. Baneberry.

A. spicata, L., var. rubra, Ait. (Red Baneberry.) Rich woods; common. A. alba, Bigel. (White Baneberry.) A little later in flowering.

[Among cultivated plants of this Order are various species of ANEMONE, Clematis, Aquilegia (Columbine), Delphinium (Larkspur), Aconitum (Monkshood), PÆonia (Pæony), Ranunculus, etc.]

Order 2. MENISPERMACE开. MoONSEed FAMily.

Menispermum, L. Moonseed.

M. Canadense, L. Claremont, both sides Conn. river. Dr. Barrows.

Order 3. BERBERIDACE开. BARberRy FAMily.

Berberis, L. Barberry.

B. vULGaRIS, L. More commonly cultivated than wild; from Europe.

Caulophyllum, Michx. Blue Cohosh.

C. thalictroides, Michx. Rich woods; common. 
Podophyllum, L. Mandrake.

P. peltatum, L. Hanover, N. H.; Norwich, Vt., E. A. Mack; doubtless introduced.

ORDER 4. NYMPH仺ACE开. WAter-Lily Family.

Brasenia, Schreber. Water-shield.

B. peltata, Pursh. In ponds.

Nymphæa, Tourn. White Water-Lily.

N. odorata, Ait. In ponds ; common.

Nuphar, Smith. Yellow Pond-Lily.

N. advena, Ait. (Cow Lily.) Everywhere common.

var. minus, Morong. (N. rubrodiscum, Morong.) Squam Lake, Fesup. A hybrid, probably, between N. advena and the next species; intermediate in size, with stigmas crenately toothed and bright red or crimson.

N. Kalmianum, Ait. (N. luteum, var. pumilum. Man., 5th ed.) Hanover, N. H.; Dr. Barrows ; Sherburne, Vt., W.W. Eggleston.

ORder 5. SARRACENIACE开. PIrCher-Plant Family.

Sarracenia, Tourn. Pitcher-Plant. Side-Saddle Flower.

S. purpurea, L. Lebanon, Meriden, and New London, N. H.; Norwich and . Sharon, Vt. In peat bogs; not rare.

Order 6. PAPAVERACE无. Poppy Family.

Sanguinaria, Dill. Blood-root.

S. Canadensis, L. Borders of rich woods; common.

Chelidonium, L. Celandine.

C. majus, L. Near dwellings and fences; from Europe.

ORder 7. FUMARIACE无. Fumitory Family.

Adlumia, Raf. Mountain Fringe. Climbing Fumitory.

A. cirrhosa, Raf. Claremont and Plainfield, N. H. ; Ludlow, Windsor, and Royalton, Vt.; not common; often cultivated.

Dicentra, Borkh. Dicentra.

D. Cucullaria, DC. (Dutchman's Breeches.) Rich woods; common.

I). Canadensis, DC. (Squirrel-Corn.) Rich woods; not rare.

[D. eximia, DC., from Va. is frequent in old gardens.]

Corydalis, Vent.

C. glauca, Pursh. (Pale Corydalis.) Rocky places and clearings.

C. aurea, Willd. (Golden Corydalis.) Norwich, Vt., near the Passumpsic R. R. Fesup.

Fumaria, Tourn. Fumitory.

F. officinalis, L. A weed in gardens; European. 


\section{Order S. CRUCIFER丑. Mustard Family,}

Dentaria, Tourn. Pepper-root.

D. diphylla, L. Rich woods; common.

D. laciniata, Muhl. Plainfield, Dr. Barrozes; rare.

D. maxima, Nutt. With the first, but less common.

Cardamine, Tourn. Bitter Cress.

C. hirsuta, L. IVet ground. A slender variety is found in dry places.

Arabis, L. Rock-Cress.

A. hirsuta, Scop. Rocky places; not rare.

A. lævigata, Poir. WVoods and ravines; not common.

A. perfoliata, Lam. (Tower Mustard.) Hartford, Vt., Flint; Norwich, Vt., Fesup; Hanover, Lyme, etc.; not common.

A. confinis, Watson. (A. Drummondii, Manual, 5th ed.) Hanover, N. H., A. C. French ; Fairlee, Vt., F. O. Grover; not common.

Camelina, Crantz. False Flax.

C. SAtiva, Crantz. Occasionally in grass grounds. European.

Nasturtium. R. Br. Water-Cress.

N. Sylvestre, R. Br. (Yellow Cress.) A weed in river meadows. Norwich, Vt.; etc. ; from Europe.

N. palustre, DC. Marsh Cress. WVet places; common.

N. Armoracia, Fries. (Horseradish.) Rarely seen in fruit; European.

Barbarea, R. Br. Winter Cress. Yellow Rocket.

B. vulgaris, R. Br. Low grounds; common.

Hesperis, Tourn. Common Rocket.

H. matronalis, L. Roadsides, etc. ; a garden escape.

Erysimum, Tourn. Treacle Mustard.

E. cheiranthoides, L. Hanover; a weed in gardens.

Sisymbrium, Tourn. Hedge Mustard.

S. Offiçinale, Scop. Common European weed.

Brassica, Tourn. Cabbage, Mustard, etc.

B. CAMPESTRIS, L. (Kale.) Common in grain fields; a troublesome weed.

B. Sinapistrum, Boissier. (English Charlock.) Plainfield, Miss Stevens ; Plymouth, H. E. Sargent ; not common.

B. ALbA, Gray. (White Mustard.) Rare.

B. NIGRA, Koch. (Black Mustard.) Common near dwellings; all from Europe.

Capsella, Medic. Shepherd's Purse.

C. Bursa-PASTORIS, Moench. A weed in gardens; from Europe.

Thlaspi, Tourn. Pennycress.

T. Arvense, L. Pomfret, Vt., A. P. Morgan; Lebanon, Fesup; waste places; rare; European. 
Lepidium, Tourn. Peppergrass.

L. Virginicum, L. Roadsides; common.

L. Campestre, Br. Hanover, C. H. Dana: not common; European.

L. RUderale, L. Hanover, etc. ; along with L. Virginicum ; from Europe.

Raphanus, Tourn. Wild Radish.

R. RAPHANistrum, L. (Jointed Charlock.) Hanover; not rare in grain fields; European.

[Cultivated plants:-Various kinds of BRASSICA (Cabbage and Turnip), Raphanus sativum (Garden Radish), Lepidium sativum (Garden Peppergrass), MatTHIOla (Stock), Iberis (Candytuft), Alyssum MaRitimuM (Sweet Alyssum), etc.]

Order 9. CISTACE无. Rock-Rose FAMily.

Helianthemum, Tourn. Rock-Rose.

H. Canadense, Michx. Frost-weed. Gravelly soil.

Lechea, Kalm. Pinweed.

L. major, Michx. Dry and sterile fields ; common.

L. minor, L. With the above and quite variable, including perhaps more than one species.

\section{Order io. VIOLACE无. V'tolet Family.}

Viola, Tourn. Violet.

V. palmata, L. (V. cucullata, var. palmata. Gray.)-(Palmately-leaved Violet.) On drier ground than the variety, and less common.

var. cucullata, Gray. (V. cucullata, Ait.)-(Common Blue Violet.) Very common everywhere.

V. sagittata, Ait. Hanover, etc. Roadsides and upland pastures; not abundant.

V. Selkirkii, Pursh. (Selkirk Violet.) Shady hillsides; not very common.

V. blanda, Willd. (Sweet White Violet.) Moist places; common, and very variable.

var. palustriformis, Gray. "The larger form ; in shady, mossy ground." var. renifolia, Gray. (Kidney-leaved Violet.) Named from specimens collected in 1870 by $F$. A. Sherman : common in rich, rocky woods.

V. primulafolia, L. (Primrose-leaved Violet.) With the next. New London, $W$. F. Carter; not common.

V. lanceolata, L. (Lance-leaved Violet.) Near Dorchester Line Pond, A. C. French; New London, in wet pastures, Carter; rare.

V. rotundifolia, Michx. (Round-leaved Violet.) Moist woods. The earli est species ; common.

V. pubescens, Ait. (Downy Yellow Violet.) Woods; common and variable.

V. Canadensis, L. (Canada Violet.) Rich woods; common.

V. rostrata, Pursh. (Long-spurred Violet.) With the above.

V. canina, L. var. Muhlenbergii, Gray. (Uog Violet.) Everywhere common and rather variable. 
V. TRICOLOR, L. (Pansy.) Spontaneous in gardens along with V. odorATA, the English Sweet Violet.

Order it. CARYOPHYLLACE无. Pink FAMILy.

Dianthus, L. Pink.

D. Arieria, L. (Deptford Pink.) Randolph, Vt., Bates.

D. DEltoides, L. (Maiden Pink.) Near Sharon, Vt. ; well established on the roadside, $\mathcal{F}$ esup.

Saponaria, L. Soapwort. Bouncing Bet.

S. officinalis, L. Roadsides; commonly double; European.

Silene, L. Catchfly.

S. Cucubalus, Wibel. (S. inflata, Smith.)-(Bladder Campion.) Meriden and Hanover, N. H. ; Chelsea, Vt. ; etc. Roadsides; European.

S. antirrhina, L. (Sleepy Catchfly.) IVaste places.

S. NOCTIFLORA, L. Frequent; introduced with grass seed.

[S. ARmeria, L. (Sweet-William Catchfly.) Spontaneous in gardens.]

Lychnis, Tourn. Lychnis.

L. Githago, Lam. (Corn-cockle.) Hanover, in cultivated fields; from Europe.

Arenaria, L. Sandwort.

A. Serpyllifolia, L. IVindsor, Vt., Leland. IVaste places; European.

A. Michauxii, Hook. (A. stricta, Michx.) Norwich, Vt., Dr. Blanchard; Fairlee Cliffs, Vt., F. O. Grover. Not very common.

A. Grœnlandica, Spreng. (Mountain Sandwort.) Moosilauke Mt., W. H. Flint; Kearsarge Mt., Dr. Barrozus.

A. lateriflora, L. Lebanon, at Olcott Falls ; infrequent.

Stellaria, L. Chickweed.

S. MEdia, Smith. (Common Chickweed.) From Europe.

S. longifolia, Muhl. (Long-leaved Stitchwort.) Grassy places.

S. longipes, Goldie. Claremont, Dr. Barrows; rare.

S. GRAMINEA, L. Occasional in cultivated grounds. European.

S. borealis, Bigel. WVet, shady places; not rare.

S. uliginosa, Murr. (Swamp Chickweed.) Rochester, Vt., Eggleston.

Cerastium, L. Chickweed.

C. vulgatum, L. (C. viscosum of Gray's Man., 5th ed.) Common European weed.

C. arvense, L. Rocky pastures north of IVindsor, Vt., Flint; Hanover, N. H., G. M. Moore; scarce.

Sagina, L. Pearlwort.

S. procumbens, L. Moist places.

Buda, Adans. Sand-Spurrey.

B. rubra, Dumort. (Spergularia rubra. Presl.) Dry, sandy soil ; Canaan and Claremont, N. H., Dr. Barrows ; Royalton, Vt., H. Q. Ward; Plymouth and New London, N. H., Sargent. 
Spergula, L. Spurrey.

S. ARvensis, L. (Corn-Spurrey.) Hanover, Meriden, etc.; in cultivated grounds; European.

Order i2. PORTULACACEÆ⿸尹 Purslane Family.

Portulaca, Tourn. Purslane.

P. OLERACEA, L. A common weed in gardens; European.

Claytonia, Gronov. Spring-Beauty.

C. Caroliniana, Michx. Common in open woods.

ORDER I3. HYPERICACE无. St. John's-Wort FAMILy.

Hypericum, Tourn. St. John's-wort.

H. Ascyron, L. (H. pyramidatum, Ait.)-(Great St. John's-wort.) Lebanon,

N. H.; Pomfret, Windsor, and Royalton, Vt. ; not common.

H. ellipticum, Hook. Wet places.

H. perforatum, L. (Common St. John's-wort.) Fields; European.

H. maculatum, Walt. (H. corymbosum, Muhl.) Damp places; common.

H. mutilum, L. Damp places.

H. Canadense, L. Very common in sandy soil. var. majus, Gray. Not uncommon.

H. nudicaule, Walt. (H. Sarothra, Michx.)-(Orange Grass.) Sandy fields; common.

Elodes, Adans. Marsh St. John's-wort.

E. campanulata, Pursh. (E. Virginica, Nutt.) Swamps.

ORDER I4. MALVACE无. Mallow Family.

Malva, L. Mallows.

M. Rotundifolia, L. (Common Mallow.) Near dwellings.

M. CRISPA, L. (Curled Mallow.) Occasionally seen on the roadsides.

II. moschata, L. (Musk Mallow.) Roadsides and fields.

M. sylvestris, L. (High Mallow.) Old gardens; all European.

Abutilon, Tourn. Indian Mallow.

A. Avicennæ, Gærtn. (Velvet-Leaf.) Roadsides and waste heaps; from India.

Hibiscus, L. Rose-Mallow.

[H. Trionum, L. (Flower-of-an-hour.) State Farm, Hanover; occasionally seen ; European.]

ORDER 15. TILIACE无. Linden FaMily.

Tilia, Tourn. Basswood. Linden. Lime-tree.

T. Americana, L. Woods; common.

[T. EuropæA, L. (European Linden.) Planted by the roadsides and in private grounds. This tree (the LiN) gave the family name to Linnæus.] 


\section{Order i6. LINACE无. Flax FAMily.}

Linum, Tourn. Flax.

L. Virginianum, L. (IVild Flax.) Dry woods.

L. Usítatissimun, L. (Cultivated Flax.) In grain fields. Plymouth, etc., Sargent. European.

Order 17. GERANIACE开. Geranium Family.

Geranium, Tourn. Cranesbill.

G. maculatum, L. (Cranesbill Geranium.) Hanover, N. H.; Norwich, Vt.; etc. Open fields.

G. Robertianum, L. (Herb Robert.) Common on moist rocks.

G. Carolinianum, L. Not uncommon; in waste places.

Erodium, L'Her. Storksbill.

E. cicutarium, L'Her. Ludlow, Vt., Miss Pollard. Rarely met with; European.

Impatiens, L. Balsam. Jewel-weed.

I. pallida, Nutt. Windsor and Hartland, Vt., etc., Leland. Less common than the next.

I. fuiva, Nutt. Common in wet places.

Oxalis, L. Wood-Sorrel.

O. Acetosella, L. (True Wood-Sorrel.) Common.

O. violacea, L. (Violet IVood-Sorrel.) Cavendish, Vt., Dr. Graves. Very unusual so far north, and probably rare.

O. corniculata, L. var. stricta, Sav. (O. stricta, L.)-(Yellow Wood-Sorrel.) A common weed and very variable.

Order i8. RUTACE无. Rue Family.

Xanthoxylum, L. Prickly Ash.

X. Americanum, Mill. Claremont, N. H., Dr. Barrows ; Norwich and Hartford, Vt., etc.

[PTelea trifoliata, L. (Hop-tree.) From the West, is often planted.]

ORder 19. ILICINE无. Holly Family.

Ilex, L. Holly.

(Formerly AQUIFOLIACE.Æ.)

I. verticillata, Gray. (Black Alder.) Common in low grounds.

Nemopanthes, Raf. (Mountain Holly.)

N. fascicularis, Raf. (N. Canadensis, DC.) Common in wet cold woods.

Order 20. CELASTRACEÆ. Staff-tree Family.

Celastrus, L. Staff-tree.

C. scandens, L. (Climbing Bitter-sweet.) Along streams.

[Euonymus atropurpureus, Jacq. (Burning Bush.) From the IVest; common in cultivation.] 
ORder 21. RHAMNACE无. BuCkThorN FAMily.

Rhamnus, Tourn. Buckthorn.

R. alnifolia, L'Her. Bear Pond, Canaan, N. H., F. B. Wallace; Norwich and Sharon, Vt., Fesup; rare.

R. Cathartica, L. Used for hedges; sometimes running wild; European.

Ceanothus, L. New Jersey Tea.

C. Americanus, L. Dry woodlands. Lebanon near Conn. river, etc.

\section{ORDER 22. VITACE巴. VINE FAMILY.}

Vitis, Tourn. Grape.

V. Labrusca, L. (Fox grape.) Often near old homesteads, sometimes cultivated; doubtful if native in this region.

V. æstivalis, Michx. (Summer grape.) Possibly native.

V. cordifolia, Michx. As the species is now understood, it may be that plants referred to this species should be included in the next.

V. riparia, Michx. (Frost grape.) Common. This is V. cordifolia, var. riparia, of Gray's Man. (IS7O). Dr. Engelmann writes ( $\left.\mathrm{I} \mathrm{S}_{7} 6\right)$ :- “ I I doubt if V. cordifolia grows at all in New England." See his synopsis of the Genus in Am. Nat., Vol. VI, p. 539. See, also, Gray's Manual (189o).

Ampelopsis, Michx. Woodbine. Virginian Creeper.

A. quinquefolia, Michx. Common on river-banks, etc.

\section{Order 23. SAPINDACE开. Soapberry Family.}

Æsculus, L. Horse-chestnut. Buckeye.

[Æ. Hippocastanum, L. (Horse-chestnut.) Common in cultivation everywhere. Native of Europe.]

[Æ. GLabra, Willd. (Fetid or Ohio Buckeye.) Not native in New England, but not rare in cultivation along with $£$. PAviA.]

Acer, Tourn. Maple.

A. Pennsylvanicum, L. (Striped Dogwood. Moose-IWood.) Woods; common.

A. spicatum, Lam. (Mountain Maple.) Common with the above.

A. saccharinum, Wang. (Sugar Maple. Rock Maple.) Yery common. var. nigrum, Torr. \& Gray. (Black Sugar Maple.) Probably not uncommon. Recognized by Michaux as a distinct species, and common outside of New England. He noticed it " at Windsor, Vt., but of inferior size." (Michaux's N. A. Sylva, Vol. I, p. I63.) It is seldom distinguished from the ordinary Sugar Maple.

A. dasycarpum, Ehrh. (White or Silver Maple.) On river-banks.

A. rubrum, L. (Red or Swamp Maple.) Very common.

[A. PLATANOIDES (Norway Maple), with fruit having long divergent wings, and A. PsEudo-Platanus with long racemes of pubescent fruit, are often planted.]

Negundo, Moench. Ash-leaved Maple. Box-Elder.

$\mathrm{N}$ aceroides, Moench. Not native in New England, but often planted and sometimes spontaneous, as on the White River at Sharon, Vt., $D r$. Baxter. 
Staphylea, L. Bladder-Nut.

S. trifolia, L. Claremont and Lebanon, N. H.; Norwich, Sargent, and IVindsor, Vt. River-banks; not very common.

\section{Order 24. ANACARDIACE无. CAShew Family.}

Rhus, L. Sumach.

R. typhina, L. (Staghorn Sumach.) Fields; very common. This species and the next two are not poisonous.

R. glabra, L. (Smooth Sumach.) Fields; common.

R. copallina, L. Claremont, N. H., Dr. Barrows; Holderness, N. H., and Fairlee, Vt., fesup. Rare.

R. venenata, DC. (Poison Sumach. Poison Dogwood.) Claremont, N. H., Dr. Barrows: Mud Pond, Canaan, N. H., Fesup; IVindsor, Vt., Leland. Not uncommon in swamps throughout the district.

R. Toxicodendron, L. (Poison Ivy.) Very common, and, like the preceding, very poisonous to the touch.

\section{ORder 25. POLYGALACE无. Milkwort Family.}

Polygala, Tourn. Milkwort.

P. paucifolia, Willd. (Fringed Polygala.) Borders of woods ; common.

P. polygama, Walt. Claremont, Dr. Barrows; New London, Miss Messer.

P. sanguinea, L. Charlestown and New London, N. H.; IVindsor, Vt.; etc.

P. verticillata, L. Claremont, Dr. Barrows. var. ambigua (P. ambigua, Nutt.) Lebanon, Sargent.

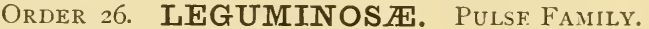

Lupinus, Tourn. Lupine.

L. perennis, L. Claremont, etc., Dr. Barrows ; Plymouth, Miss Langdon; rare; abundant farther south.

Trifolium, Tourn. Clover. Trefoil.

T. ARvense, L. (Stone Clover.) Old fields and roadsides.

T. PRATENSE, L. (Red Clover.) Meadows.

T. repens, L. (White Clover.) Everywhere common.

T. AgraRiUM, L. (Yellow or Hop-Clover.) Meadows and roadsides.

T. Procumbens, L. Claremont, Dr. Barrows; Royalton, Vt., H. Q. Ward. Uncommon; has pinnately trifoliate leaves and very small heads. This and all the preceding species are European, except T. repens.

T. Hybridum, L. (Alsike or Dutch Clover.) From Holland. Meadows.

Melilotus, Tourn. Melilot.

M. OfFicinalis, Willd. (Yellow Melilot.) Waste or cultivated grounds; both species from Europe.

M. ALBA, Lam. (White Melilot or Sweet Clover.) Near dwellings and sometimes in gardens. 
Medicago, Tourn. Medick.

M. sativa, L. (Lucerne, Alfalfa.) Claremont, N. H., Dr. Barrows ; Pomfret, Vt., A. P. Morgan. Cultivated sometimes for green fodder.

II. Lupulina, L. (Black Medick.) Claremont, Dr. Barrows. In grass ground, Hanover, etc., and often mistaken for a Trifolium.

M. MACUlata. Willd. (Spotted Medick.) Claremont, Dr. Barrows. This and the next species introduced in foreign wool, and probably transient vistors. The entire genus is European.

M. Denticulata, IVilld. Claremont, Dr. Barrozis.

Robinia, L. Locust-tree.

R. Pseudacacia, L. (Common Locust.) Naturalized from Penn. and the Southwest.

R. viscosa, Vent. (Clammy Locust.) Native of Virginia, and spreading freely.

Astragalus, Tourn. Milk-Vetch.

A. alpinus, L. On rocks at Sumner's Falls, Plainfield, N. H., Flint; very rare.

A. Robbinsii, Gray. Sumner's Falls, N. H., Flint; Hartland, Vt., Leland; very rare.

Desmodium, Desv. Tick-Trefoil.

D. nudiflorum, DC. Iry woods; common.

D. acuminatum, DC. Woods; common.

D. rotundifolium, DC. Dry rocky woods.

D. Dillenii, Darl. Open woodlands.

D. paniculatum, DC. IVoods.

D. Canadense, DC. Dry woods; common.

D. rigidum, DC. Dry hillsides.

D. Marilandicum, Boott. Copses; not very common.

Lespedeza, Michx. Bush-Clover.

L. violacea, Pers. Dry woods.

L. polystachya, Michx. (L. hirta, Ell.) Dry hillsides; common.

L. capitata, Michx. Dry hillsides ; frequent.

Vicia, Tourn. Vetch or Tare.

V. Sativa, L. (Common Vetch.) Cultivated fields; not uncommon; from Europe.

VAR. ANGUSTIFolia, Seringe. Hanover.

V. Cracca, L. Infrequent; usually in grass land.

Apios, Boerhaave. Ground-nut.

A. tuberosa, Moench. Windsor, Vt., Flint; Royalton, Vt., L. IVild; Plymouth, N. H., Sargent.

Amphicarpæa, Ell. Hog Pea-nut.

A. monoica, Nutt. Borders of woods; very common.

Cassia, Tourn. IVild Senna.

C. Marilandica, L. Hartford, Vt. Alluvial soil ; rare. 
Gleditschia, L. Honey-Locust.

G. triacanthos, L. Never truly wild in New England; common in cultivation; from the Western States, and sometimes thornless.

[Additional plants of this order :-CoronillaA raRia (Purple Coronilla), ARachis hypogea (Pea-nut), Caragana arborescens (Pea-tree), Wistaria sinensis (Chinese Wistaria), Phaseolus (Garden Bean), Pisum SATIVA (Garden Pea), besides others from the Western States and from Europe.]

Order 27. ROSACE开. Rose Family.

Prunus, Tourn. Plum. Cherry.

P. Americana, Marshall. (WVild Yellow or Red Plum.) Not common.

P. pumila, L. (Dwarf Cherry.) Islands in the Conn. river near Hanover, etc. Fesup.

P. Pennsylvanica, L. (Red Cherry.) Very common everywhere.

P. Virginiana, L. (Choke-Cherry.) Very common.

P. serotina, Ehrh. (Black Cherry.) Common.

Spiræa, L. Meadow-Sweet.

S. salicifolia, L. (Common Meadow-Sweet.) Roadsides and pastures.

S. tomentosa, L. (Hardhack. Steeple-Bush.) Roadsides; common.

S. SORBIfolia, from Siberia, with leaves like the Mountain Ash, is well established in many places.

Rubus, Tourn. Raspberry. Blackberry.

R. odoratus, L. (Purple Flowering Raspberry.) Often wrongly called Mulberry.

R. triflorus, Richardson. (Dwarf Raspberry.) Much like a Blackberry.

R. strigosus, Michx. (Wild Red Raspberry.)

R. occidentalis, L. (Black Raspberry. Thimbleberry.) Both this and all the preceding species common.

R. villosus, Ait. (High Blackberry.) Very variable in size and shape of fruit. Very common.

R. Canadensis, L. (Low Blackberry.) Dry fields. The smaller and trailing forms of the last connect with this species.

R. hispidus, L. (Running Swamp-Blackberry.) Not very common.

Dalibarda, L.

D. repens, L. Low woods; common.

Geum, L. Avens.

G. album, Gmelin. Borders of woods; common.

G. Virginianum, L. Borders of woods; common.

G. macrophyllum, Willd. Killington Mt., Vt., Rev. H. M. Denslow.

G. strictum, Ait. Moist meadows; frequent.

G. rivale, L. (Water Avens.) WVet meadows.

Waldsteinia, Willd.

W. fragarioides, Tratt. (Barren Strawberry.) Wooded hillsides; common.

Fragaria, Tourn. Strawberry.

F. Virginiana, Mill. (Field Strawberry.) Everywhere common.

F. vesca, L. (Wood Strawberry.) Less common than the preceding. 
Potentilla, L. Cinque-foil. Five-Finger.

P. arguta, Pursh. Koadsides. Hanover; Lebanon; not rare.

P. Norvegica, L. Dry fields; common.

P. argentea, L. (Silvery Cinque-foil.) Dry, barren fields; common.

P. palustris, Scop. (Marsh Five-Finger.) Cold bogs; George Pond, Enfield, etc.

P. fruticosa, L. (Shrubby Cinque-foil.) Claremont, N. H., Dr. Barrowes; Chittenden, Vt., Flint; a shrub.

P. tridentata, Ait. (Three-toothed Cinque-foil.) Kearsarge Mt., Dr. Barrows; Moosilauke Mt., Flint.

P. Anserina, L. (Silver-weed.) Claremont, N. H., Dr. Barrows ; Royalton, Vt., Ward. Near ponds and streams.

P. Canadensis, L. (Common Five-Finger.) Dry soil ; very common; stem commonly prostrate; sometimes erect.

Agrimonia, Tourn. Agrimony.

A. Eupatoria, L. (Common Agrimony.) Roadsides.

Poterium, L. Burnet.

P. Canadense, Benth \& Hook. (Canadian Burnet.) Bristol, N. H., etc., F. L. Pattee. Not common.

Rosa, Tourn. Rose.

R. blanda, Ait. (Early Rose.) Banks of Conn. river, Hanover, N. H.; Fairlee, Vt., Sargent. Not very common.

R. Carolina, L. (Swamp Rose.) Swamps and river-banks throughout.

R. humilis, Marsh. (R. lucida of most authors.)-(Dwarf Rose.) With the same range as $\mathrm{R}$. blanda, but more common. The true lucida is found on the New Hampshire seacoast.

R. nitida, Willd. (Shining Rose.) New London, Sargent; George Pond, Enfield, etc., Fesup. Rather rare.

R. RUbiginosA, L. (Sweet-brier.) Roadsides and pastures; not abundant, but widely distributed; European.

R. Cinnamomea, L. (Cinnamon Rose.) Native of Europe, very common about old dwellings, always double.

Pyrus, L. Pear. Apple.

P. arbutifolia, L. (Choke-berry.) Swamps; common, with red fruit. var. melanocarpa, Hook. With black fruit.

P. Americana, DC. (Mountain Ash.) Common.

Cratægus, L. White Thorn. Hawthorn.

[C. Oxyacantha, L. (English Hawthorn.) Cultivated for ornamental purposes, and sometimes spontaneous.]

C. coccinea, L. (Scarlet-fruited Thorn.) Common in hillside pastures, etc. var. macracantha, Dudley. Frequent and quite distinct from the type.

Perhaps a distinct species. Hanover, Lyme, Lebanon, and Enfield,

N. H.; Norwich, Hartford, etc., Vt.

C. punctata, Jacq. Widely distributed on both sides of the Conn. river.

C. Crus-galli, L. Claremont, Dr. Barrows; College Park, Hanover. Not common, and possibly not indigenous. 
Amelanchier, Medic. June-berry.

A. Canadensis, Torr. \& Gray. (var. Botryapium, T. \& G.)-(Shad-bush.) The more common form.

var. oblongifolia, Gray. Not rare; two weeks later. A smaller tree or shrub.

[This Order also includes numerous species of the Genus Prunus (Plum, Cherry, Peach, Nectarine, and Apricot); SPIR.玉A (many species); Pyrus COMMUNis (Pear); Prrus Malus (Apple); PYrUS PRUNifolia (Siberian Crab-apple); and Cydonia vulgaRIs (Common Quince).]

\section{ORdER 2S. SAXIFRAGACE开. SAXIFRAGE FAMILY.}

Saxifraga, L. Saxifrage.

S. Virginiensis, Michx. (Rock Saxifrage.) Common on rocks.

S. Pennsylvanica, L. (Swamp Saxifrage.) Common in swamps.

Tiarella, L. False Mitre-wort.

T. cordifolia, L. Common in moist ground.

Mitella, Tourn. Mitre-wort. Bishop's-Cap.

M. diphylia, L. Hillsides in rich woods; very common.

M. nuda, L. Wet woods in moss. Hanover, N. H.; Norwich, Vt.; etc. Not rare.

Chrysosplenium, Tourn. Golden Saxifrage.

C. Americanum, Schwein. Common in wet places.

Parnassia, Tourn. Grass of Parnassus.

P. Caroliniana, Michx. Queechy Gulf in Hartland, Norwich, and Royalton, Vt., L. Wild. Not common.

Philadelphus, L. Mock Orange. Syringa.

[P. GRANDIFLORUS, Willd. Cultivated from Virginia.]

[P. CORONARIUS, L. In cultivation from Europe.]

Ribes, L. Currant. Gooseberry.

R. Cynosbati, L. (Prickly Gooseberry.) Common.

R. oxyacanthoides, L. (R. hirtellum, Michx.)-(Smooth Gooseberry.) Meriden and Hanover, N. H.; Union Village and Sharon, Vt. Probably not rare in swamps.

R. lacustre, Poir. Hanover and Meriden, N. H. ; Mt. Ascutney, Vt. Cold woods and swamps.

R. prostratum, L'Her. (Fetid Currant.) Common in damp, rocky woods.

R. floridum, L'Her. (Wild Black Currant.) Woods, and frequent in old gardens.

R. rubrum, L. var. subglandulosum, Maxim. This is the native Red Currant of the White Mts. and Northern New England. The Red Currant of the garden is the European form (R. rubrum) introduced by the early settlers. The two forms however cannot readily be distinguished.

R. aureum, Pursh. (Missouri Currant.) Common in cultivation, and persisting for years about old homesteads. Native of Missouri and the West. 
Order 29. CRASSULACE无. Orpine Family.

Penthorum, Gronov. Ditch Stone-crop.

P. sedoides, L. Open wet places.

Sedum, Tourn. Stone-crop.

S. ACRE, L. (Mossy Stone-crop.) Norwich, Chelsea, and Royalton, Vt.; on rocks, etc. Frequent in gardens throughout the district.

S. Telephium, L. (Live-for-ever.) Very widely distributed and thoroughly naturalized; both species European.

\section{ORder 30. DROSERACE无. SUNDEW FAMILY.}

Drosera, L. Sundew.

D. rotundifolia, L. Peat bogs and banks of brooks; common.

D. intermedia, Hayne. var. Americana, DC. (D. longifolia. Man., 5th ed.)

Hanover, Canaan, Enfield, Plainfield, etc. Sandy shores of ponds and on decaying logs.

ORder 3i. HAMAMELIDE无. Witch-Hazel Family.

Hamamelis, L. Witch-Hazel.

H. Virginiana, L. Peculiar for blooming in October and not perfecting its fruit until the next autumn; common in woods.

ORder 32. HALORAGEÆ. Water-Milfoil Family.

Myriophyllum, Vaill. Water-Milfoil.

M. spicatum, L. Enfield Pond, Fesup; not rare.

M. verticillatum, L. Mascoma Creek, West Lebanon, etc., Fesup.

M. tenellum, Bigel. Shores of Enfield Pond, Fesup; not rare.

Proserpinaca, L. Mermaid-weed.

P. palustris, L. Swamps and ditches; common.

Hippuris, L. Mare's Tail.

[H. vulgaris, L. About ponds; rare. Near Long Pond, West Danville, Vt., Dr. Blanchard, and in Mass.; probably within our limits.]

Callitriche, L. Water-Starwort.

C. verna, L. Ponds; common.

ORder 33. LYTHRACE无. LOOSESTRIFE FAMILy.

Lythrum, L. Loosestrife.

L. Salicaria, L. (Spiked Loosestrife.) Claremont, N. H., Dr. Graves; Sharon, Vt., etc. ; often cultivated, and commonly trimorphous.

Order 34. ONAGRACE开. Evening-Primrose Family.

Ludwigia, Ell. False Loosestrife.

L. palustris, L. (Water Purslane.) Ditches; common.

Epilobium, L. Willow-herb.

E. angustifolium, L. (Often like Erechtites, called "Fire-weed.") Common in moist and newly cleared lands. 
E. lineare, Muhl. (E. palustre, var. lineare, Man., 5th ed.) Bogs; common.

E. strictum, Muhl. (E. molle, Torr.) Claremont, Dr. Barrows; not common.

E. coloratum, Muhl. IVet places; very common.

[A number of new species of this genus have recently (Man., 6th ed.) been assigned to this region, some of which doubtless are collected as forms of the above and remain to be distinguished from them.]

Enothera, L. Evening Primrose.

E. biennis, L. Fields and roadsides everywhere; many vars.; one with large flowers is found in gardens (var. grandiflora).

E. pumila, L. Dry fields; common.

Circæa, Tourn. Enchanter's Nightshade.

C. Lutetiana, L. Damp shades; common.

C. alpina, L. Rocky, wet woodlands; much like a small form of the . former.

\section{Order 35. CUCURbitACEÆ. Cucumber Family.}

Sicyos, L. Bur-Cucumber.

S. angulatus, L. IVet, shady places and waste heaps; a not uncommon weed in Hanover, etc.

Echinocystis, Torr. \& Gray. Balsam-Apple.

E. lobata, Torr. \& Gray. Banks of streams, Hanover, N. H.; Sharon, Vt.; etc.

[Cultivated species of this Order belong to the Genera:-LaGenaria (Bottle Gourd), Cucurbita (Pumpkin, Squash, and Egg Gourd), CitruLUS (IVatermelon), Cucumis (Melon and Cucumber).

\section{ORDER 36. FICOIDEÆ⿸尹}

Mollugo, L. Carpet-weed.

M. verticillata, L. A common weed in cultivated ground. Formerly included in the Pink Family (CARYOPHYLLACE E).

Order 37. UMBELLIFER Æ. Parsley Family.

Daucus, Tourn. Wild Carrot.

D. CARota, L. A noxious weed of the old world; the cultivated carrot run wild.

Angelica, L.

A. atropurpurea, L. (Archangelica atropurpurea, Hoffm.) River-banks.

Conioselinum, Fisch. Hemlock-Parsley.

C. Canadense, Torr. \& Gray. Near Goose Pond, Canaan.

Heracleum, L. Cow-Parsnip.

H. lanatum, Michx. Moist, rich ground; common.

Pastinaca, L. Wild Parsnip.

P. Sativa, L. Fields; common; from Europe. 
Ligusticum, L. Lovage.

L. Scoticum, L. Near dwellings; Hanover, etc.; perhaps a garden escape.

Cryptotænia, DC. Honewort.

C. Canadensis, DC. Thickets; not rare.

Sium, Tourn. Water-Parsnip.

S. cicutæfolium, Gmelin. (S. lineare, Michx.) Wet places; common.

Zizia, Koch. Neadow-Parsnip.

Z. aurea, Koch. (Thaspium aureum, var. apterum, Gray's Man., 5th. ed.) In moist meadows; common.

Carum, L. Caraway.

C. CARUI, L. Everywhere near dwellings; European.

Cicuta, L. Water-Hemlock.

C. maculata, L. (Spotted Cowbane.) Root very poisonous; common.

C bulbifera, L. Swamps; seldom fruiting, the flowers replaced by bulblets.

Conium, L. Poison Hemlock.

C. maculatum, L. On waste heaps; a virulent poison. Hanover, etc. From Europe.

Osmorrhiza, Raf. Sweet Cicely.

$O$. brevistylis, DC. Rich, moist woods.

O. longistylis, DC. With, but less common than, the preceding.

Hydrocotyle, Tourn. Water Pennywort.

H. Americana, L. Wet places; common.

Sanicula, Tourn. Sanicle. Black Snakeroot.

S. Marilandica, L. With the variety but more common. Borders of woods.

var. Canadensis, Torr. (S. Canadensis, L.)

[This large Order also includes,-APIUM GRAVEOLEns (Celery), CARUM Petroselinum (Parsley), Feniculumi vulgare (Fennel), etc.]

Order 38. ARALIACE无. Ginseng Family.

Aralia, Tourn.

A. racemosa, L. (Spikenard.) Rich woodlands; often cultivated.

A. hispida, Vent. (Bristly Sarsaparilla.) Rocky places; frequent.

A. nudicaulis, L. (Wild Sarsaparilla.) Borders of woods ; common.

A. quinquefolia, Dec. \& Pla. (Ginseng.) Rich woods; not infrequent.

A. trifolia, Dec. \& Pla. (Dwarf Ginseng. Ground-nut.) Very common in woods.

ORDER 39. CORNACE巴. DOGWOOD FAMILY.

Cornus, Tourn. I)ogwood. Cornel.

C. Canadensis, L. (Bunch-berry.) Common.

C. florida, L. (Flowering Dogwood.) Plainfield, N. H., Miss Sterens; Springfield, Vt., Rev. S. Hayzuard; Charlestown and New London, N. H.; Pomfret, Vt., etc.; rare so far northward. 
C. circinata, L'Her. (Round-leaved Cornel.) Hanover, N. H.; West Hartford, Vt., Flint; and Killington Mt., Vt., Rev. H. M. Denslow.

C. sericea, L. (Silky Cornel.) Borders of ponds ; frequent.

C. stolonifera, Michx. (Red-osier Dogwood.) Wet places; common.

C. paniculata, L'Her. (Panicled Cornel.) Charlestown, Dr. Graz'es; Plymouth, Miss Langdon; rare.

C. alternifolia, L. (Alternate-leaved Cornel.) Common by the roadside everywhere.

Nyssa, L. Tupelo. Pepperidge. Sour-Gum Tree.

N. sylvatica, Marsh. (N. multiflora, Wang.) On the outlet of Squam Lake, N. H. ; not common. [Not rare in Boscawen, Barton.] 


\section{Division II. GAMOPETALOUS EXOGENS.}

Order 40. CAPRIFOLIACE开. Honeysuckle Family.

Sambucus, Tourn. Elder.

S. Canadensis, L. (Black-berried Elder.) Common.

S. racemosa, L. (S. pubens, Michx.)-(Red-berried EIder.) Common.

Viburnum, L. Arrow-wood.

V. lantanoides, Michx. (Hobble-bush.) Frequent in woods.

V. Opulus, L. (High Cranberry-bush.) Hanover, Lebanon, and New London, N. H. ; Sharon, etc., Vt.: low ground, not rare. The Snow Ball Tree from Europe is a cultivated form of this species.

V. pauciflorum, Pylaie. Claremont, Dr. Barrows; belongs farther northward, and doubtless rare.

V. acerifolium, L. (Maple-leaved Arrow-wood.) Rocky woods ; common.

V. dentatum, L. (Arrow-wood.) Wet places.

V. cassinoides, L. (V. nudum. var. cassinoides, Torr. \& Gray.) - (Witherod.) Swamps in Lebanon and Hanover, N. H.; Sharon and Hartford, Vt. ; etc. ; common.

V. Lentago, L. (Sheep-berry.) Wet river-banks, etc.; not rare.

Triosteum, L. Fever-wort. Horse-Gentian.

T. perfoliatum, L. White River Junction and Norwich, Vt., etc. Rich woodlands.

Linnæa, Gronov. Twin-flower.

L. borealis, Gronov. Common in bogs and mossy woods. The favorite flower of the great Linnæus, and named for him.

Symphoricarpos, Dill. Snowberry.

[S. racemosus, Michx. Common in cultivation; native of western Vt. S. VULG.ARIS and S. OCCIDENTALIS from the Western States are sometimes in private grounds.]

Lonicera, L. Honeysuckle. Woodbine.

L. ciliata, Muhl. (Fly-Honeysuckle.) Rocky woods; common.

L. cærulea, L. (Mountain Fly-Honeysuckle.) Claremont, Dr. Barrows; Bear Pond, Canaan, E. S. Comings; Enfield Pond, A. C. French.

L. glauca, Hill. (L. parviflora of Man., 5th ed.) (Twining Honeysuckle.) Hanover, N. H.; Norwich, Vt. ; etc ; not rare.

[L. TATARICA, L. (Tartarian Honeysuckle.) The common Bush Honeysuckle of cultivation ; from Europe.]

Diervilla, Tourn.

D. trifida. Moench. (Bush Honeysuckle.) Common in fence rows.

Order 4I. RUBIACE正. Madder Family.

Houstonia, L.

H. cærulea, L. (Bluets. Innocence.) Pastures; commonly dimorphous. 
Cephalanthus, L. Button-bush.

C. occidentalis, L.' New Found Lake, Dr. Barrowes; Goose Pond, Canaan ; Andover, A.C. French ; Plymouth, Sargent; etc.

Mitchella, L. Partridge-berry.

M. repens, L. Dry woods; common under trees and dimorphous.

Galium, L. Bedstraw. Cleavers.

G. Mollugo, L. North Pomfret, Vt., Morgan.

G. Aparine, L. (Cleavers. Goose-Grass.) Moist grounds, Norwich, Vt., Eggleston: not common.

G. Kamtschaticum, Steller, (G. circæzans, var. montanum, Torr. \& Gray.) Killington Peak, Vt., Denslow.

G. circæzans, Michx. (Wild Liquorice.) Woods; not rare.

G. lanceolatum, Torr. (Wild Liquorice.) Pomfret, Vt., Morgan; Hanover, French,-also Weirs, N. H.; infrequent in dry woods.

G. trifidum, L. (Small Bedstraw.) Swamps; very variable; common.

G. asprellum, Michx. (Rough Bedstraw.) Low thickets; abundant.

G. triflorum, Michx. (Sweet-scented Bedstraw.) Rich woodlands; common.

Order 42. COMPOSIT仺. Composite Family.

Eupatorium, Tourn. Thoroughwort. Boneset.

E. purpureum, L. (Purple Thoroughwort.) Common in swamps.

E. perfoliatum, L. (Medicinal Thoroughwort.) Low grounds; common.

E. ageratoides, I.. (White Snake-root.) Rich woods.

Solidago, L. Golden-rod.

S. squarrosa, Muhl. Claremont, N. H., Graves; Windsor, Flint; Killington Mt., Eggleston, and Fairlee Pond, Vt., Fesup.

S. cæsia, L. Borders of woodlands; common.

S. latifolia, L. Shaded banks; common.

S. bicolor, L. Dry banks. Also var. concolor, Torr. \& Gray, yellow rays.

S. macrophylla, Pursh. (S. thyrsoidea, E. Meyer.) Claremont, N. H., Dr. Barrows; Mt. Ascutney, Leland,-and Killington Mt., Vt., Eggleston.

S. Virgaurea, L., var. alpina, Bigel. Moosilauke Mt., Flint; Killington Peak, Vt., Dr. Robbins.

S. humilis, Pursh. (S. Virgaurea, var. humilis, Gray.) Mountains.

S. puberula, Nutt. Killington, Mt., Vt., Denslow ; Rochester, Vt., Eggleston.

S. uliginosa, Nutt. (S. stricta, Ait.) Peat bogs. Canaan and Hanover, N. H. ; Sharon, Dr. Baxter, and Norwich, Vt., Fesup; occasional.

S. rugosa, Mill. (S. altissima, Man., 5th ed.) Fields; common.

S. neglecta, Torr. \& Gray. Swamps, and very variable. Some forms as determined by Dr. Gray are much like "uliginosa" and "arguta." Perhaps not very rare; Canaan, Fesup.

S. arguta, Ait. (S. Muhlenbergii, Torr. \& Gray.) Borders of woods; frequent.

S. juncea, Ait. (S. arguta, Torr. \& Gray.) Borders of woodlands; common. 
S. serotina, Ait. (S. gigantea, Man., 5th ed.) Roadsides; common. var. gigantea, Gray. (S. serotina, Man., 5th ed.) low ground; common.

S. Canadensis, L. Fields and roadsides; very common and variable.

S. nemoralis, Ait. Dry, sterile fields; very common.

S. lanceolata, L. Roadsides, etc. ; very common.

Sericocarpus, Nees. White-topped Aster.

S. conyzoides, Nees. Dry grounds.

Aster, L. Starwort. Aster.

A. corymbosus, Ait. Woodlands ; common.

A. macrophyllus, L. Borders of woods; common.

A. Novæ-Angliæ, L. Royalton, Vt., Ward; more common southward.

A. patens, Ait. Dry ground.

A. undulatus, L. Dry woods; common.

A. cordifolius, L. Woodlands; very common.

[A. Lindleyanus, T. \& G. has been found at Lisbon, N. H., by $C$. $E$. Faxon, just beyond our limits.]

A. lævis, L. Woodlands.

A. ericoides, L. Dr. Barrows. More common southward.

A. polyphyllus, Willd. "Heads larger and flowering earlier than the preceding."

A. multiflorus, Ait. Claremont, N. H., Dr. Graves; Norwich, Vt., Sargent.

A. dumosus, L. Claremont, Dr. Barrowes ; scarce.

A. vimineus, Lam. (A. Tradescanti, Man., $5^{\text {th }}$ ed.) Moist banks and variable.

A. diffusus, Ait. (A. miser, Man., 5th ed.) Roadsides and fields; very common and variable.

A. Tradescanti, L. (A. tenuifolius, Man., $5^{\text {th }}$ ed.) With the next, but not readily distinguished from it.

A. paniculatus, Lam. (A. simplex, Man., 5th ed.) Moist banks; very common.

A. salicifolius, Ait. (A. carneus, Man., 5th ed.) What appears to be this species has been found with the preceding.

A. Novi-Belgii, L. (A. longifolius. Man., $5^{\text {th }}$ ed.) Moist ground and very variable.

A. puniceus, L. Swamps ; very common and variable

A. umbellatus, Mill. (Diplopappus umbellatus, Torr. \& Gray.) Moist thickets; common.

A. linariifolius, L. (Diplopappus linariifolius, Houk.) Dry grounds; not very common.

A. acuminatus, Michx. Rich woods; frequent.

A. nemoralis, Ait. George Pond, Enfield. [Todd Pond, Bradford, Barton.]

Erigeron, L. Fleabane.

E. Canadensis, L. (Horseweed.)

E. annuus, Pers. (Daisy Fleabane.) Fields and waste places.

E. strigosus, Muhl. (Daisy Fleabane.) A coarser plant than the rest. This and both the above common weeds. 
E. hyssopifolius, Michx. (Aster graminifolius, Pursh.) On rocks in Quechee Gulf, Vt., Fesup : quite rare.

E. bellidifolius, Muhl. (Daisy-leaved Fleabane. Robin's Plantain.) Moist meadows and banks; very common.

E. Philadelphicus, L. Moist ground; common.

Antennaria, Gaertn. Plantain-leaved Everlasting.

A. plantaginifolia, Hook. Sterile banks ; very common.

Anaphalis, DC. Pearly Everlasting.

A. margaritacea, Benth. \& Hook. Dry fields; very common.

Gnaphalium, L. Cudweed.

G. polycephalum, Michx. (Common Everlasting.)

- G. decurrens, Ives. (Everlasting.) Old fields.

G. uliginosum, L. (Low Cudweed.) Roadsides. All the species common.

Inula, L. Elecampane.

I. Helenium, L. A coarse plant on roadsides and in pastures. Native of Europe.

Ambrosia, Tourn. Ragweed.

A. trifida, L. (Great Ragweed.) Grantham, Burton.

A. artemisizfolia, L. (Roman Wormwood.) A common farm weed.

Xanthium, Tourn. Cocklebur. Clotbur.

X. Spinosu M, L. On waste heaps. From Tropical America.

X. strumarium, L. White River Junction, Vt., Flint. Barnyards, etc. ; not commom. From India.

Heliopsis, Pers. Ox-eye.

H. scabra, Dunal. (H. lævis, var. scabra, T. \& G.) Norwich, Vt., Sargent. A chance immigrant from the west.

Rudbeckia, L. Cone-flower.

R. laciniata, L. Low grounds.

R. hirta, L. Introduced into meadows from the Western States.

Helianthus, L. Sunflower.

H. giganteus, L. Randolph, Vt., Bates; Royalton, Vt., Ward.

H. annuus, L. (Common Sunflower.) Long cultivated and often spontaneous.

I1. strumosus, L. Charlestown, Dr. Graves; common farther south.

H. divaricatus, L. Not common.

H. decapetalus, L. Banks of streams, etc.; very common.

H. tuberosus, L. (H. doronicoides, Man., 5th ed.)-(Jerusalem Artichoke.) Often found in old gardens. Dr. Gray has shown that both this species and $\mathrm{H}$. annuus originated in America.

Bidens, L. Bur-Marigold.

B. frondosa, L. (Common Beggar-ticks.) The entire genus is found in swamps and wet places.

B. connata, Muhl. (Swamp Beggar-ticks.) Common in ditches, etc.

B. cernua, L. (Smaller Bur-Marigold.) Not well distinguished from the next; both probably common. 
B. chrysanthemoides, Michx. (Larger Bur-Marigold.)

B. Beckii, Torr. (IVater Marigold.) Claremont, N. H., Dr. Barrows; Fairlee Pond, Vt., Fesup. Rare.

Galinsoga, Ruiz \& Pavon.

G. PARviflora, Cav. From South America. On waste heaps; IVindsor, Vt., Leland.

Anthemis, L. Chamomile.

A. Cotula, DC. (Maruta Cotula, DC.)-(May-weed.) From Europe; common by roadsides.

A. ARvensis, L. (Corn Chamomile.) From Europe. Occasionally introduced with grass seed.

[A. NobILIs, L. (Garden Chamomile.) In old gardens.]

Achillea, L. Yarrow.

A. Millefolium, L. Fields; common.

[A. PтAкmica, L. (Sneezewort.) In old gardens; both single and double.]

Chrysanthemum, Tourn. Ox-eye I)aisy.

C. Leucanthemum, L. (Leucanthemum vulgare, Lam.)-(Ox-eye or White Daisy. White-weed.) From Europe. Everywhere in grass ground.

[C. Parthenium, Pers. (Feverfew.) In old gardens.]

Tanacetum, L. Tansy.

T. vUlgare, L. Roadsides everywhere; European weed.

Artemisia, L. Wormwood.

A. vulgaris, L. (Mugwort.) Near dwellings; from Europe.

A. Absinthiun, L. (IVormwood.) Escaped from cultivation; Enfield, etc. ; from Europe.

Tussilago, Tourn. Coltsfoot.

T. FARFARA, L. Clayey soil, along brooks. Thought to have come from Europe, but now found far from cultivation.

Petasites, Tourn. Sweet Coltsfoot.

[P. palmata, Gray. (Nardosmia palmata, Hook.) A rare plant reported on both our northern and southern borders, and probably within our limits.]

Senecio, Tourn. Groundsel.

S. VULGARIS, L. In cultivated grounds; not common; from Europe.

S. aureus, L. (Golden Ragwort.) Several varieties; in both wet and dry ground.

Erechtites, Raf. Fireweed.

E. hieracifolia, Raf. Clearings that have been burned over. This and Epilobium augustifolium are the most common Fireweeds.

Arctium, L. Burdock.

A. LAPPA, L. (Lappa officinalis, All.) Roadsides; very common and variable; from Europe. 
Cnicus, Tourn. Thistle.

C. Lanceolatus, Hoffm. (Cirsium, Scop.)-(Common Thistle.) From Europe. In pastures.

C. altissimus, Willd., var. discolor, Gray. (Cirsium discolor, Spreng.) Fields, etc.

C. muticus, Pursh. (Cirsium, Michx.)-(Swamp Thistle.) Common.

C. pumilus, Torr. (Cirsium, Spreng.)-(Pasture Thistle.) Fields; common.

C. ARvensis, Hoffm. (Cirsium, Scop.)-(Canada Thistle.) Everywhere. Introduced from Europe, by way of Canada, during the Revolutionary war.

Krigia, Schreber. Dwarf Dandelion.

K. Virginica, Willd. Hanover, N. H. ; Olcott, Vt., etc.

Cichorium, Tourn. Chicory or Succory.

C. Intrius, L. Fields and roadsides. Hanover, etc. From Europe.

Tragopogon, L. Goat's-beard.

T. PORRIfolius, L. (Salsify. Oyster-plant.) New London, Sargent; Hanover, etc. A garden escape.

Hieracium, Tourn. Hawkweed.

H. aurantiacum, L. From Europe. Found several times by the roadside ; Norwich, Vt.; Royalton, Vt., Ward.

H. Canadense, Michx. Dry woods; frequent.

H. paniculatum, L. Open woods; not uncommon.

H. venosum, L. (Rattlesnake-weed.) Woods and plains; common.

H. scabrum, Michx. Dry woods; very common.

H. Gronovii, L. Not common. Dr. Barrows.

Prenanthes, Vaill. Rattlesnake-root.

P. alba, L. (Nabalus, Hook.)-(White Lettuce.) Borders of rich woods.

P. serpentaria, Pursh. (Nabalus Fraseri, DC.)-(Lion's-foot.) Borders of woods ; not rare.

P. altissima, L. (Nabalus, Hook.) Rich, moist woods.

Taraxacum, Haller. Dandelion.

T. OfFicinale, Weber. (T. Dens-leonis, Desf.) Fields and pastures; introduced from Europe.

Lactuca, Tourn. Wild Lettuce.

L. Canadensis, L. Borders of fields; common.

L. integrifolia, Bigel. (L. Canadensis, var. integrifolia, Torr. \& Gray.) With the above.

L. hirsuta, Muhl. (L. Canadensis, var. sanguinea, Torr. \& Gray.) Not uncommon.

L. leucophæa, Gray. (Mulgedium, DC.)-(Blue Lettuce.) Low grounds; common.

Sonchus, L. Sow-Thistle.

S. oleraceus, L. Waste heaps; common.

S. ASPER, Vill. North Pomfret, Vt., A. P. Morgan. Both European weeds. 
ORDER 43. LOBELIACE五. LOBELIA FAMILY.

\section{Lobelia, L.}

L. cardinalis, L. (Cardinal-flower.) Borders of streams and ponds in Lebanon, Canaan, New London, IHanover, etc., N. H.; and also in Vermont. Sometimes rose-color or nearly white.

L. spicata, Lam. In gravelly soil.

L. Kalmii, L. Near ponds and streams. Pomfret. Morgan; Sharon, Dr. Baxter; Chelsea and Olcott, Vt.; Olcott Falls, N. H., Sargent.

L. inflata, L. (Indian Tobacco.) Fields; very common.

L. Dortmanna, L. (Water Lobelia.) In ponds; New London, Sargent ; Canaan, Plainfield, etc. Not very rare.

\section{Order 44. CAmpanUlace无. Campanula Family.}

Specularia, Heister. Venus's Looking-glass.

S. perfoliata, A. DC. Sterile grounds.

Campanula, Tourn. Bellflower.

C. RAPUNCUloides, L. Escaped from gardens; a European species.

C. rotundifolia, L. (Harebell.) Rocky or sandy banks; common.

C. aparinoides, Pursh. (Marsh Bellflower.) Wet meadows; in the grass.

ORder 45. ERICACE乍. Heath Family.

Gaylussacia, HBK.

G. resinosa, Turr. \& Gray. (Huckleberry.) Swamps; Hanover and Lebanon, N. H.; Hartford, Vt.; not abundant.

Vaccinium, L. Blueberry. Bilberry. Cranberry.

V. Pennsylvanicum, Lam. (Low Blueberry.) Very common and ripe early.

V. Canadense, Kalm. (Canada Blueberry.) Swamps and low grounds; common.

V. vacillans, Solander. (Half-high Blueberry.) Hanover, N. H.; Hartford and Fairlee, Vt.; N. Pomfret, Vt., Morgan. Not common; intermediate in size between the preceding and the next.

V. corymbosum, L. (High or Swamp Blueberry.) Kipens later ; abundant and variable.

V. uliginosum, L. (Bog Bilberry.) Moosilauke Mt., Flint.

V. Vitis-Idæa, L. (Cowberry. Mountain Cranberry.) Moosilauke Mit., Flint; Cardigan Mt., A. Fairbanks.

V. Oxycoccus, L. (Small Cranberry.) Bogs; frequent.

V. macrocarpon, Ait. (Common Cranberry.) Bogs; common.

Chiogenes, Salisb. Creeping Snowberry.

C. serpyllifolia, Salisb. (C. hispidula, Torr. \& Gray.) Bogs and mountain woods: abundant.

Arctostaphylos, Adans. Bearberry.

A. Uva-ursi, Spreng. Fairlee Cliffs, Vt., F.O. Grover; and not rare both north and south of our limits. 
Epigæa, L. Trailing Arbutus. May-flower of New England.

E. repens, L. Woods and clearings; abundant.

Gaultheria, Kalm. Wintergreen or Checkerberry.

G. procumbens, L. WVoods; very common.

Andromeda, L.

A. polifolia, L. Peat bogs. Lebanon, Meriden, Canaan, etc.

A. ligustrina, Muhl. Wet grounds; Enfield, F. G. Atruell; Sunapee Lake, B. P. Ruggoles.

Cassandra, Don. Leather-Leaf.

C. calyculata, Don. Swamps; common.

Kalmia, L. Laurel.

K. angustifolia, L. (Sheep Laurel.) Common.

K. glauca, Ait. (Pale Laurel.) Cold bogs. Lebanon, Meriden, Hanover, etc

K. latifolia, L. (Mountain Laurel. Spoon-wood.) Ashland, on Squam Lake, Sargent; more common southward.

Rhododendron, L. Rose Bay. Azalea. Rhodora.

R. nudiflorum, Torr. (Azalea nudiflora, L.)—(Purple Azalea.) Swamps;

Lebanon and New London, N. H.; Norwich, Windsor, Ludlow, and. Hartford, Vt. ; not abundant.

R. viscosum, Torr. (Azalea viscosa, L.)-(White Azalea.) Sunapee Lake, B. P. Ruggles. Not common.

R. Rhodora. Don. (Rhodora Canadensis, L.) Peat swamps. Meriden, Dr. Barrows; Hanover, Fesup: Canaan, Wallace: Plymouth, Sargent.

R. maximum, L. (Great Laurel.) This superb plant has been found in North Grantham, C. H. Hitchcock, Dr. Barrows, G. C. Barton.

Ledum, L. Labrador 'Tea.

L. latifolium, Ait. Cold bogs and mountain woods. Lebanon, E. $A$. Mack; Bear Pond in Canaan, Wallace: Cardigan Mt., A. Fairbanks; New London, Carter ; and probably not infrequent.

Loiseleuria, Desv. Alpine Azalea.

L. procumbens, Desv. Moosilauke Mt., A. Fairbanks.

Chimaphila, Pursh. Pipsissewa.

C. umbellata, Nutt. (Prince's Pine.) Dry woods.

C. maculata, Pursh. With the above.

Moneses, Salisb. One-flowered Pyrola.

M. grandiflora, Salisb. (M. uniflora, Gray.) Woods. Lebanon and Hanover, N. H.; Norwich and Royalton, Vt.

Pyrola, Tourn. Wintergreen. Shin-Leaf.

P. secunda, L. All the species common in rich woods.

P. chlorantha, Swartz. Woods.

P. elliptica, Nutt. (Shin-Leaf.) Woods.

P. rotundifolia, L. Woods; several varieties.

Pterospora, Nutt. Pine-drops.

P. Andromedea, Nutt. "Parasitic apparently on the roots of pines;" very rare. Lebanon, near Olcott Falls, Fesup. 
Monotropa, L.

M. uniflora, L. (Indian Pipe.) Dark woods; common.

M. Hypopitys, L. (Pine-sap.) Under pine trees. North Pomfret, Vt., Morgan ; Royalton, Vt., Ward.

\section{Order 46. PRIMULACE出. Primrose Family.}

\section{Trientalis, L.}

T. Americana, Pursh. (Star-flower.) IVet woods.

Steironema, Raf.

S. ciliatum, Raf. (Lysimachia ciliata, L.) Low grounds; common.

S. lanceolatum, var. hybridum, Gray. (Lysimachia lanceolata, Walt.)

Banks of Conn. river.

Lysimachia, Tourn. Loosestrife.

L. quadrifolia, L. (Four-leaved Loosestrife.) IVoods; common.

L. stricta, Ait. (Strict Loosestrife.) Low grounds; common.

L. thyrsiflora, L. (Tufted Loosestrife.) Mud Pond, Plainfield, N. H., Dr. Barrozes; Royalton, Vt., Ward.

L. NUMMUlaria, L. (Moneywort.) A troublesome weed in dooryards;: European.

Anagallis, Tourn. Pimpernel.

A. ARvensis, L. An occasional weed in gardens; European.

Order 47. OLEACEÆ. Olive Family.

Fraxinus, Tourn. Ash.

F. Americana, L. (White Ash.) Common in rich woods.

F. pubescens, Lam. (Red Ash.) Squam Lake, N. H.; Randolph, Vt., Bates; occasionally seen.

F. sambucifolia, Lam. (Black or Brown Ash.) Swamps; common.

Ligustrum, Tourn. Privet.

L. vulgare, L. Naturalized in places. Dr. Barrozes. From Europe; used for hedges.

[Chionanthus Virginica (The Fringe-tree of the South), Syringa vulgaris (Common Lilac), and S. Persica (Persian Lilac), are in common cultivation.]

\section{ORdER 48. APOCYNACE开. DOgBANE FAMILy.}

Apocynum, Tourn.

A. androsæmifolium, L. (Dogbane.) Borders of thickets; common.

A. cannabinum, L. (Indian Hemp.) River-banks; frequent.

\section{Order 49. ASCLEPIADACE无. Milkweed Fanily.}

Asclepias, L. Milkweed. Silkweed.

A. tuberosa, L. (Butterfly-weed.) Dry fields.

A. purpurascens, L. (Purple Milkweed.) Charlestown, N. H., Dr. Graves; Royalton, Vt., Ward. 
A. incarnata, I. (Swamp Milkweed.) Swamps; common. var. pulchra, Pers. With the type.

A. Cornuti, Decaisne. (Common Milkweed.) Everywhere.

A. obtusifolia, Míchx. Roadsides; not common.

A. phytolaccoides, Pursh. (Poke Milkweed.) Common.

A. quadrifolia, L. Woods; not rare.

A. verticillata, L. Geological Survey of $\Lambda$. H. ; probably rare.

Vincetoxicum, Moench.

V. NIGRUM, Moench. Windsor, Vt., Leland; rare, introduced from Europe.

\section{Order 5o. GENTIANACE开. Gentian Family.}

Gentiana, Tourn. Gentian.

G. crinita, Froel. (Fringed Gentian.) Moist meadows. Hanover Centre, Lyme, etc.; not abundant.

G. quinqueflora, Lam. (Five-flowered Gentian.) Hillside woods and fields. Hanover, Lebanon, etc., Fesup; not very common.

G. Andrewsii, Griseb. (Closed Gentian.) Wet ground; common.

Menyanthes, Tourn. Buckbean.

M. trifoliata, L. In ponds and bogs. Hanover and Meriden, N. H. ; Norwich, Vt. ; etc.

Limnanthemum, Gmelin. Floating-Heart.

L. lacunosum, Griseb. In ponds; common.

Order 5i. POLEMONIACE开. Polemonium Family.

Phlox, L. Phlox.

[P. Paniculata, P. Reptans and P. subulata (Moss Pink), perennials; also P. DRUMMONDII, an annual;- -all from the West and South; havelong been cultivated in old gardens.]

Polemonium, Tourn. Greek Valerian.

[P. CARULEUM (Jacob's Ladder) of the Middle States, a rare native of New Hampshire, is often cultivated; as also is P. REPTANS (Greek Valerian) from the same region.]

ORDER 52. HYDROPHYLLACE无. WATERLEAF FAMily.

Hydrophyllum, Tourn. Waterleaf.

H. Virginicum, L. (Common Waterleaf.) Damp woods.

Order 53. BORRAGINACE无. Borage Family.

Cynoglossum, Tourn. Hound's-Tongue.

C. officinale, L. (Common Hound's-Tongue.) Pastures and roadsides; from Europe.

C. Virginicum, L. (Wild Comfrey.) Olcott Falls, etc.

Echinospermum, Lehm. Stickseed.

E. Virginicum, Lehm. (Cynoglossum Morisoni, DC.)-(Beggar's Lice.)

Borders of woods.

E. LApPula, Lehm. Waste heaps; European weed. 
Myosotis, Dill. Forget-me-not.

M. laxa, Lehm. (M. palustris, var. laxa, Gray.) Hanover, N. H. ; Sharon, Vt., Dr. Baxter; Randolph, Vt., Rev. F. A. Bates.

M. arvensis, Hoffm. Sharon, Vt., Dr. Baxter; Hanover, N. H. ; a garden weed.

M. verna, Nutt. Windsor, Vt., Leland; not common.

[M. PAlustris. IVithering. This species, the true Forget-me-not of Europe, is cultivated, and possibly spontaneous.]

Lithospermum, Tourn. Gromwell.

L. Arvense, L. West Lebanon, Dr. E. E. Phelps; not often seen.

L. OFFICINALE, L. Roadsides and pastures; both species European. weeds.

Symphytum, Tourn. Comfrey.

S. OfFicinale, I. A not common garden escape; from Europe.

Echium, Tourn. Bugloss.

E. vulgare, L. (Blue Thistle.) Hanover, N. H.; Pomfret, Sharon, and. Royalton, Vt. Roadsides and fields; European.

Mertensia, Roth. Lungwort.

[M. VirginicA, DC. (Virginian Cowslip. Blue Bells.) A native of the West and often cultivated.]

Order 54. CONVOLVULACE无. Convolvulus Family.

Ipomœa, L. Morning-Glory.

[I. Quanoclit (Cypress-Vine), I. Coccinea (Scarlet Morning-Glory), and I. PURPUREA (Common Morning-Glory) are everywhere in gardens.]

Convolvulus, Tourn. Bindweed.

C. spithamæus, L. (Calystegia spithamæa, Pursh.) Dry fields; Hanover, N. H., Fesup; Norwich, Vt., E. D. Kelley.

C. sepium, L. (Calystegia sepium, R. Br.)-(Hedge Bindweed.) Banks of streams and variable.

var. Americanus, Sims, is glabrous and common.

var. repens, Gray, is pubescent.

C. ARVEnsis, L. A smaller procumbent plant and common in old fields.

[A double flowering species, sold by the florists as "CALYsTEGIA PUBEsCENs from China," when once planted spreads rapidly.]

Cuscuta, Tourn. Dodder.

C. Gronovii, Willd. Low grounds; parasitic on herbs, etc. ; not rare.

Order 55. SOLANACE无. Nightshade Family.

Solanum, Tourn. Nightshade.

S. Dulcamara, L. (Bittersweet.) Naturalized from Europe.

S. nigrum, L. (Common Nightshade.) Shaded grounds.

Physalis, L. Ground Cherry.

P. Virginiana, Mill. (P. viscosa, Gray's Man., 5th ed.) Sandy soils.

P. pubescens, L. An occasional weed; neither species common. 
Nicandra, Adans. Apple of Peru.

N. Physaloides, Gaertn. In waste ground, but not common. Native of Peru.

Lycium, L. Matrimony-Vine.

L. VUlgare, I) unal. Near dwellings where it was once cultivated; European.

Hyoscyamus, Tourn. Henbane.

H. NIGER, L. Rarely seen; roadsides, etc. From Europe.

Datura, L. Jamestown-weed. Jimson-weed.

D. Stramonium, L. (Stramonium. Thorn Apple.) Waste heaps. From Asia.

D. Tatula, L. (Purple Thom Apple.) Waste heaps. From tropical America.

[Cultivated species of this Order are:-LyCopersicum ESCULENTUM (Tomato), Solanum tuberosum (Common Potato), and Nicotiana TABACUM (Tobacco).]

ORder 56. SCROPHULARIACE开. Figwort FAMILY.

Verbascum, L. Mullein.

V. Thapsus, L. (Common Mullein.) Fields everywhere.

V. Blattaria, L. (Moth Mullein.) Less common, both species European weeds.

Linaria, Tourn. Toad-Flax.

L. Canadensis, Dumont. Sandy soil; cummon.

L. vUlgaris, L. (Toad-Flax. Butter and Eggs.) Roadsides; European.

Scrophularia, Tourn. Figwort.

S. nodosa, L., var. Marilandica, Gray. Roadsides; Lebanon, N. H., Fesup; Royalton, Vt., Ward.

Chelone, Tourn. Turtle-head. Snake-head.

C. glabra, L. Wet places; common.

Pentstemon, Mitchell. Beard-tongue.

P. pubescens, Solander. Windsor, Vt., Leland; Norwich, Vt., G. A.

- Loveland. Not common.

P. lævigatus, Solander, var. Digitalis, Gray. (P. Digitalis, Nutt.) Lebanon, perhaps introduced with grass-seed.

Mimulus, L. Monkey-flower.

M. ringens, L. Wet places; common.

Gratiola, L. Hedge-Hyssop.

G. Virginiana, L. Wet places.

G. aurea, Muhl. Margins of ponds. Meriden, Dr. Barrows.

Ilysanthes, Raf. False Pimpernel.

I. riparia, Raf. (I. gratioloides, Benth.) Sandy borders of ponds and streams. 
Veronica, L. Speedwell.

V. Virginica, L. (Culver's-root.) Roadsides in rich soil; not common, appearing like a garden escape.

V. Anagallis, L. (Water Speedwểi.) Ditches.

V. Americana, Schwein. (American Brooklime.) Brooks and ditches.

V. scutellata, L. (Marsh Speedwell.) Bogs.

V. officinalis, L. (Common Speedwell.) Claremont, Dr. Barrozes.

V. serpyllifolia, L. (Thyme-leaved Speedwell.) With grass; common.

V. peregrina, L. (Purslane Speedwell.) A weed in cultivated grounds.

V. ARvensis, L. (Corn Speedwell.) Claremont, Dr. Barrozes.

V. Buxbaumin, Tenore. Hanover; a weed in gardens and lawns.

Gerardia, L. False Foxglove.

G. pedicularia, L. Dry woods. Fairlee Cliffs, Vt., etc.

G. flava, L. (Downy False Foxglove.) Dry woods.

G. quercifolia, Pursh. (Oak-leaved Foxglove.) Wooded hillsides. Fairlee Cliffs, Vt., etc.

G. tenuifolia, Vahl. (Slender Foxglove.) Dry woods. Dr. Barrows.

Pedicularis, Tourn. Lousewort.

P. Canadensis, I. Hanover, etc. IVoods and banks.

Melampyrum, Tourn. Cow-Wheat.

M. Americanum, Michx. IVoods. Common.

\section{ORDER 57. OROBANCHACE王. BROOM-RAPE FAMILY.}

Epiphegus, Nutt. Beech-drops.

E. Virginiana, Bart. Usually under Beech trees. Hanover and New London, N. H.; Norwich and Royalton, Vt., not rare.

Conopholis, Wallroth. Cancer-root.

C. Americana, Wallroth. In old woods. Claremont, N. H., Dr. Graves; [West Rutland, Vt., Denslow, beyond our limits.]

Aphyllon, Mitchell. Naked Broom-rape.

A. uniflorum, Gray. Borders of woods; not rare.

ORdER 58. LENTIBULARIACE无. BLADDERWORT FAMILY.

Utricularia, L. Bladderwort.

U. inflata, Walt. Claremont, Dr. Graves; ponds.

U. vulgaris, L. (Common Bladderwort.) Ponds; common.

U. minor, L. Claremont, Dr. Barrows.

U. gibba, L. Claremont, Dr. Barrozes; West Lebanon, Dr. Kénnedy.

U. intermedia, Hayne. Marshes and shallow pools; Canaan, etc.; not rare.

U. purpurea, Walt. Goose Pond, Canaan ; rare.

U. cornuta, Michx. Sandy margins of ponds. Plainfield, Canaan, New London, etc. ; rather cummon. 


\section{ORder 59. VERBENACE开. Vervain Family.}

Verbena, Tourn. Vervain.

V. urticæfolia, L. (White Vervain.) Common.

V. angustifolia, Michx.. In an old pasture near Hanover; rare so far north.

V. hastata, L. (Blue Vervain.) Roadsides; common.

[The Verbenas of the gardens are hybrid forms derived from a number of South American species, including one (V. AUBLETIA) which is indigenous to the West and South.]

Phyrma, L. Lopseed.

P. Leptostachya, L. Woods; common.

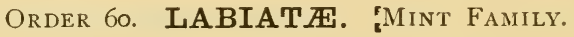

Trichostema, L. Blue-curls. Bastard Pennyroyal.

T. dichotomum, L. Sandy fields.

Teucrium, Tourn. Germander.

T. Canadense, L. (Wood Sage.) Near Conn. river. Hanover, N. H.; Olcott, Vt. ; etc.

Mentha, Tourn. Mint.

M. VIRIDIS, L. (Spearmint.) Wet places ; common ; European.

II. PIPERITA, L. (Peppermint.) Along brooks ; frequent ; European.

M. Canadensis, L. Like the above, in wet places; common.

M. Sativa, L. (Whorled Mint.) Plymouth, Sargent. A garden escape, perhaps.

Lycopus, Tourn. Water Horehound.

L. Virginicus, L. (Bugle-weed.) Marshes.

L. sinuatus, Ell. (L. Europæus, L., var. sinuatus, Gray.) Common in wet grounds.

Pycnanthemum, Michx. Mountain Mint.

P. lanceolatum, Pursh. Claremont, Dr. Barrows; not common.

P. muticum, Pers. Royalton, Vt., Ward.

P. incanum, Michx. Claremont, Dr. Barrowe; rare.

Origanum, Tourn. Wild Marjoram.

O. vulgare, L. Claremont, Dr. Barrozes; rare ; from Europe.

Calamintha, Tourn. Calamint.

C. Clinopodium, Benth. (IVild Basil.) Low woods.

Hedeoma, Pers. American Pennyroyal.

H. pulegioides, Pers. Barren fields. Common.

Monarda, L. Horse-Mint.

M. didyma, L. In old gardens called "Bee Balm;" infrequent in a wild state.

M. punctata, L. (Horse-Mint.) Claremont, Dr. Barrozes ; introduced.

M. fistulosa, L., var. mollis, Benth. (Wild Bergamot.) In gardens; indigenous in Vt., Perkins. 
Blephilia, Raf.

B. hirsuta, Benth. Killington Mt., Vt., Denslow.

Nepeta, L.

N. Cataria, L. (Catnip.) Near dwellings.

N. Glechoma, Benth. (Ground Ivy.) Dooryards, etc. Both species European weeds.

Scutellaria, L. Skullcap.

S. lateriflora, L. (Mad-dog Skullcap.) Wet shady places.

S. galericulata, L. With the preceding; both species common.

Brunella, Tourn. Self-heal.

B. vulgaris, L. Fields; common.

Physostegia, Benth. False Dragon-head.

P. Virginiana, Benth. Hartford, Vt. ; Lebanon, N. H. ; in gardens, sometimes escaping to the roadsides. Native in Vermont.

Marrubium, Tourn. Horehound.

M. VULGaRE, L. Escaped from gardens into waste places; European.

Leonurus, L. Motherwort.

L. CARdiaca, L. Around dwellings; a weed from Europe.

Lamium, L. Dead-Nettle.

L. AMPLEXICAULE, L. In cultivated grounds; rare.

L. maculatum, L. A garden escape; both European.

Galeopsis, L. Hemp-Nettle.

G. Tetrahit, L. (Common Hemp-Nettle.) Waste places; common.

G. Ladanum, I. (Red Hemp-Nettle.) Hanover, etc., Flint ; rare; both European.

Stachys, Tourn. Hedge-Nettle.

S. palustris, L. Near streams.

S. aspera, Michx. (S. palustris, var. aspera, Gray.) Wet grounds; not uncommon.

[Old gardens furnish other Labiate plants, such as OCIMUm BasiLicum (Sweet Basil), Lavandula Vera (Lavender), Hyssopus OFFICINALIS (Hyssop), Thyaus Serpyllum (Creeping Thyme), Satureia hortensis (Summer Savory), Mlelissa officinalis (Balm), SALvia OfFICINALIS (Common Sage), etc. ;-all from Europe.

\section{Order 6i. PLANTAGiNACE无. Plantain Family.}

Plantago, Tourn. Plantain.

P. MAJOR, L. (Common Plantain.) A European weed.

P. Rugellii, Dec. (P. Kamtschatika, Gray's Man., 5th ed.) As common as the preceding and generally mistaken for it, Fesup. (Gray's Flora of $\mathrm{N}$. A., Vol. II, Part I, p. 3 S9.)

P. Lanceolata, L. (Rib-grass. English Plantain.) Donryards and dry fields; common. 


\section{Division III. APETALOUS EXOGENS.}

\section{ORDER 62. ILLECEBRACE无. KNotwort FAMILY.}

(Formerly included in CARYOPHYLLACEÆ.)

Anychia, Michx. Forked Chickweed.

A. capillacea, DC. (A. dichotoma, var. capillacea, Torr.) Dry woods. Geological Survey of $N$. H.

Scleranthus, L. Knawel.

S. AnnuUs, L. Waste places; from Europe.

Order 63. AMARANTACE无. Amaranth FAMily.

Amarantus, Tourn. Amaranth.

A. HYPOCHONDRIACUS, L. (Red Amaranth.) Escaped from gardens.

A. paniculatus, L. A weed in gardens.

A. Retroflexus, L. (Hogweed. Pigweed.) Cultivated ground.

A. Chlorostachys, Willd. (A. retroflexus, var. chlorostachys, Gray.)

Cultivated ground.

var. HYBRIDUs, Watson. (A. retroflexus, var. hybridus, Gray.) All the above from tropical America.

A. albus, L. (Tumble Weed.) A coarse weed.

[Cultivated species of this Order :-CELOSIA CRISTATA (Common Cockscomb), and Gomphrena globosa (Globe Amaranth); both from India.]

Order 64. CHENOPODIACE开. Goosefoot Family.

Chenopodium, Tourn. Pigweed. Goosefoot.

C. polyspermum, L. Claremont, Dr. Barrozes. European.

C. Album, L. (Lamb's Quarters. Pigweed.) Very common. European.

C. URbicum, L. Claremont, Dr. Barrows. European.

C. hybridum, L. (Maple-leaved Goosefoot.) Introduced from New York.

C. Glaucum, L. (Oak-leaved Goosefoot.) European.

C. Bonus-Henricus, L. (Blitum Bonus-Henricus, Reich.)-(Good King Henry.) European.

C. capitatum, Watson. (Blitum capitatum, L.)-(Strawberry Blite.) Around dwellings; infrequent.

C. Botrys, L. (Jerusalem Oak.) Claremont, Dr. Barrows; Hanover, etc.; European.

C. Ambrosioides, L. (Mexican Tea.) Claremont, Dr. Barrows; from tropical America.

Roubieva, Moquin.

R. multifida, Moq. (Chenopodium multifidum, L.) Claremont, Dr. Barrows. All the above plants of this Order are weeds in the vicinity of cultivated grounds or dwellings.

[Cultivated species of this Order include:-BETA vulgaris (Common Beet), and SPINACHIA OLERACEA (Common Spinach), both from Europe.] 
Order 65. PHYTOLACCACE开. Pokeneed FAMily.

Phytolacca, Tourn. Pokeweed.

P. decandra, L. Roadsides. Dr. Barrozes. More common in southern New England.

ORdER 66. POLYGONACE王. BUCKWhEAT FAMILY.

Rumex, L. Dock. Sorrel.

[R. Patientia. L. (Patience Dock.) This plant of northern Vt., and central Mass., should be within our limits.]

R. Britanica, L. (R. orbiculatus, Gray)-(Great Water-Dock.) Swamps. R. altissimus, Wood. (R. Britanica, Gray)-(Pale Dock.) Claremont, Dr. Barrozes.

R. verticillatus, L. (Swamp Dock.) Killington Mt., Vt., Denslow.

R. CRISPUS, L. (Curled Dock.) Very common everywhere.

R. obtusifolius, L. (Bitter Dock.) Fields and roadsides.

R. SAnguineus, L. Bloody-veined Dock.) Royalton, Vt., etc., Ward.

R. Acetosella, L. (Sheep Sorrel.) The last four are weeds in fields and pastures. European.

Rheum, L. Rhubarb.

R. Rhaponticum, L. (Garden Rhubarb or Pie Plant.) Escaped from cultivation. Native of Asia.

Polygonum, Tourn. Knotweed. Smartweed. Bindweed.

P. aviculare, L. (Knotgrass.) Roadsides, dooryards, etc. Common.

P. erectum, L. (P. aviculare, var. erectum, Roth.) With the above.

P. tenue, Michx. Dry fields.

P. Pennsylvanicum, L. Fields; very common.

P. amphibium, L. (Water Smartweed.) Windsor, Vt., Leland; Sharon and Hartford, Vt.; Hanover and Enfield, N. H. ; etc. In or near ponds.

P. Muhlenbergii, Watson. (P. amphibium, var. terrestre, Gray's Man.,

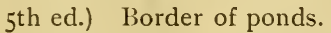

P. ORIEntale, L. (Prince's Feather.) In old gardens; from India.

P. Persicaria, L. (Lady's Thumb.) Fields; European.

P. hydropiperoides, Michx. (Water-pepper.) Shallow water.

P. Hydropiper, L. (Water-pepper.)

P. acre, HBK. (Water Smartweed.)

P. Virginianum, L. Banks of Conn. river, IVindsor, Vt., Leland.

P. arifolium, L. (Halberd-leaved Tear-thumb.) Wet grounds.

P. sagittatum, L. (Arrow-leaved Tear-thumb.) Wet grounds.

P. Convolvulus, L. (Black Bindweed.) Fields; European.

P. cilinode, Michx. On fences, etc. Common.

P. dumetorum, L., var. scandens, Gray. (Climbing False Buckwheat.) Common.

[P. Hartwrightii, Gray. (Common in Vt., Prof. Perkins.) May belong here.]

Fagopyrum, Tourn. Buckwheat.

F. esculentum, Moench. (Buckwheat.) Old fields.

F. TAtaricun, Gaertn. (India Wheat.) Both species cultivated; Asiatic. 
Order 67. ARISTOLOCHIACE开. Birthwort FAMILY.

Asarum, Tourn. Wild Ginger.

A. Canadense, L. Rich woods.

Aristolochia, Tourn. Birthwort.

[A. Sipho, L'Her. (Pipe Vine. Dutchman's Pipe.) A native of Penn. and Va., and sometimes planted for arbors.]

\section{Order 68. LAURACE无. LaUrel Family.}

Sassafras, Nees.

S. officinale, Nees. Plainfield, N. H., Miss Stevens; Springfield, Vt., Rev.

S. Hayward; Charlestown, N. H.; Randolph, Orange, and Pomfret, Vt.; rare; southward it is common.

Lindera, Thunb. Spice-wood. Benjamin-bush.

[L. Benzoin, Blume. A shrub found in Vermont (Prof. Perkins) and probably within our limits.]

\section{ORder 69. THYMEL无ACE开. MEZEREUM FAMILy.}

Dirca, L. Leatherwood.

D. palustris, L. Rich woods; sometimes in swamps, common.

Daphne, L. Mezereum.

[D. Mezereum, L. An early blooming garden shrub from Europe.]

ORder 70. LORANTHACE开. Mistletoe Family.

Arceuthobium, Bieb.

A. pusillum, Peck. (Dwarf Mistletoe.) Found parasitic on Picea nigra (Black Spruce) in a swamp in Lebanon ( $188_{3}$ ); also near Bear Pond, Canaan ( $\mathrm{ISS}_{3}$ ), Fesup. Probably rare. It is also found in the Adirondack region of $\mathrm{N}$. Y., and is very injurious.

Order 7 I. SANTALACE无. Sandalwood Family.

Comandra, Nutt. Bastard Toad-flax.

C. umbellata, Nutt. Canaan, F. B. Wallace.

ORdER 72. EUPHORBIACE无. SPURGE FAMILY.

Euphorbia, L. Spurge.

E. maculata, L. A common weed in gardens.

E. Preslii, Guss. (E. hypericifolia, Gray's Man., 5th ed.) Roadsides and gardens.

E. Cyparissias, L. (Cypress Spurge.) Roadsides. Often seen in cemeteries.

E. Peplus, L. Dooryards. Hanover, fesup. The last two are European. [E. Marginata, Pursh., from the West, is cultivated.]

Acalypha, L. Three-seeded Mercury.

A. Virginica, L. A weed in cultivated ground; frequent. 
Ricinus, L. Castor-oil Plant.

[R. COMMUNIS, L. Very common in cultivation.]

Order 73. URticaCe无. Nettle Family.

Ulmus, L. Elm.

U. fulva, Michx. (Red Elm. Slippery Elm.) Banks of streams; not rare.

U. Americana, L. (Common Elm. American or White Elm.) Abundant.

U. racemosa, Thomas. (Corky White Elm.) Meriden, Dr. Barrows, where a few large trees are found, apparently self sown. It is sometimes planted, and at the West is a valuable tree and indigenous.

Celtis, Tourn. Hackberry. Nettle Tree. Sugarberry.

C. occidentalis, L. Banks of Conn. river throughout the district, but not abundant.

Cannabis, Tourn.

C. SAtiva, L. (Hemp.) Roadsides; escaped from cultivation; European.

Humulus, L. Hopvine.

H. Lupulus, L. Roadsides; frequent in cultivation.

Urtica, Tourn. Nettle.

U. gracilis, Ait. (Common Nettle.) Fence rows; common.

U. URens, L. Claremont, N. H., Dr. Barrows: Royalton, Vt., Ward; scarce.

Laportea, Gaudichaud.

L. Canadensis, Gaudich. (Wood-Nettle.) Rich woods.

Pilea, Lindl.

P. pumila, Gray. (Clearweed.) In shady places near divellings.

Bœhmeria, Jacq. False Nettle.

B. cylindrica, Willd. Shady and moist ground.

[Morus RUbra, L., the Red Mulberry tree, is a native of central New England. MORUS NIGRA from the Levant with black fruit, and the whitefruited MORUS ALBA from China are occasionally planted.]

\section{Order 74. PLAtanaCE无. Plane-tree Family.}

Platanus, L. Plane-Tree.

P. occidentalis, L. (Buttonwood or American Sycamore.) Meriden and Hanover, N. H.; Hartford, Hartland, and Sharon, Vt. Not abundant so far north, and commonly planted.

ORDER 75. JUGLANDACE无. IVALnU't Family.

Juglans, L. Walnut.

J. cinerea, L. (Butternut.) Very common.

[J. NIGRA, L., the Black Walnut of the IVest, is often seen in private grounds.] 
Carya, Nutt. Hickory.

C. alba, Nutt. (Shell-bark Hickory.) Claremont, N. H., Dr. Barrowe; Royalton, Vt., L. Wild.

C. porcina, Nutt. (Pig-nut. Hog Walnut.) Common.

C. amara, Nutt. (Bitter-nut.) Claremont and Lebanon, Dr. Barrows. "Along the Conn. river on both sides," Flint.

\section{Order 76. MYRICACE无. Siveet-Gale Family.}

\section{Myrica, L.}

M. Gale, L. (Sweet Gale.) Swamps; Hanover, Sunapee Lake, etc.; not uncommon.

M. asplenifolia, Endl. (Comptonia asplenifolia, Ait.)-(Sweet Fern.) Dry hills ; common.

\section{Order 77. CUPULIFER Æ. OAK Family.}

(Including Betulaceæ, The Birch Fanily.)

Betula, Tourn. Birch.

B. lenta, L. (Black or Cherry Birch.) Throughout our limits, but less. common north of Hanover.

B. lutea, Michx. f. (Yellow Birch.) Common.

B. populifolia, Ait. (B. alba, var. populifolia, Spach.)-(American White Birch. Gray Birch.) Not abundant, but very common in the centre of the state.

B. ALBA, L. (European White Birch.) Introduced at Hanover, where it spreads rapidly.

B. papyrifera, Marsh. (B. papyracea, Ait.)-(Paper or Canoe Birch. White Birch of northern New England.) Very common.

Alnus, Tourn. Alder.

A. viridis, DC. (Green or Mountain Alder.) Banks of Conn. river, Hanover, etc.

A. incana, Willd. (Speckled Alder.) Common northward.

A. serrulata, Ait. (Smooth Alder.) Claremont, Dr. Graves. More abundant southward.

Corylus, Tourn. Hazelnut.

C. rostrata, Ait. (Beaked Hazelnut.) Common.

C. Americana, Walt. (Wild Hazelnut.) Hartford, Vt., and Hanover, N. $\mathrm{H}$.; not uncommon.

Ostrya, Micheli. Hop-Hornbeam.

O. Virginica, Willd. (Lever-wood. Iron-wood.) Common.

Carpinus, L. Hornbeam. Iron-wood.

C. Caroliniana, Walt. (C. Americana, Michx.)-(American Hornbeam. Blue or Water Beech.) Common.

Quercus, L. Oak.

Q. alba, L. (White Oak.) More or less common on both sides of the Conn. river. 
Q. coccinea, Wang., var. tinctoria, Gray. (Black Oak.) North Charlestown, Flint; and in a few towns in both states.

Q. rubra, L. (Red Oak.) The most abundant species. Other species on our borders may yet be found within our limits.

Castanea, Tourn. Chestnut.

C. sativa, Mill., var. Americana. (C. vesca, var. Gray's Man., 5th ed.) Infrequent. Claremont is nearly the northern limit; frequently planted and growing well in sheltered places.

Fagus, Tourn. Beech.

F. ferruginea, Ait. Varies greatly; wood light colored or reddish, as grow ing on dry or moist ground. Abundant.

\section{ORder 78. SALICACE无. Willow Family.}

Salix, Tourn. Willow.

S. nigra, Marsh. (Black Willow.) The only native species tree-like insize.

S. lucida, Muhl. (Shining Willow.) IVet places; very handsome shrub.

S. FRAGIlis, L. (Brittle Willow.) Large tree; from Europe ; early introduced.

S. ALBA, L. (White Willow.) Both this and the above with many varieties, and hybrid forms.

S. BABylonicA, Tourn. (Weeping Willow.) From Asia, and here known only in the pistillate form.

S. longifolia, Muhl. (Long-leaved Willow.) Banks of the Conn. river at Lebanon, N. H., and IVindsor, Vt. Not very common.

S. rostrata, Richard. (S. livida, Wahl., var. occidentalis, Gray.)-(Beaked Willow.) Common.

S. discolor, Muhl. (Glaucous Willow.) Very common everywhere.

S. humilis, Marsh. (Prairie Willow.) Dry ground; common.

S. tristis, Ait. (Dwarf Gray Willow.) With the above.

S. sericea, Marsh. (Silky Willow.) Not rare.

S. petiolaris, Smith. With the preceding.

S. Purpurea, L. (Purple Willow.) From Europe; used for basket-work.

S. cordata, Muhl. (Heart-leaved Willow.) Very common and variable.

[In addition to the above, other species may occur on our mountains and elsewhere. Numerous hybrid forms have been noted, making the precise determination of the species at times impracticable. SALIX FRAGILIS and S. ALBA are much intercrossed. S. DISCOLOR and S. HUMILIS hybridize, and small forms of the latter are not readily distinguished from S. TRISTIS. See description of Genus in Manual, 6th ed. by M. S. BEBB.]

Populus, Tourn. Poplar. Aspen.

[P. ALBA, L. (IVhite Poplar. Abele Tree.) Cultivated from Europe, and in the pistillate form only.]

P. tremuloides, Michx. (American Aspen.) Everywhere common.

P. grandidentata, Michx. (Great-toothed Poplar.) Frequent. 
P. balsamifera, L. (Balsam Poplar.) Abundant near streams. var. CANDICANS, Gray. (Balnı of Gilead.) Commonly cultivated never spontaneous within this district, and perhaps European.

[P. MONIliferA, Ait. (Cotton-wood.) In cultivation from the IVest; rarely spontaneous ]

[P. Dilatata, Ait. (Lombardy Poplar.) From Europe, in the pistillate form only, and freely spreading by suckers.]

\section{ORder 79. EMPETRACE无. CRowberry FAMILY.}

Empetrum, Tourn. Crowberry.

E. nigrum, L. (Black Crowberry.) Moosilauke Mt., Rev. F. Blake.

ORdER 8O. CERATOPHYLLACE无. Hornwort FAMLY.

Ceratophyllum, L. Hornwort.

C. demersum, L. Goose Pond in Canaan, George Pond in Enfield, N. H. ; Fairlee Pond, Vt. ; etc. Not rare. 


\section{SUbCLASS II. GYMNOSPERMÆ.}

\section{Order 8r. CONIFER 无. Pine Family.}

Pinus, Tourn. Pine.

P. Strobus, L. (White Pine.) Very common; the most valuable species.

P. rigida, Miller. (Pitch Pine.) Sandy soil; more common farther south.

P. resinosa, Ait. (Red or Norway Pine.) Sparingly found throughout our limits. "It is not a native of Europe, but was named from the town of Norway in Maine." Robert Douglass.

P. sylvestris, L. (Scotch Pine. Scotch Fir.) Already spontaneous in Hanover.

Picea, Link. Spruce.

P. nigra, Link. (Abies nigra, Poir.)-(Black Spruce.) ' Swamps and cold mountain woods; common.

var. rubra, Engelm. With the above, "cones larger, bright red-brown."

Better timber than the type, and called by some lumbermen "Yellow Spruce."

P. alba, Link. (Abies alba, Michx.)-(White Spruce.) Newport, N. H., Dr. Graves; Windsor, Vt., Leland; Killington Mt., Vt., Denslow ; and more or less throughout our limits.

Tsuga, Carrière. Hemlock.

T. Canadensis, Carr. (Abies Canadensis, Michx.)-(Hemlock.) Common. Abies, Link. Fir.

A. balsamea, Miller. (Balsam Fir.) Damp woods and mountain swamps; common.

Larix, Tourn. Larch.

L. Americana, Michx. (American Larch. Tamarack. Hackmatack.) Cold swamps; common.

L. EuRopæA, L. (European Larch.) Widely planted, and in Hanover spreading freely.

Thuya, Tourn. Arbor Vitæ.

T. occidentalis, L. Commonly called White Cedar. Common in swamps and on river-banks.

Juniperus, L. Juniper.

J. communis, L. (Common Juniper.) Claremont (Dr. Barrows), Hanover, Orford, Enfield, Sutton, etc., N. H.; Pomfret, Orange, Williamstown, Vt.; etc.

J. Virginiana, L. (Red Cedar.) Hanover, N. H.; Fairlee, Vt., $F . O$. Grover; and in many places in the district, but not common.

Taxus, Tourn. Yew.

T. Canadensis, Willd. (T. baccata, var. Canadensis, Willd.)-(Ground Hemlock.) Common.

[Among evergreens in cultivation will be found:-PInUs AUSTRIACA (Austrian Pine), Picea excelsa (Norway Spruce), Abies pectinata (European Silver Fir), etc.] 


\section{Class II. MONOCOTYLEDONOUS OR ENDOGENOUS PLANTS.}

\section{ORDER S2. HYDROCHARIDACE无. FROG'S-BIT FAMILY.}

Elodea, Michx. Water-weed.

E. Canadensis, Michx. (Anacharis Canadensis, Planchon.) Found in slow streams and ponds. In a pool near Conn. river, Norwich, Vt. ; Dr. Blanchard; and doubtless elsewhere within our limits.

Vallisneria, L. Tape-Grass. Eel-Grass.

V. spiralis, L. Hanover, N. H., in Conn. river; Fairıee Pond, Vt.; etc.

\section{ORDER S3. ORCHIDACEÆ. ORCHIS FAMILY.}

Microstylis, Nutt. Adder's-Mouth.

M. monophyllos, Lindl. Hanover, N. H.; Pomfret, Union Village, and Ascutney Mt., Vt.

M. ophioglossoides, Nutt. Shrewsbury and Killington Mts., Vt., Denslow ; Pomfret and Ascutney Mt., Vt.; New London, N. H., Sargent. Both species in wet woods.

Liparis, Richard. Twayblade.

L. liliifolia, Richard. Banks of the Conn, river at Windsor, Vt., Leland.

L. Lœselii, Richard. Bogs and hillside pastures, Hanover, Meriden, Plymouth, etc.; not rare.

Calypso, Salisb. Calypso.

[C. borealis, Salisb. This rare and local plant of northern Vermont, one of the most beautiful of its Order, is said by Dr. Blanchard to be found in cold cedar swamps throughout Caledonia and Essex counties. He has himself collected it in Ryegate, Barnet, and Peacham, just beyond our northern limits, and it can hardly fail of being within these limits.]

Aplectrum, Nutt. Putty-root. Adam-and-Eve.

A. hiemale, Nutt. Walpole and Claremont, Dr. Barrows; New London, $I V . F$. Carter. In rich, dark woods. Very rare.

Corallorhiza, Haller. Coral-root.

C. innata, R. Jir. Damp woods ; not rare.

C. odontorhiza, Nutt. Norwich, Vt.; rare.

C. multiflora, Nutt. Rich woods. Hanover, etc., and on both sides of the Conn. river.

Listera, R. Brown. Twayblade.

L. cordata, R. Br. Moosilauke Mt., N. H., Flint; Killington Mt., Vt., Denslow.

L. convallarioides, Nutt. Mountain bogs.

Spiranthes, Richard. Ladies' Tresses.

S. latifolia, Torr. Moist banks. Windsor, Vt., Leland; Union Village, Vt., Blanchard; Norwich, Vt., Sargent; Royalton, Vt., Ward. 
S. Romanzoffiana, Cham. North Pomfret, Vt., Morgan; Royalton, Vt., Ward; Grantham, N. H., Barton; New London, N. H., Carter.

S. cernua, Richard. Wet meadows; common.

S. gracilis, Big. Sandy soil. Hanover and Plymouth, N. H.; Norwich and Royalton, Vt. ; etc. ; not rare.

Goodyera, R. Br. Rattlesnake-Plantain.

$\mathrm{G}$. repens, R. Br. In rich woods; not rare.

G. pubescens, R. Br. Rich woods; frequent.

Arethusa, Gronov. Arethusa.

A. bulbosa, L. Near Goose Pond in Canaan, N. H., Dr. Barrozus; Peat bogs, Hanover, N. H., Fesup; Pine Hill Ponds, Rutland, Vt., Denslowv; rare.

Calopogon, R. Br. Calopogon. Grass Pink.

C. pulchellus, R. Br. "Bogs and moist pastures.

Pogonia, Juss. Pogonia.

P. ophioglossoides, Nutt. Bogs. Bear and Mud Ponds, Canaan; George Pond,-Enfield; Hanover, etc., N. H. ; IVaterman's Pond near Union Village, Vt. ; etc.

\section{Orchis, L.}

O. spectabilis, L. (Showy Orchis.) Rich woods; not rare.

[O. rotundifolia, Pursh. (Habenaria rotundifolia, Richard.) Sphagnous bogs, Perkins's Vermont Flora. This rare species is likely to occur on our northern borders.]

Habenaria, Willd. Rein-Orchis.

H. tridentata, Hook. Rich and moist woods; not rare.

H. virescens, Spreng. (Greenish Orchis.) Claremont, Dr. Barrows.

H. bracteata, R. Br. (H. viridis, var. bracteata, Reichenb.)-(Bracted Green Orchis.) Rich woods.

H. hyperborea, R. Br. (Northern Green Orchis.) Cold bogs, also wet woods.

H. dilatata, Gray. (Northern White Orchis.) Pomfret, Sharon, Norwich, Windsor, and Killington Mt., Vt.; Hanover, N. H.; etc. IVet grounds.

H. obtusata, Rich. Killington Mt., Vt., Morgan, Densloze.

H. Hookeri, Torr. (Smaller Round-leaved Orchis.) Norwich and Sharon, Vt., Morgan; Hanover and Lyme, N. H. Woods; not rare.

H. orbiculata, Torr. (Larger Round-leaved Orchis.) Mountain woods; not uncommon.

H. blephariglottis, Torr. (WWhite Fringed Orchis.) Grantham Mt., and New London, N. H.; Royalton, Vt.; etc.

H. lacera, R. Br. Moist ground; common.

H. psycodes, Gray. (Purple Fringed Orchis.) Sivamps; more common than the next.

H. fimbriata, R. Br. (Purple Fringed Orchis.) Claremont, Dr. Barrows ; New London, Sargent. Spike not so dense, and flowers larger than the preceding, and blooming two weeks earlier. 
Cypripedium, L. Lady's Slipper. Moccasin-Flower.

C. arietinum, R. Br. (Ram's-Head. Lady's Slipper.) Ascutney Mt., Vt., Dr. Phelps; Lyme, N. H., R. S. Alden; Hanover, N. H., near Olcott Falls, E. P. Barrows: very rare.

C. parviflorum, Salisb. (Smaller Yellow Lady's Slipper.) Sometimes with the next, but not readily distinguished from it.

C. pubescens, Willd. (Larger Yellow Lady's Slipper.) Hanover, N. H.; Norwich, Vt.; frequent.

C. spectabile, Swartz. (Showy Lady's Slipper.) Swamps. Hanover, N. H., F. H. Herrick; Lebanon, N. H., Dr. Barrowes; Sharon, Vt., Dr. Baxter; Barnard, Vt., Ward. Not very abundant.

C. acaule, Ait. (Stemless Lady's Slipper.) Dry or moist woods; common.

\section{ORDER 84. IRIDACE无. IRIS FAMILY.}

Iris, Tourn. Blue-Flag.

I. versicolor. L. Swamps; common.

Sisyrinchium, L. Blue-eyed Grass.

S. angustifolium, Mill. (S. Bermudiana, var, mucronatum, Gray.) Moist meadows; common.

S. anceps, Cav. (S. Bermudiana, var. anceps, Gray.) Same range as the above.

[Garden plants of this Order belong to the following Genera :-IRIS, TigRidia (Tiger-flower), Gladiolus (Sword Lily), and CROCUS.]

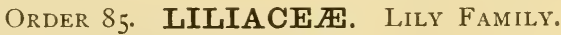

Smilax, Tourn. Greenbrier. Cat-brier.

S. herbacea, L. (Carrion Flower.) River-banks; not infrequent.

S. rotundifolia, L. (Greenbrier.) Thickets; not common.

Allium, L. Onion.

A. tricoccum, Ait. (Wild Leek.) Rich woods; frequent on both sides of the Conn. river.

A. Schœnoprasum, L. (Chives.) Banks of the Conn. river at Windsor, Vt., Leland. In old gardens.

A. Canadense, Kalm. (WVild Garlic.) Moist meadows.

Ornithogalum, Tourn. Star-of-Bethlehem.

O. umbellatum, L. Dooryards; coinmon; European.

Hemerocallis, L. Day-lily.

H. FULVA, L. Koadsides, frequent and well established; from Europe.

Convallaria, L. Lily of the Valley.

[C. Majalis, L. Common in gardens; a native of Virginia, and sometimes escaping to the roadsides. Our plant is of European origin.]

Polygonatum, Tourn. Solomon's Seal.

P. biflorum, Ell. (Common Solomon's Seal.) Wooded banks and hillsides; common. 
P. giganteum, Dietrich. (Great Solomon's Seal.) River-banks; rare; Hartford, Vt., IVard.

Asparagus, Tourn. Asparagus.

A. Officinalis, L. A common garden escape; European.

Smilacina, Desf. False Solomon's Seal.

S. racemosa, Desf. (False Spikenard.) Moist copses.

S. stellata, Desf. Both banks of Conn. river; Plymouth, Miss Langdon; etc.

S. trifolia, Desf. Sphagnous swamps; somewhat rare.

Maianthemum, Wigg.

M. Canadense, Desf. (Smilacina bifolia, var. Canadensis, Gray.) Moist woods; very common.

Streptopus, Michx. Twist-Foot. Twisted-Stalk.

S. amplexifolius, DC. Claremont, Groton, Cube Mt., and Moosilauke Mt., N. H.; Pomfret and Killington Mt., Vt. ; etc.

S. roseus, Michx. Frequent in damp woods.

Clintonia, Raf. Clintonia.

C. borealis, Raf. Moist woods; common.

Uvularia, L. Bellwort.

U. perfoliata, L. Claremont, Dr. Barrows; scarce.

$\mathrm{U}$. grandiflora, Smith. Rich woods; not uncommon.

Oakesia, Watson. Sessile-leaved Bellwort.

O. sessilifolia, WVatson. (Uvularia sessilifolia, L.) Common in low woods.

Erythronium, L. Adder's-Tongue.

E. Americanum, Ker. (Yellow Adder's-Tongue.) Very common in rich woodlands.

Lilium, L. Lily.

L. Philadelphicum, L. (Orange Lily.) Common in sandy, open woods.

L. Canadense, L. (Yellow Lily.) Moist meadows; very abundant.

[L. TIGRINUM, Ker. (Tiger Lily.) Sometimes spontaneous on the roadside. From Eastern Asia.]

Medeola, Gronov. Indian Cucumber-root.

M. Virginiana, L. Rich moist woods; common.

Trillium, L. Wake Robin. Birthroot.

T. erectum, I. (Early Trillium.) Common in rich woods.

var. album, Pursh. Hartford, Vt., C. H. Hazen; Chittenden, Vt., abundant, Flint. Found with the type.

T. grandiflorum, Salisb. (Large-flowered Trillium.) The finest species of the genus, and common in Vermont west of the Green Mits. It has been reported from Woodstock and Thetford, Vt., and should be looked for elsewhere on our western border.

T. cernuum, L. (Nodding Trillium.) Claremont, Hanover, and Lebanon, N. H.; Norwich, Vt., H. A. Nichols. Not abundant.

T. erythrocarpum, Michx. (Painted Trillium.) Moist woods; common. 
Tofieldia, Hudson. False Asphodel.

T. glutinosa, Willd. Quechee Gulf, Vt., Leland; very rare.

Veratrum, Tourn. False Hellebore.

V. viride, Ait. (American White Hellebore. Indian Poke.) Low grounds ; common.

[The following Liliaceous plants are found in gardens:-CoLCHICUM AUTUMNALE from Europe, LILIUM of many species from Europe and the East, FritTillaria imperialis (Crown Imperial) from Asia, Tulipa GesNERIANa (Common Tulip of many varieties) also Asiatic, Scilla (Squill) of several European species, MUSCARI BOTRYoIDes (Grape Hyacinth) from Europe, Hyacinthus orientalis (Common Hyacinth) of the Levant, FUnkia (Day Lily) from Europe and the East, YUCCA FILAMENTOSA of the south-western United States, etc.]

\section{Order 86. PONTEDERIACE无. Pickerel-weed Family.}

Pontederia, L. Pickerel-weed.

P. cordata, L. Ponds; common and trimorphous.

ORDER S7. XYRIDACE开. Yellow-EYED-GRASS FAMILY.

Xyris, Gronov. Yellow-eyed Grass.

X. flexuosa, Muhl. The typical form not yet reported.

var. pusilla, Gray. Sandy margins of streams and ponds. Meriden, Canaan, etc., Fesup.

ORder 88. JUNCACE无. RUSH FAMILY.

Juncus, Tourn. Rush.

J. effusus, L. Marshes. A large and very common species.

J. filiformis, L. Low grounds, not very rare.

J. trifidus, L. Moosilauke Mt., Rev. F. Blake.

J. marginatus, Rostk. Sands of Conn. river.

J. Greenii, O. \& T. Haverhill, Flint.

J. tenuis, Willd. Very common.

J. bufonius, L. (Toad Rush.) Roadsides.

J. pelocarpus, E. Meyer. Shores of Enfield Pond, Fesup.

J. articulatus, L. Wet grounds.

J. acuminatus, Michx. Wet places; common and very variable.

J. nodosus, L. Swamps and banks of streams.

J. Canadensis, J. Gay. Common; a late blooming species.

var. longicaudatus, Engelm.

var. coarctatus, Engelm.

Luzula, DC. Wood-Rush.

L. vernalis, DC. (L. pilosa, Willd.) Hanover, A. C. French; Canaan, Dr. Barrows; not uncommon.

L. spadicea, DC., var. melanocarpa, Meyer. (L. parviflora, var. melanocarpa, Gray.) Moosilauke Mt., Rev. F. Blake.

L. campestris, DC. Meadows ; common.

L. spicata, Desv. Moosilauke Mt., Rev. F. Blake. 
Order S9. TYPHACE无. CAT-tall Family.

Typha, Tourn.

T. latifolia, L. (Common Cat-tail.)

Sparganium, Tourn. Bur-reed.

S. simplex, Huds. Borders of ponds and streams.

var. androcladum, Engelm. The largest form; common.

var. fluitans, Engelm. Not uncommon in ponds and streams.

var. angustifolium, Engelm. In ponds.

S. minimum, Fries. Pond holes, Norwich, Vt., Zesup; probably not rare.

Order 90. ARACE无. Arum Family.

Arisæma, Martius.

A. triphyllum, Torr. (Indian Turnip.) Common in rich woods.

A. Dracontium, Schott. (Dragon Arum.) Charlestown, Dr. Graves; rare.

Peltandra, Raf. Arrow Arum.

P. undulata, Raf. (P. Virginica, Kunth.) Mud Pond, Canaan, Fesup; Enfield, Atwell. Perhaps not very common.

Calla, L. Water Arum.

C. palustris, L. Bugs. Claremont and Newport, Dr. Barrows; New London, N. H., Carter; Hartford, Springfield, and Sherburne, Vt. ; not very common.

Symplocarpus, Salisb. Skunk Cabbage.

S. fœtidus, Salisb. Wet places. Norwich and Thetford, Vt.; etc. Not as common as it is farther south.

Acorus, L. Sweet Flag. Calamus.

A. Calamus, L. Swamps; everywhere.

ORDER 9I. LEMNACE无. DUCKWEed FAMILY.

Lemna, L. Duckweed.

L. minor, L. Floating in still water; not infrequent in ponds and ditches. Hanover and Enfield, N. H. ; Norwich, Vt. ; etc.

Spirodela, Schleid. Duckweed.

S. polyrrhiza, Schleid. (Lemna polyrrhiza, L.) In the same localities as the preceding, and like it rarely ever found in flower or fruit; propagating by winter buds. This species is less common than the preceding.

\section{Order 92. ALISMACE无. Water-Plantain Family.}

Alisma, L. Water-Plantain.

A. Plantago, L. Shallow water; abundant and variable.

Sagittaria, L. Arrow-head.

S. variabilis, Engelm. In wet places; several varieties.

S. graminea, Michx. Lebanon, Hanover, etc. ; not rare near ponds. 
Order 93. NAIADACE开. Pondweed Family.

Scheuchzeria, L.

S. palustris, L. Cranberry Pond on Grantham Mt., N. H.; Waterman's: Pond near Union Village, Vt.; etc.; Fesup.

Potamogeton, Tourn. Pondweed.

P. natans, L. Ponds and streams.

P. Oakesianus, Robbins. Cranberry Pond on Grantham Mt.

P. Pennsylvanicus, Cham. (P. Claytonii, Tuckerm.) Common in ponds and streams.

P. Spirillus, Tuckerm. Streams and ponds.

P. hybridus, Michx. Shallow ponds.

P. rufescens, Schrad. Windsor, Vt. ; infrequent.

P. fluitans, Roth. (P. lonchites, Tuck.) Conn. river; common.

P. amplifolius, Tuckerm. Ponds and large rivers.

P. heterophyllus, Schreb. (P. gramineus, Fries.) Conn. river; common and variable.

P. perfoliatus, L. Conn. river; common. var. lanceolatus, Robbins. Conn. river.

P. zosteræfolius, Schum. (P. compressus, Fries.) Randolph, Vt., $7 . W$. Freley; Fairlee Pond, Vt.; George Pond, Enfield, N. H.

P. pauciflorus, Pursh. Pools.

P. pusillus, L. Shallow pools; not rare.

P. Tuckermani, Robbins. Claremont, Dr. Barrozes; scarce.

P. pectinatus, L. In Conn. river; abundant.

P. Robbinsii, Oakes. Mascoma Creek near IVest Lebanon, and George Pond, Enfield, N. H.; Fairlee Pond, Vt. Common.

[Other species than the above are probably within our limits.]

Zannichellia, Micheli. Horned Pondweed.

Z. palustris, L. Ponds. Claremont, Dr. Barrows; scarce.

Naias, L. Naiad.

N. flexilis, Rostk. \& Schmidt. Ponds; rather common.

Order 94. ERIOCAULE无. Pipeivort FAMILy.

Ericaulon, L. Pipewort.

E. septangulare, With. Sandy borders of ponds; common.

Order 95. CYPERACE无. Sedge Family.

Cyperus, Tourn. Galingale.

C. diandrus, Torr. Low ground, and variable.

C. aristatus, Rottb. (C. inflexus, Muhl.) Shores of Conn. river.

C. filiculmis, Vahl. Dry, sterile soil ; common.

C. dentatus, Torr. Margins of ponds.

C. esculentus, L. (C. phymatodes, Muhl.) Low ground, spreading by root-like tubers.

C. strigosus, L. Very common. 
Dulichium, Pers.

D. spathaceum, Pers. Marshes; common.

Eleocharis, R. Br. Spike-Rush.

E. Robbinsii, Oakes. In ponds, rather rare; George Pond, Enfield; etc.

E. ovata, R. Br. (E. obtusa, Schultes.) Wet places.

E. olivacea, Torr. Mouth of Mascoma river, Lebanon, Fesup.

E. palustris, R. Br. Wet places, and very variable in size, etc.

E. intermedia, Schultes. N. Pomfret, Vt., A. P. Morgan.

E. tenuis, Schultes. Wet places; very common.

E. acicularis, R. Br. Muddy shores; abundant.

Fimbristylis, Vahl.

F. autumnalis, Roem. \& Schultes. Low grounds.

F. capillaris, Gray. Sandy fields.

Scirpus, Tourn. Bulrush or Club-Rush.

S. pungens, Vahl. Borders of ponds; common.

S. Torreyi, Olney. Borders of ponds.

S. lacustris, L. (S. validus, Vahl.)-(Great Bulrush.) Very common.

S. debilis, Pursh. Near ponds throughout the district.

S. sylvaticus, L., var. digynus, Boeckl. (S. microcarpus, Presl.) Along brooks; common.

S. atrovirens, Muhl. Wet meadows; common.

Eriophorum, L. Cotton-Grass.

E. cyperinum, L. (Scirpus Eriophorum, Michx.) Wet meadows; quite variable ; lateral spikelets sometimes long pedicelled.

E. alpinum, L. Cold bogs. Not rare; an early species.

E. vaginatum, $L$. Peat bogs, Lebanon; not very common.

E. Virginicum, L. Low meadows; a variety has white wool.

E. polystachyon, L. Low meadows. Somewhat variable; very common.

E. gracile, Koch. Low meadows; frequent.

Rhynchospora, Vahl, Beak-Rush.

R. fusca, Roem. \& Schultes. Cranberry Pond on Grantham Mt.; rare.

R. alba, Vahl. Bogs ; common.

R. glomerata, Vahl. Low grounds; common.

Cladium, P. Browne. Twig-Rush.

C. mariscoides, Torr. Common in bogs.

Carex, Ruppius. Sedge.

C. aquatilis, Wahl. $(35)^{1}$ Low grounds, Dr. Barrows.

C. arctata, Boott. (5I) Woods; common.

C. aurea, Nutt. (79) Moist banks; not rare.

C. bromoides, Schk. (I 24) Bogs; common.

C. bullata, Schk. (I4) Canaan, Dr. Barrozes; rare.

C. canescens, L. (II9) Swamps; common.

1 The number following each name refers to its position in Gray's Manual of Botany, 6th edition. 
var. vulgaris, Bailey.

var. alpicola, Wahl. (C. vitilis, Fries.)

C. castanea, Wahl. (49)-(C. flexilis, Rudge.) Pomfret, Vt., Morgan.

C. cephalophora, Muhl. (I I4) Fields; very abundant.

C. communis, Bailey. (89)-(C. varia of Man., 5th ed.) Hillsides; common.

C. conoidea, Schk. (65) Moist meadows.

C. crinita, Lam. (4I)-(C. gynandra, Schw.) Swamps; variable.

C. debilis, Michx., var. Rudgei, Bailey. (52)-(C. debilis, Man., 5th ed.) Somewhat variable; common.

C. Deweyana, Schw. (123) Woods and clearings; frequent.

C. digitalis, Willd. (69) Hillsides.

C. echinata, Murray, var. microstachys, Boeckl. (I IS)-(C. scirpoides, Schk. and C. sterilis, Willd.) Very common and variable.

C. filiformis, L. (27) Peat bogs; frequent.

var. latifolia, Boeckl. (C. lanuginosa. Michx.) Low meadows; common.

C. flava, L. (62) Wet meadows; common.

var. viridula, Bailey. (C. (Ederi, Ehrh.) Vermont:-banks of White

River, Flint; Fairlee Lake, Blanchard; Pomfret, Morgan.

C. fœnea, Willd. (I 30$)-($ C. adusta, Boott.) In clearings. Claremont, etc., Fesup.

C. folliculata, L. (4) Bogs; frequent.

C. formosa, Dewey. (56) Claremont, Dr. Barrows.

C. gracillima, Schw. (55) Wet meadows; abundant.

C. granularis, Muhl. (60) Lebanon, N. H., Dr. Barrows; in Vermont also, Dr. Blanchard.

C. grisea, Wahl. (58) Moist grounds.

C. Hitchcockiana, Dewey. (67) Pomfret, Vt., A. R. Morse; rare.

C. Houghtonii, Torr. (26) Norwich, Vt., E. A. Edmands; rare.

C. hystricina, Muhl. (I8) Wet meadows; frequent.

C. intumescens, Rudge. (5) Swamps; very common.

C. laxiculmis, Schw. (70)-(C. retrocurva, Dewey.) Woods.

C. laxiflora, Lam. (68) Woods; several varieties; common.

C. lenticularis, Michx. (36) Shores of Enfield Pond, Dr. Kennedy ; Baker's Pond, Orford, Grover.

C. limosa, L. (45) Peat bogs; not uncommon.

C. longirostris, Torr. (48) Lebanon, H. C. Fall. Frequent.

C. lupulina, Muhl. (7) Wet grounds; rather common, and variable.

C. lurida, Wahl. (I6)-(C. tentaculata, Muhl.) Meadows; very common, and variable; hybridizes with the preceding.

C. Magellanica, Lam. (43)-(C. irrigua, Smith.) Norwich, Vt.; bogs.

C. monile, Tuckerm. (I2) Moist grounds; frequent.

C. Muhlenbergii, Schk. (I I2) Sterile soil.

C. pallescens, L. (63) Meadows; common.

C. pauciflora, Lightf. (I) Sphagnous bogs. Plainfield, Dr. Barrows. Rare. 
C. pedunculata, Muhl. (82) Dry woods; common.

C. Pennsylvanica, Lam. (88) Woods and hillsides; very common, and blooming early.

C. plantaginea, Lam. (74) Rich hillside woods; common.

C. platyphylla, Carey. (72) Rich hillside woods; common.

C. polytrichoides, Muhl. (96) Swamps; abundant.

C. prasina, Wahl. (39)-(C. miliacea, Muhl.) Bogs; not common. Borders of streams, Dr. Barrows.

C. Pseudo-Cyperus, L. (19) Goose Pond in Canaan, Dr. Barrows. var. Americana, Hochst. (C. comosa, Boott.) Wet places.

C. pubescens, Muhl. (92) Lebanon, Dr. Barrows.

C. retrorsa, Schw. (1 5) Marshes, near streams. var. Hartii, Gray. Frequent.

C. riparia, Curtis. (29) Borders of ponds, etc.; common.

C. rosea, Schk. (I Io) Woods and meadows; very variable.

C. scabrata, Schw. (23) Swamps; common.

C. scoparia, Schk. (I2S) Meadows; common and variable.

C. sparganioides, Muhl. (III) Low grounds; frequent.

C. stipata, Muhl. (IOI) Low groureds; common.

C. straminea, Willd. (1 32$)$-(C. straminea, var. tenera, $5^{\text {th }}$ ed. of Man.) Copses and fields; extremely variable. (See Manual.)

C. stricta, Lam. (34) Wet meadows; very abundant. var. decora, Bailey. (C. aperta, Boott.) Not as common as the type.

C. tenella, Schk. (I09) Cold swamps; common.

C. teretiuscula, Gooden. (104) Norwich, Vt.; swamps.

C. torta, Boott. (37) Banks of streams; not rare.

C. tribuloides, Wahl. (127) (C. lagopodioides, Schk.) Wet grounds; many varieties.

var. cristata, Bailey. (C. cristata, Schw.) Moist grounds.

C. trisperma, Dewey. (1 22) Cold bogs; common.

C. Tuckermani, Dewey. (I3) Swamps; not uncommon.

C. umbellata, Schk. (90) Kocky hillsides.

C. utriculata, Boott. (II) Swamps ; common, and variable.

C. varia, Muhl. (86) - (C. Emmonsii, Dew.) Woods and hillsides. var. colorata, Bailey. With the type.

C. virescens, Muhl. (46) Rocky woods.

C. vulgaris, Fries. (33) Claremont, Dr. Barrows.

C. vulpinoidea, Michx. (I07) Low meadows; abundant.

Order 96. GRAMINE无. Grass Family.

Paspalum, L.

P. setaceum, Michx. Sandy fields.

Panicum, L. Panic-Grass.

P. GLABRUM, Gaudin. (Smooth Crab-Grass.) Gravelly soil. This and the next are European weeds.

P. Sanguinale, L. (Crab-Grass. Finger-Grass.) Too common. 
P. capillare, L. (Old-witch Grass.) In dry soil, everywhere.

P. virgatum, L. (Tall Panic-Grass.) River-banks, etc.; frequent.

P. xanthophysum, Gray. (Yellow Panic-Grass.) Claremont, Dr. Barrozes; Hanover and Lebanon; rare.

P. latifolium, L. (Broad-leaved Panic-Grass.) Low thickets ; frequent.

P. clandestinum, L. Moist thickets ; common.

P. depauperatum, Muhl. Dry woods; frequent.

P. dichotomum, L. Many varieties and abundant.

P. laxiflorum, Lam. Resembles the preceding, and found with it.

P. Crus-Galli, L. (Barnyard-Grass.) On waste heaps and in rich soil; very variable as to size and hairiness. From Europe.

Setaria, Beauv. Bristly Foxtail Grass.

S. verticillata, Beauv. Claremont, Dr. Barrozes; not common. All the species are European.

S. GLAUCA, Beauv. (Foxtail.) Cultivated grounds; common.

S. viridis, Beauv. (Green Foxtail.) With the preceding.

S. Italica, Kunth. (Millet. Hungarian Grass.) Rarely spontaneous.

Cenchrus, L. Bur-Grass. Hedgehog-Grass.

C. tribuloides, L. Sandy soil; the burs prickly, with sharp barbed spines.

Leersia, Swartz. White Grass.

L. Virginica, Willd. (White Grass.) Wet woods ; frequent.

L. oryzoides, Swartz. (Rice Cut-Grass.) Marshes, and common.

Andropogon, Royen. Beard-Grass.

A. furcatus, Muhl. (Blue stem.) Dry and sterile soil ; common.

A. scoparius, Michx. (Broom-grass.) Like the last, and with the same range.

Chrysopogon, Trin.

C. nutans, Benth. (Sorghum nutans, Gray.)-(Indian Grass. WoodGrass.) I)ry soil ; with the preceding species.

Phalaris, L. Canary-Grass.

P. arundinacea, L. (Reed Canary-Grass.) Wet grounds; common. var. PICTA, from Europe, is the Ribbon-Grass of gardens.

Anthoxanthum, L.

A. odoratum, L. (Sweet Vernal-Grass.) Meadows; from Europe.

Hierochloe, Gmelin. Holy-Grass.

H. borealis, Roem. \& Schultes. (Vanilla or Seneca Grass.) Hanover, etc., Flint; rare.

Aristida, L. Triple-awned Grass.

A. dichotoma, Michx. (Poverty-Grass.) Sandy soil.

Oryzopsis, Michx. Mountain Rice.

O. melanocarpa, Muhl. (Black Mountain Rice.) Rocky woods; blooming in May; common.

O. asperifolia, Michx. (White Mountain Rice.) This and the next less common than the first species. 
O. Canadensis, Torr. (Canadian Mountain Rice.) Hills and dry plains. Milium, Tourn.

M. effusum, L. (Millet-Grass.) Damp woods ; frequent.

Muhlenbergia, Schreber. Drop-seed Grass.

M. sobolifera, Trin. Rocky woods; frequent.

M. glomerata, Trin. (Spiked Muhlenbergia.) Wet banks; not rare.

M. Mexicana, Trin. (Mexican Muhlenbergia.) Common everywhere.

M. sylvatica, Torr. \& Gray. (Wood Muhlenbergia.) Rocky woods; rather scarce.

Brachyelytrum, Beauv.

B. aristatum, Beauv. Rocky woods; very common.

Phleum, L. Cat's-tail Grass.

P. PRATENSE, L. (Timothy. Herd's-Grass in New England.) A most valuable European grass.

Alopecurus, L. Foxtail Grass.

A. PRATEnsis, L. (Meadow Foxtail.) Claremont, Dr. Barrozes; moist meadows.

A. Geniculatus, L. (Floating Foxtail.) Claremont, Dr. Barrows. This and the above, European.

var. aristulatus, Torr. (A. aristulatus, Michx.) A native form found in wet places. Neither the species nor the variety is common.

Sporobolus, R. Br. Drop-seed Grass, Rush-Grass.

S. vaginæflorus, Vasey. (Vilfa vaginæflora, Torr.) Barren fields.

S. serotinus, Gray. Wet places ; common.

Agrostis, L. Bent-Grass.

A. Alba, L. (White Bent-Grass.) Cultivated from Europe.

var. vulgaris, Thurb. (Common Red-top. Herd's-Grass of Penn.)

Naturalized from Europe. The type and the variety are both good grasses, but cannot always be distinguished.

A. perennans, Tuckerm. (Thin-Grass.) In damp shade.

A. scabra, Willd. (Hair-Grass.) Dry places; common.

A. canina, L. (Brown Bent-Grass.) Meadows.

Cinna, L. Wood Reed-Grass.

C. arundinacea, L. Moist woods; frequent.

C. pendula, Trin. (C. arundinacea, var. pendula, Gray.) Wet rocks.

Calamagrostis, Adans. Reed Bent-Grass.

C. Canadensis, Beauv. (Deyeuxia Canadensis, Hook.)-(Blue-Joint Grass.) Wet grounds; a good grass.

C. Nuttalliana, Steud. (Deyeuxia Nuttalliana, Vasey.) Windsor, Vt., Leland; rare.

C. Langsdorffi, Trin. (Deyeuxia Langsdorffi, Kunth.) Moosilauke Mit.

Arrhenatherum, Beauv. Oat-Grass.

A. Avenaceum, Beauv. IVindsor, Vt., Leland; European.

Holcus, L. Meadow Soft-Grass.

H. LAnatus, L. (Velvet-Grass.) European. 
Deschampsia, Beauv. Hair-Grass.

D. flexuosa, Trin. (Aira flexuosa, L.)-(Common Hair-Grass.) Dry places.

D. cæspitosa, Beauv. (Aira cæspitosa, L.)-(Tufted Hair-Grass.) Shores of Conn. river, etc.

Avena, Tourn. Oat-Grass.

A. striata, Michx. Rocky hillsides; frequent.

[A. SAtiva, L. (Cultivated Oat.)]

Danthonia, DC. Oat-Grass. White-Grass.

D. spicata, Beauv. Dry and poor soil; hillside pastures, etc.; common. Sometimes called "June Grass," a name that belongs to Poa pratensis (Kentucky Blue-Grass).

Eatonia, Raf.

E. Pennsylvanica, Gray. Moist woods and rocks; frequent.

Eragrostis, Beauv.

E. capillaris, Nees. Sandy soil; common.

E. pectinacea, Gray. Dry, sandy fields; rare.

Dactylis, L. Orchard-Grass.

D. Glomerata, L. Very common; European.

Poa, L. Meadow-Grass. Spear-Grass.

P. Annua, L. (Low Spear-Grass.) Cultivated grounds; everywhere; naturalized from Europe.

P. COMPRESSA. L. (Wire-Grass.) Dry, sterile soil; European and very common.

P. nemoralis, L. (P. cæsia, Smith.) Thetford, Vt., Dr. Blanchard; rare.

P. serotina, Ehrh. (Fowl Meadow-Grass.) Wet meadows, and valuable.

P. pratensis, L. (June Grass. Kentucky Blue-Grass.) Common, and of value.

P. TRIVIAlis, L. (Roughish Meadow-Grass.) In wet meadows; European, and not very common.

P. alsodes, Gray. Hillside woods, Dr. Barrozus. Rare.

Glyceria, R. Br. Manna-Grass.

G. Canadensis, Trin. (Rattlesnake-Grass.) Wet places; very common.

G. elongata, Trin. IVet woods; rare.

G. nervata, Trin. Wet places; common.

G. pallida, Trin. Shallow water.

G. grandis, Watson. (G. aquatica of Am. authors; G. arundinacea, Kth.)-(Reed Meadow-Grass.) Common in marshes.

G. fluitans, R. Br. Floating in shallow water.

Festuca, L. Fescue-Grass.

F. tenella, Willd. Sterile soil; not common.

F. ovina, L. (Sheep's Fescue.) Forms tufts in meadows, and varies greatly.

F. nutans, Willd. (Nodding Fescue.) Frequent in rocky woods.

F. Elation, L. (Meadow Fescue.) A very useful grass; common in cultivation; from Europe. 
Bromus, L. Brome-Grass.

B. Kalmii, Gray. (Wild Chess.) Borders of woodlands.

B. SECAlinus, L. (Chess or Cheat.) Grain fields; a pernicious European weed, but not very common.

B. ciliatus, L. Borders of woods ; common and variable.

Lolium, L. Darne].

L. Perenne, L. (Common Darnel. Italian Rye-Grass.) Fields; a valuable European grass.

[Lolium temulentum, L. (Poison Darnel), also European, whose seeds are reputed to be poisonous, may yet be found in grain fields.]

Agropyrum, Gaertn.

A. repens, Beauv. (Triticum repens. L.)-(Couch or Witch-Grass.) Everywhere common and variable.

Elymus, L. Wild Rye.

E. Virginicus, L. River-banks; common.

E. Canadensis, L. River-banks; common.

E. striatus, Willd. Rocky woods; and like the above somewhat variable Asprella, Willd. Bottle-brush Grass.

A. Hystrix, Willd. (Gymnostichum Hystrix, Schreb.) Woods ; common.

[There belong also to this Order, Triticum vulgare (Wheat), Secale. CEREALE (Rye), Hordeun VUlgare (Barley), and ZeA Mays (Maize, Indian Corn), each including many varieties. In addition, many foreign Grasses are cultivated for ornamental purposes.] 


\section{SERIES 2. \\ CRYPTOGAMOUS OR FLOWERLESS PLANTS.}

Class III. ACROGEnS.

ORDER 97. EQUISETACEÆ. HoRsetaIl FAMILY.

Equisetum, L. Horsetail. Scouring Rush.

E. arvense, L. (Common Horsetail.) Moist, especially gravelly soil ; very common.

E. sylvaticum, L. (Wood Horsetail.) Wet shady places; common.

E. limosum, L. (Marsh Horsetail.) In shallow water; common.

E. hyemale, L. (Scouring Rush.) Wet, sandy banks; very common.

E. variegatum, Schl. Sandy banks of rivers. Hanover, N. H.; Hartford, Pomfret, and Union Village, Vt.; rare.

E. scirpoides, Michx. Wooded hillsides; common.

\section{Order 98. FILICES. FERNS.}

Polypodium, L. Polypody.

P. vulgare, L. On rocks in woods; common.

Adiantum, L. Maidenhair Fern.

A. pedatum, L. Rich, moist woods; common.

Pteris, L. Brake.

P. aquilina, L. (Common Brake.) Pastures and woods; everywhere.

Pellæa, Link. Cliff-Brake.

P. gracilis, Hook. Wet, limestone rocks. Norwich, Vt., Dr. Edward Hyde; rare.

Asplenium, L. Spleenwort.

A. Trichomanes, L. Shaded rocks; common.

A. ebeneum, Ait. Rocky woods; common.

A. angustifolium. Michx. Rich, moist woods. Hanover, N. H., Miss Hitchcock; Windsor and Pomfret, Vt. Not very abundant.

A. thelypteroides, Michx. Rich woods; common.

A. Filix-fœmina, Bernh. (Lady-Fern.) Frequent and variable. 
Camptosorus, Link. Walking-Leaf.

C. rhizophyllus, Link. On shaded rocks; very local. Lebanon, N. H., Dr. Barrozes; Windsor, Vt., Leland; Norwich, Vt., Miss Loveland.

Phegopteris, Fée. Beech Fern.

P. polypodioides, Fée. Damp woods; common.

P. hexagonoptera, Fée. Norwich, Vt., F. H. Herrick; scarce.

P. Dryopteris, Fée. Rocky woods; common.

Aspidium, Swartz. Shield Fern.

A. Thelypteris, Swartz. Marshes; abundant.

A. Noveboracense, Swartz. Wet woods; abundant.

A. spinulosum, Swartz. Damp woods. This is the European type, and is rare.

var. intermedium, Gray. Common.

var. dilatatum, Gray. Summit of Mt. Ascutney, Vt., Leland; Killington Mt., Denslow.

A. Boottii, Tuckerm. (A. spinulosum, var., Boottii, Man., 5th ed.) Not rare in damp woods.

A. cristatum, Swartz. Sivamps; common.

var. Clintonianum, D. C. Eaton. Hanover, N. H., Miss Hitchcock; and probably not very rare.

A. Goldianum, Hook. Rich and moist woods; Hanover, N. H., Miss Hitchcock; not abundant.

A. marginale, Swartz. Rocky hillsides, etc. ; abundant.

A. acrostichoides, Swartz. (Christmas Fern.) Woods; common and variable.

A. aculeatum, Swartz., var. Braunii, Koch. North Pomfret and Killington Mt., Vt., Morgan. "The Gulf" in Weathersfield, Vt., Leland; rare.

Cystopteris, Bernh. Bladder Fern.

C. fragilis, Bernh. Shaded ravines; very common.

C. bulbifera, Bernh. Moist cliffs and rich woods; frequent.

\section{Onoclea, L.}

O. sensibilis, L. (Sensitive Fern.) Very common.

O. Struthiopteris, Hoff. (Struthiopteris Germanica,Willd.)-(Ostrich Fern.) Rich alluvial soil ; abundant.

Woodsia, R. Br.

W. Ilvensis, R. Br. On rocks; common.

W. obtusa, Torrey. Rocky banks; rather rare.

Dicksonia, L'Her.

D. pilosiuscula, Willd. (I). punctilobula, Kunze.) Common in moist, shady places and pastures.

Osmunda, L. Flowering Fern.

O. regalis, L. (Queen-Fern. Royal-Fern.) Wet woods; rather common.

O. Claytoniana, L. (Clayton's Fern.) Wet woods; very common.

O. cinnamomea, L. (Cinnamon Fern.) Wet woods; common. 
Order 99. OPHIOGLOSSACE开. AdDER's-Tongue Family.

Botrychium, Swartz. Grape-Fern. Moonwort.

B. simplex, Hitch. Hillside pastures; very seldom met with.

B. matricariæfolium, Al. Br. Not common.

B. lanceolatum, Angs. Hillside pastures with the preceding two species, and like them very rarely collected.

B. ternatum, Swartz. Pastures and hillsides. Among the numerous varieties described by Prof. D. C. Eaton, the more common are:var. intermedium. A large form.

var. obliquum. Medium in size and more common.

var. dissectum. Occasionally with the last. See Eaton's Ferns of North America.

B. Virginianum, Swartz. Rich woods; common.

Ophioglossum, L. Adder's-Tongue.

O. vulgatum, L. Bogs and pastures; not uncommon.

Order roo. LYCOPODIACE开. Club-Moss Family.

Lycopodium, L. Club-Moss.

L. lucidulum, Michx. Damp woods ; common.

L. inundatum, L. Clayey ground; Grantham Mt., Plymouth, and Hanover, N. H.; Hartland, Vt. ; etc.; scarce.

L. annotinum, L. Woods; common.

L. obscurum, L. Seldom distinguished from the variety. var. dendroideum. (L. dendroideum, Michx.)-(Tree Club-Moss.)

Woods; common.

L. clavatum, L. (Common Club-Moss.) Dry woods; abundant.

L. complanatum, L. (Creeping Ground-Pine.) With the above, common. var. chamæcyparissus, Eaton. Occasional.

Order ioi. SELAGINELLACE涩. Selaginella Family.

Selaginella, Beauv.

S. rupestris, Spring. Dry rocks; common.

S. apus, Spring. Wet places ; about springs ; much less common.

Isoetes, L. Quillwort.

I. echinospora, Durieu. The type form is European, but the following varieties are met with :

var. Braunii, Engelm. Margin of ponds and streams.

var. muricata, Engelm. Mouth of Pompanoosuc river, Norwich, Vt.

var. Boottii, Engelm. Goose Pond, Canaan; Conn. river, Hanover.

I. riparia, Engelm. Margin of Conn. river, Hanover.

[I. lacustris, L. Assigned to "Lebanon" in the Geological Survey of New Hampshire. The species referred to is probably some var. of $I$. echinospora; but if $I$. lacustris is a New England species, it should be found within our limits. Apparently very little is known about it.]

[All the members of this genus are of somewhat uncertain determination. They are found in our springs and ponds, and in the still and shallow margins of our streams, or during a period of drought may be entirely out of the water.] 


\title{
LIST OF VERTEBRATES. ${ }^{1}$
}

\author{
(BY PROF. T. W. D. WORTHEN.)
}

\section{CLASS I. PISCES. THE FISHES.}

\section{ORDER I. NEMATOGNATHI.}

Family i. SILURID开. The Cat-fishes.

Ameiurus, Rafinesque.

A. nebulosus (Le Sueur). Common Bullhead. Horn Pout. Common.

\section{ORDER II. EVENTOGNATHI.}

FAMIly 2. CATOSTOMID无. The Suckers.

Catostomus, Le Sueur. Fine-scaled Suckers.

C. teres (Mitchill). Common Sucker. White Sucker. Abundant.

C. nigricans, Le Sueur. Hog-Sucker. Stone Roller. Common.

Family 3. CYPRINID五. The Minnows.

Notropis, Rafinesque. American Minnows.

N. bifrenatus (Cope). Mud-dace. Brook Minnow. Common.

N. Whipplei (Girard). Silver-Fin. River-Shiner. Not rare.

N. megalops (Rafinesque). Common Shiner. Red-Fin. Dace. Common. Rhinichthys, Agassiz.

R. atronasus (Mitchill). Black-nosed Dace. "Red-Fin." Common.

Semotilus, Rafinesque.

S. bullaris (Rafinesque). Fall Fish. Chub. Dace. Roach. Abundant. Notemigonus, Rafinesque.

N. chrysoleucus (Mitchill). Golden Shiner. Shiner. Bream. Abundant.

1 In this list are included, so far as known, all Animals belonging to the Sub-Kingdom VerteBrata, found within about thirty miles of Hanover, $N . H$. The classification, including citation of authorities, is that given in the Manual of the Vertebrate Animals of the Northern United States by Pres. David S. Fordan, Fifth edition, rewritten and enlarged, 1888 , where full descriptions may be found. 
Order iII. isospondyli. The Salmon, Herring, etc.

Family 4. SALmonid瓜. The Salmon.

Salvelinus (Nilsson), Richạrdson.

S. namaycush (Walbaum). Lake Trout. Mackinaw Trout. Salmon Trout. Longe. Rare. M. V. B. Knox.

S. fontinalis (Mitchill). Brook Trout. Speckled Trout. Common.

S. aureolus, Bean. Sunapee Lake Trout. In Sunapee Lake. Not rare.

Order IV. HAPlomi. The Pike-like Fishes.

Family 5. ESOCID卌. The Pikes.

Esox (Artedi), Linnæus.

E. Americanus, Gmelin. Grass Pickerel. Common.

E. reticulatus, Le Sueur. Pickerel. Eastern Pickerel. Abundant.

E. lucius, L. Pike. Northern Pickerel. Common.

Order V. Apodes. The Eels.

Family 6. ANGUILlid仺. The True Eels.

Anguilla, Thunberg.

A. anguilla (L.). Eel. Abundant.

Order Vi. acanthopteri. The Spiny-rayed Fishes.

Family 7. CENTRARChID咂. The Sun-fishes.

Lepomis, Rafinesque. Sun-fishes.

L. auritus (Linnæus). Long-eared Sun-fish. Sun-Perch. Common.

L. gibbosus (L.). Common Sun-fish. Bream. Pumpkin-seed. Abundant. Micropterus, Lacépède. Black Bass.

M. dolomieu, Lacépède. Small-mouthed Black Bass. Abundant.

Family 8. PERCID君. The Perches.

Etheostoma, Rafinesque. Darters.

E. Olmstedi, Storer. Darter. Spotted Darter. Common.

Perca (Artedi), Linnæus.

P. flavescens (Mitchill). Yellow-Perch. Perch. Abundant.

Stizostedion, Rafinesque.

S. vitreum (Mitchill). Wall-eye. Pike Perch. Plymouth, Vt.

Family 9. COTTID床. The Sculpins.

Cottus (Artedi) Linnæus. Miller's Thumb.

C. gracilis, Heckel. Common.

Family io. GADID无. The Cod-Fishes.

Lota, Cuvier. Burbot. Chub Eel. Ling.

L. Iota (L.), var. maculosa, Le Seur. Eel Pout. Common. 


\section{CLASS II. BATRACHIA. THE BATRACHIANS.}

Order Vil. URodela. The Salamanders.

Fanily it. AMBLYSTOMATID不. The Blunt-nosed SalamanDERS.

Amblystoma, Tschudi.

A. punctatum (L.). Spotted Salamander. Common.

A. tigrinum (Green). Not rare.

\section{FAMILY I2. PLETHODONTID无.}

Plethodon, Tschudi.

P. erythronotus (Green). Common.

P. glutinosus (Green). Not rare.

Spelerpes, Rafinesque.

S. bilineatus (Green). Not rare.

S. longicauda (Green). Cave Salamander. Rare.

S. ruber (Daudin). Common.

Family 13. PLEURODELIDÆ. The Newts.

Diemyctylus, Rafinesque.

D. viridescens, Rafinesque. Newt. Evet. Eft. Abundant. var. miniatus, Rafinesque. Red Eft. Common.

Order Vili. SAlientia. The Tailless Batrachians.

Family i4. BUFONID无. The Toads.

Bufo, Laurenti.

B. lentiginosus, Shaw. American Toad. Abundant.

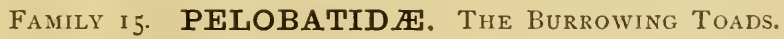

Scaphiopus, Holbrook.

S. Holbrooki, Harlan. Spade-foot. Probable.

Family 16. HYLID杘. The Tree Frogs.

Acris, Dumeril \& Bibron.

A. gryllus, Le Conte. Cricket Frog. Probable. 
Chorophilus, Baird.

C. triseriatus (Wied). Swamp Tree Frog. Probable.

Hyla, Laurenti.

H. versicolor, Le Conte. Common Tree Toad. Common.

H. Pickeringii, Holbrook. Peeping Frog. Common.

Family 17. RANID无. The Frogs.

Rana, Linnæus.

R. virescens, Kalm. Common Frog. Leopard Frog. Abundant.

R. palustris, Le Conte. Pickerel Frog. Rather common.

R. sylvatica, Le Conte. Wood Frog. Rather common.

R. clamata, Daudin. Green Frog. Common.

R. Catesbiana, Shaw. Bull Frog. Not rare. 


\section{CLASS III. REPTILIA. THE REPTILES.}

Order IX. OPHidia. The Serpents.

Fanily is. COLUBRID开. The Colubine SNakes.

Storeria, Baird \& Girard.

S. occipitomaculata (Storer). Red-Bellied Snake. Common.

Eutainia, Baird \& Girard. Garter Snakes.

E. saurita (L.). Riband Snake. Probable.

E. sirtalis (L.). Common Garter Snake. Striped Snake. Abundant. var. ordinata (L.). Common.

var. dorsalis (Baird \& Girard). Common.

Tropidonotus, Kuhl.

T. sipedon (L.). Water Snake. "Moccasin." Rather rare.

Liopeltis (Fitzinger), Cope.

L. vernalis (De Kay). Grass Snake. Green Snake. Rather common.

Diadophis, Baird \& Girard.

D. punctatus (L.). Ring-necked Snake. Not common.

Ophibolus, Baird \& Girard.

(). doliatus (L.), var. triangulus (Boie). Milk Snake. Adder. Spotted Adder. Rather common.

Heterodon, Beauvais.

H. platyrhinus, Latreille. Spreading Adder. Adder. Blowing Viper. Rather common.

Family 19. CROTALID无. The Rattlesnakes.

Crotalus, Linnæus.

C. horridus, L. Common Rattlesnake. Not rare; Springfield, Vt., and vicinity, C. D. Hull.

Order X. TESTUdinAtA. The Turtles.

Family 20. ChELYDRID压. The Snapping Turtles.

Chelydra, Schweigger.

C. serpentina (L.). Common Snapping Turtle. Abundant. 
64 LIST OF VETEBRATES.

Family 21. EMYDid仺. The Pond Turtles.

Chrysemys, Gray.

C. picta (Hermann). Painted Turtle. Mud Turtle. Common. Chelopus, Rafinesque.

C. insculptus (Le Conte). Wood Tortoise. Not common.

C. guttatus (Schneider). Speckled Tortoise. Probable. 


\section{CLASS IV. AVES. THE BIRDS.}

\section{Order Xi. PYGOPODES. The Diving Birds.}

\section{FAMILY 22. PODICIPID无. ThE GREBEs.}

Podiceps, Latham.

P. cristatus, Latham. Crested Grebe. Rare migrant. C. H. Storrs.

Colymbus, Linnæus.

C. Holbölli (Reinhardt). Red-necked Grebe. Migrant. C. A. Dowuns.

C. auritus, L. Horned Grebe. Migrant. C. A. Dowuns.

Podilymbus, Lesson.

P. podiceps (L.). Diedapper. Dab-chick. Dipper Duck. Migrant.

FAMILY 23. URINATORID王. THE LOONS.

Urinator, Cuvier.

U. imber (Gunner). Common Loon. Diver. Northern Diver. Rather common summer resident.

\section{FAMILY 24. ALCID正. The AUKS.}

Alle, Link.

A. alle (L.). Dovekie. Sea Dove. Little Auk. Rare visitor. $F$. $H$. Herrick.

Order XII. LONGIPENNES. The Long-Winged Swlmers.

$$
\text { FAMily 25. LARID正. The Gulls. }
$$

Larus, Linnæus.

L. argentatus, Brünnich. Herring Gull. Common Gull. Not common summer visitor.

L. Philadelphia, Ord. Bonaparte's Gull. Rare summer visitor.

Sterna, Linnæus.

S. paradisæa, Brünich. Arctic Tern. Occasional summer visitor. $M . V$. B. K'nox.

S. Antillarum, Lesson. Least Tern. Rare summer visitor.

Family 26. PHALACROCORACID王: The Cormorants.

Phalacrocorax, Brisson.

P. carbo (L.). Common Cormorant. Very rare visitor. C. A. Dowuns. 


\section{Order XIII. ANSERES. The Ducks and Geese. \\ FAMily 27. ANATID无. The DuCks and Geese.}

Merganser, Brisson.

M. Americanus (Cassin). Merganser. Goosander. Not rare migrant.

Lophodytes, Reichenbach.

L. cucullatus (L.). Hooded Merganser. Sheldrake. Occasional visitor. M. V. B. Knox.

Anas, Linnæus.

A. boschas, L. Mallard Duck. Tame Duck. "Original of the common domesticated duck." Rather rare migrant.

A. obscura, Gmelin. Black Duck. Common migrant. Occasional summer resident.

A. discors, L. Blue-winged Teal. Migrant.

A. Carolinensis, Gmelin. Green-winged Teal. Migrant.

Aix, Boie.

A. sponsa (L.). Wood Duck. Summer Duck. Not rare summer resident.

Erismatura, Bonaparte.

E. rubida (Wilson). Ruddy Duck. Common migrant.

Branta, Scopoli.

B. Canadensis (L.). Wild Goose. Canada Goose. Rather common migrant.

Order XiV. Herodiones. The Herons and Storks.

Family 28. ARDEID雇. The Herons.

Botaurus, Stephens.

B. lentiginosus (Montagu). Bittern. Indian Hen. Common summer resident.

B. exilis (Gmelin). Least Bittern. Not rare summer resident. C. $H$. Storrs.

Ardea, Linnæus.

A. herodias, L. "Great Blue Heron. Rather common summer resident.

A. virescens (L.). Green Heron. Rather common summer resident.

Nycticorax, Stephens.

N. nycticorax (L.). Night-heron. Qua Bird. Squawk. Rather rare summer resident.

Order XV. PAlUdicole. The Cranes and Rails.

Family 29. RALLID咂. The Rails.

Rallus, Linnæus.

R. Virginianus, L. Virginia Rail. Probable summer resident. 
Porzana, Vieillot.

P. Carolina (L.). Carolina Rail. Sora. Occasional summer resident. R. Chellis.

Fulica, Linnæus.

F. Americana (Gmelin). Coot. Mud Hen. Rather rare summer resident.

Order XVI. LIMICOLÆ. The Shore-birds.

Fanily 30. SCOLOPACID无. The Snipe.

Philohela, Gray.

P. minor (Gmelin). American Woodcock. Common migrant; not rare summer resident.

Gallinago, Leach.

G. delicata (Ord.). Wilson's Snipe. Not rare summer resident. C. $H$. Storrs; R. Chellis.

Tringa, Linnæus.

T. nıaritima, Brünnich. Purple Sandpiper. Not common summer risitor.

T. minutilla, Vieillot. Least Sandpiper. Peep. Rare summer visitor. C. H. Storrs.

Totanus, Bechstein.

T. melanoleucus (Gmelin). Greater Tell-tale. Yellow Shanks. Occasional summer visitor. C. H. Storrs; R. Chellis.

T. flavipes (Gmelin). Lesser Tattler. Yellow Legs. Not common summer visitor.

Bartramia, Lesson.

B. Inngicauda (Bechstein). Upland Sandpiper. Rather common summer resident.

Actitis, Boie.

A. macularia (L.). Tip-up. Teeter-Tail. Spotted Sandpiper. Common summer resident.

\section{Family 31. CHARADRIID卌. The Plovers.}

屁gialitis, Boie.

A. vocifera (L.). Kildeer. Rare summer visitor. B. T. Blanpied.

Æ. meloda (Ord.). Piping Plover. Occasional summer resident. C. H. Storrs.

Arenaria, Brisson.

A. interpres (L.). Turnstone. Very rare sumnier visitor. C. A. Downs.

Order XVII. GallinÆ. The Gallinaceous Birds.

FAMILy 32. TETRAONID虚. The Grouse.

Colinus, Lesson.

C. Virginianus (L.). Bob-IVhite. Quail. Rare summer visitor or resident. T. H. Chubb; B. T. Blanfied. 
Dendragapus, Elliott.

D. Canadensis (L.). Spruce Partridge. Canada Grouse. Very rare resident.

Bonasa, Stephens.

B. umbellus (L.). Ruffed Grouse. Partridge. Common resident.

Order XViII. COlUMBæ. The Doves.

Fanily 33. COLUMbid咂. The Pigeons.

Ectopistes, Swainson.

E. migratorius (L.). Wild Pigeon. Passenger Pigeon. Rather rare summer resident.

Zenaidura, Bonaparte.

Z. macroura (L.). Mourning Dove. Turtle Dove. Carolina Dove. Very rare summer resident.

\section{Order Xix. RAPtores. The Birds of Prey.}

\section{Family 34. FALCONID君. The Falcons.}

Circus, Lacépède.

C. Hudsonius (L.). Marsh Harrier. Common summer resident.

Accipiter, Brisson.

A. velox (Wilson). Sharp-Shinned Hawk. "Pigeon Hawk." Rather common summer resident.

A. Cooperi, Bonaparte. Chicken Hawk. Common summer resident.

A. atricapillus (Wilson). Goshawk. Rather rare winter visitor and rare resident.

Buteo, Cuvier.

B. borealis (Gmelin). Hen Hawk. Red-Tailed Hawk. Common resident.

B. latissimus (Wilson). Broad-Winged Hawk. Not rare summer resident.

B. lineatus (Gmelin). Chicken Hawk. Red-Shouldered Hawk. Rather common resident.

Archibuteo, Brehm.

A. lagopus (Brünnich). Rough-Legged Hawk. Black Hawk. Probable resident.

Aquila, Brisson.

A. chrysaëtos (L.). Golden Eagle. Probable summer resident. Kñox.

Haliaëtus, Savigny.

H. leucocephalus (L.). Bald Eagle. Rather rare summer resident.

Falco, Linnæus. Falcons.

F. rusticolus (L.). Gray Gyrfalcon. Jerfalcon. Probable winter visitor.

F. peregrinus, Tunstall. Peregrine Falcon. Duck Hawk. Not common resident. 
F. columbarius, L. Pigeon Hawk. American Merlin. Rather common summer resident and rare resident.

F. sparverius, L. Sparrow Hawk. Rusty Crowned Falcon. Rather common summer resident.

Pandion, Savigny.

P. haliaëtus (L.). Osprey. Fish Hawk. Rather common summer resident.

\section{FAMILY 35. BUBONID无. The Owls.}

Asio, Brisson.

A. Wilsonianus (Lesson). Long-eared Owl. Rare resident. T. Bakes.

A. accipitrinus (Pallas). Short-eared Owl. Very rare resident.

Syrnium, Savigny.

S. nebulosum (Forster). Barred Owl. Rather common resident.

Scotiaptex, Swainson.

S. cinereum (Gmelin). Great Gray Owl. Spectral Owl. Probable winter visitor.

Nyctala, Brehm.

N. Tengmalmii (Gmelin). Sparrow Owl. Probable winter visitor.

N. Acadica (Gmelin). Saw-whet Owl. Acadian Owl. Rather rare resident.

Megascops, Kaup.

M. asio (L.). Screech Owl. Red Owl. Rather common resident.

Bubo, Duméril.

B. Virginianus (Gmelin). Great Horned Owl. Not rare resident.

Nyctea, Stephens.

N. nyctea (L.). Snowy Owl. Very rare winter visitor.

Surnia, Duméril.

S. ubula (L..). Hawk Owl. Day Owl. Rare winter visitor or resident.

Order yX. COCCYGES. The Cuckoo-like Birds.

FAmily 35. CUCULID无, The Cuckoos.

Coccyzus, Vieillot.

C. Americanus (L.). Yellow-billed Cuckoo. "Rain Crow." Rather common summer resident,

C. erythrophthalmus (Wilson). Black-billed Cuckoo. Common summer resident.

FAMILY 37. ALCEDINID无. The Kingfishers.

Ceryle, Boie.

C. alcyon (L.). Belted Kingfisher. Common summer resident. 
Order XXi. PiCi. The Woodpeckers and Wrynecks. Family 38. PICID开. The Woodpeckers.

Dryobates, Boie.

D. villosus (L.). Hairy Woodpecker. Big Sap-Sucker. Common winter visitor; not rare resident.

D. pubescens (L.). Downy Woodpecker. Little Sap-Sucker. Common resident.

Picoides, Lacépède.

P. arcticus (Swainson). Black-backed Woodpecker. Not common winter resident.

P. Americanus, Brehm. Striped Woodpecker. Not rare winter visitor.

Sphyrapicus, Baird.

S. varius (L.). Yellow-bellied Woodpecker. Common summer resident.

Ceophlœus, Cabanis.

C. pileatus (L.). Logcock. Big Black Woodpecker. Pileated Woodpecker. Not rare resident.

Melanerpes, Swainson.

M. erythrocephalus (L.). Red-headed Woodpecker. Rather rare summer resident.

M. Carolinus (L..). Red-bellied Woodpecker. Possible summer resident. Colaptes, Swainson.

C. auratus (L.). Yellow-Hammer. Flicker. Wood-wall. Golden-winged Woodpecker. High-Holer. Common summer resident.

Order XXII. MACROCHIRES. The Siwifts and Humming Birds.

Family 39. CAPRIMULGid乍. The Goatsuckers.

Antrostomus, Gould.

A. vociferus (Wilson). Whippoorwill. Night Jar. Common summer resident.

Chordeiles, Swainson.

C. Virginianus (Gemlin). Night Hawk. Bull Bat. Very common summer resident.

FAMIly 40. MICROPODID无. ThE SwifTs.

Chætura, Stephens.

C. pelagica (L.). Chimney Swift. Chimney Swallow. Abundant summer resident.

Family 4i. TROChilid不. The Humming Birds.

Trochilus, Linnæus.

T. colubris, L. Ruby-throated Humming Bird. Common summer resident. 
Order XXIII. PASSERES. The PAsserine Birds.

FAMILY 42. TYRANNID尼. THE FlyCATChERS.

Tyrannus, Cuvier.

T. tyrannus (L.). King Bird. Bee Martin. Common summer resident. Myiarchus, Cabanis.

M. crinitus (L.). Great Crested Flycatcher. Not rare summer resident.

Sayornis, Bonaparte.

S. Phœbe (Latham). Pewee. Phœbe. Abundant summer resident.

Contopus, Cabanis.

C. borealis (Swainson). Olive-sided Flycatcher. Rather rare summer resident.

C. virens (L.). Wood Pewee. Common summer resident.

Empidonax, Cabanis.

E. flaviventris, Baird. Yellow-bellied Flycatcher. Not common summer resident.

E. Acadiacus (Gmelin). Small Green-crested Flycatcher. Not rare summer resident.

E. pusillus (Swainson), var. Trailli, Audubon. Traill's Flycatcher. Not common summer resident.

E. minimus, Baird. Least Flycatcher. Very common summer resident.

\section{Family 43. ALAUDID无. The LARKs.}

Otocoris, Bonaparte.

O. alpestris (Forster). Shore Lark. Horned Lark. Not rare migrant. G. D. Hull.

Family 44. CORVID虚. The Crows and Jays.

Cyanocitta, Strickland.

C. cristata (L.). Blue Jay. Common resident.

Perisoreus, Bonaparte.

P. Canadensis (L.). Canada Jay. Gray Jay. Whiskey Jack. Occasional visitor; possible resident. Storrs.

Corvus, Linnæus.

C. Americanus, Audubon. Crow. Very common resident.

Family 45. ICTERID画. The American “Orioles" and "BlackBIRDS."

Dolichonyx, Swainson.

D. oryzivorus (L.). Bobolink. Reed Bird. Rice Bird. Very common summer resident.

Molothrus, Swainson.

M. ater (Boddaert). Cow Blackbird. Cowbird. Common summer resident. 
Agelaius, Vieillot.

A. phœniceus (L.). Red-winged Blackbird. Swamp Blackbird. Common summer resident.

Sturnella, Vieillot.

S. magna (L.). Meadowlark. Not rare summer resident.

Icterus, Brisson.

I. galbula (L.). Baltimore Oriole. Golden Robin. Fire Bird. Common summer resident.

Scolecophagus, Swainson.

S. Carolinus (Müller). Rusty Grackle. Rusty Blackbird. Not rare migrant.

\section{Quiscalus, Vieillot.}

Q. quiscula (L.). Crow Blackbird. Purple Grackle. Rather common summer resident.

FAMILY 46. FRINGILLID开. ThE Finches.

Pinicola, Vieillot.

P. enucleator (L.). Pine Grosbeak. Rather common winter resident.

Carpodacus, Kaup.

C. purpureus (Gmelin). Purple Finch. Very common summer resident.

Loxia, Linnæus.

L. leucoptera, Gmelin. White Winged Crossbill. Rather rare winter visitor.

L. curvirostra (L.). Red Crossbill. Not rare winter resident; rare resident.

Acanthis, Bechstein.

A. linarius (L.). Red Poll Linnet. Rather common winter resident.

Spinus, Boie.

S. tristis (L.). Yellow Bird. Thistle Bird. American Goldfinch. Abundant resident.

S. pinus (Wilson). Pine Siskin. Pine Finch. Rather common winter visitor; not rare resident.

Plectrophenax, Stejneger.

P. nivalis (L.). Snow Bunting. Very common winter resident.

Calcarius, Bechstein.

C. Lapponicus (L.). Lapland Longspur. Rare winter visitor.

Poocætes, Baird.

P. gramineus (Gmelin). Bay-winged Bunting. Grass Sparrow. Ground Bird. Abundant summer resident.

Passer, Brisson.

P. domesticus (L.). English Sparrow. Abundant resident.

Ammodramus, Swainson.

A. Sandwichensis (Gmelin), var. Savanna. Savanna Sparrow. Not common summer resident. 
A. savannarum (Gmelin). Grasshopper Sparrow. Probable summer resident.

Zonotrichià, Swainson.

Z. leucophrys (Forster). White-crowned Sparrow. Common migrant.

Z. albicollis (Gmelin). White-throated Sparrow. Peabody Bird. Rather common resident.

Spizella, Bonaparte.

S. monticola (Gmelin). Tree Sparrow. Rather rare winter visitor.

S. socialis (Wilson). Chippy. Chipping Sparrow. Abundant summer resident.

S. pusilla (Wilson). Field Sparrow. Common summer resident.

Junco, Wagler.

J. hyemalis (L.). Snow Bird. Common migrant; not rare resident.

Melospiza, Baird.

M. fasciata (Gmelin). Song Sparrow. Very common summer resident.

M. Georgiana (Latham). Swamp Sparrow. Rather common summer resident.

Passerella, Swainson.

P. iliaca (Merrem). Fox Sparrow. Common migrant.

Pipilo, Vieillot.

P. erythrophthalmus (L.) Chewink. Marsh Robin. Towhee. Not rare summer resident.

Habia, Reichenbach.

H. Ludoviciana (L.). Rose-breasted Grosbeak. Rather common summer resident.

Passerina, Vieillot.

P. cyanea (L.). Indigo Bird. Very common summer resident.

\section{Family 47. TANAGRID无. The Tanagers.}

Piranga, Vieillot.

P. erythromelas, Vieillot. Scarlet Tanager. Rather common summer resident.

Family 48. HIRUNDINID卌. The Swallows.

Progne, Boie.

P. subis (L.). Purple Martin. Rather common summer resident.

Petrochelidon, Cabanis.

P. lunifrons (Say). Cliff Swallow. Abundant summer resident.

Chelidon, Forster.

C. erythrogaster (Boddaert). Barn Sivallow. Abundant summer resident. Tachycineta, Cabanis.

T. bicolor (Vieillot). IVhite-bellied Swallow. Very common summer resident. 
Clivicola, Forster.

C. riparia, L. Bank Swallow. Sand Nartin. Very common summer resident.

\section{Family 49. AMPELID无. The Chatterers.}

Ampelis, Linnæus.

A. garrulus. L. Bohemian Wax Wing. Northern Wax Wing. Probable winter visitor.

A. cedrorum (Vieillot). Cedar Bird. Cherry Bird. Very common summer resident.

\section{Family 50. LANIID开。 The Shrikes.}

Lanius, Linnæus.

L. borealis, Vieillot. Great Northern Shrike. Butcher-bird. Rather common winter resident, and rather rare resident.

L. Ludovicianus, L. Logger-head Shrike. Rather rare resident.

FAMILy 5i. VIREONID压. The Vireos.

Vireo, Vieillot.

V. olivaceus (L.). Red-eyed Vireo. Greenlet. Very common summer resident.

V. gilvus (Vieillot). Warbling Vireo. Rather common summer resident.

V. flavifrons, Vieillot. Yellow-throated Vireo. Not rare summer resident.

V. solitarius (Wilson). Blue-headed Vireo. Rather rare summer resident.

V. Noveboracensis (Gmelin). White-eyed Vireo. Rather common summer resident.

FAMily 52. MNIOTILTID君. The New World Warblers.

Mniotilta, Vieillot.

M. varia (L.). Black and White Creeper. Rather common summer resident.

Helminthophila, Ridgway.

H. chrysoptera (L.) Golden-winged IVarbler. Probable migrant and resident.

H. pinus (L.). Blue-winged Yellow Varbler. Possible summer resident.

H. peregrina (Wilson). Tennessee Warbler. Rare migrant, and possible resident.

H. celata (Say). Orange-crowned Warbler. Rare summer resident. $F . H$. Herrick.

H. ruficapilla (Wilson). Nashville Warbler. Not common summer resident. 
Compsothlypis, Cabanis.

C. Americana (L.). Blue Y'ellow-backed Warbler. Not common summer resident.

Dendroica, Gray.

D. æstiva (Gmelin). Summer Warbler. Golden Warbler. Rather common summer resident.

D. cærulescens (L.). Black-throated Blue Warbler. Rather rare summer resident. C. H. Storrs.

D. coronata (L.) Yellow-rumped Warbler. Common migrant and probable resident.

D. maculosa (Gmelin). Black and Yellow Warbler. Not rare summer resident.

D. Pennsylvanica (L.). Chestnut-sided Warbler. Rather common summer resident.

D. castanea (Wilson). Bay-breasted Warbler. Autumn Warbler. Rare summer resident.

D. striata (Forster). Black-poll Warbler. Common migrant.

D. Blackburnix (Gmelin). Orange-throated Warbler. Blackburnian Warbler. Rare summer resident.

D. virens (Gmelin). Black-throated Green Warbler. Rather common summer resident.

D. vigorsi (Audubon). Pine-creeping Warbler. Common migrant, and rather rare summer resident.

D. discolor (Vieillot). Prairie Warbler. Probable summer resident.

D. palnarum (Gmelin). Red-poll Warbler. Rather common migrant.

Seiurus, Swainson.

S. aurocapillus (L.). Oven-bird. Golden-crowned "Thrush." Rather common summer resident.

S. Noveboracensis (Gmelin). Water Wagtail. Water Thrush. Probable summer resident.

Geothlypis, Cabanis.

G. Philadelphia (Wilson). Mourning Warbler. Rare summer resident.

G. trichas (L.) Maryland Yellow Throat. Common summer res dent.

Sylvania, Nuttall.

S. pusilla (Wilson). Green Black-capped Warbler. Kather common migrant.

S. Canadensis (L.). Canada Warbler. Not common summer resident. C. H. Storrs.

Setophaga, Swainson.

S. ruticilla (L.). American Redstart. Rather common summer resident.

Family 53. MOTACILLID无. The inagtails.

Anthus, Bechstein.

A. Pennsylvanicus (Latham). Brown Lark. Tit-lark. Rather common migrant. 
Family 54. TROGLODYTID丑. The Wrens and Mocking-

Mimus, Boie. BIRDS.

M. polyglottos (L.). Mocking-Bird. Very rare visitor. C. A. Dowens.

Galeoscoptes, Cabanis.

G. Carolinensis (L.). Cat-Bird. Very common summer resident.

Harporhynchus, Cabanis.

H. rufus (L.). Brown Thrush. Brown Thrasher. Rather common summer resident.

Troglodytes, Vieillot.

T. aedon, Vieillot. House Wren. Common summer resident.

T. hiemalis, Vieillot. Winter Wren. Not rare migrant.

Cistothorus, Cabanis.

C. stellaris (Lichtenstein). Short-billed Marsh Wren. Probable summer resident.

C. palustris (Wilson). Long-billed Marsh Wren. Possible summer resident.

\section{Family 55. CERThiID开. The Creepers.}

Certhia, Linnæus.

C. familiaris, L. Brown Creeper. Rather rare summer resident.

Family 56. PaRID不. The Nuthatches and Titmice.

Sitta, Linnæus.

S. Carolinensis, Latham. White-bellied Nuthatch. "Sap-sucker." Coinmon resident.

S. Canadensis, L. Red-bellied Nuthatch. Common winter resident, and rather rare resident.

Parus, Linnæus.

P. atricapillus, L. Black-capped Titmouse. Chickadee. Very common resident.

FAMILY 57. SYLVIID开. The Old World Warblers.

Regulus, Cuvier.

R. satrapa. Lichtenstein. Golden-crowned Kinglet. Common migrant, and not rare winter resident.

R. calendula (L.). Ruby-crowned Kinglet. Common migrant.

Family 58. TURDID虚. The Thrushes.

Turdus, Linnæus.

T. mustelinus, Gmelin. Wood Thrush. Rare summer resident.

T. fuscescens, Stephens. Tawny Thrush. Wilson's Thrush. Not common summer resident.

T. ustulatus, Nuttall. Olive-backed Thrush. Rare summer resident. 
T. Aonalaschkæ, Gmelin, var. Pallasi, Cabanis. Hermit Thrush. Common summer resident.

Merula, Leach.

M. migratoria (L.). Robin. American Red Breast. Abundant summer resident, and very rare resident.

Sialia, Swainson.

S. sialis (L.). Common Blue Bird. Very common summer resident. 


\section{CLASS V. MAMMALIA. THE MAMMALS.}

Order XXIV. GLIRES. The Rodents or GNawers.

FAMily 59. LEPORID无. The Hares.

Lepus, Linnæus.

L. Americanus, Erxleben. White Rabbit. Northern Hare. Common.

Family 6o. HYSTRICID无. The Porcupines.

Erethizon, Frédéric Cuvier.

E. dorsatus (I..). Canada Porcupine. Not rare. This is wrongly called "Hedgehog", which is European.

Family 6i. ZapoDid在. The Jumping Mice.

Zapus, Coues.

Z. Hudsonius (Zimmerman). Jumping Mouse. Rare.

FAMily 62. MURID开. The MiCE.

Fiber, Cuvier.

F. zibethicus (L.). Muskrat. Common.

Arvicola, Lacépède: Field Mice.

A. Pennsylvanicus, Ord. Meadow Mouse. Abundant.

Evotomys, Coues.

E. rutilus (Pallas). Long-eared Mouse. Rather rare.

Calomys, Waterhouse.

C. Americanus (Kerr). Common White-footed Mouse. Dormouse. Common.

Mus, Linnæus. Old World Rats and Mice.

M. decumanus, Pallas. Brown or Norway Rat. Abundant.

M. rattus, L. Black Rat. Common.

M. musculus, L. Common House Mouse. Too abundant.

Family 63. CASTORID正. The Beavers.

Castor, Linnæus.

C. fiber, L. Beaver. Very rare. C. A. Downs. 
FAMily 64. SCIURID咂. The Squirrels.

Arctomys, Schreber.

A. monax (L.). Woodchuck. Ground Hog. Very common.

Tamias, Illiger.

T. striatus (L.). Chipmunk. Ground Squirrel. Abundant.

Sciurus, Linnæus.

S. Hudsonicus, Erxleben. Red Squirrel. Chickaree. Very common.

S. Carolınensis, Gmelin. Includes,-Gray Squirrel, common ; Black Squirrel, very rare.

Sciuropterus, Frédéric Cuvier.

S. volans (L.). Common Flying Squirrel. Rather common.

Order XXV. INSECTIVORA. The INsect-eaters.

FAMILY 65. SORICID瓜. THE SHREWS.

Sorex, Linnæus.

S. Forsteri (Richardson). Probable.

S. platyrhinus (De Kay). Common Shrew. Shrew Mouse. Not rare.

Blarina, Gray.

B. brevicauda (Say). Mole-Shrew. Probable.

B. angusticeps, Baird. Not rare.

Family 66. TALPID正. The Moles.

Scalops, Cuvier,

S. aquaticus (L.). Common Mole. Common.

Scapanus, Pomel.

S. Americanus (Bartram). Hairy-tailed Mole. Probable.

Condylura, Illiger.

C. cristata (L.). Star-nosed Mole. Common.

Order XXII. CHEIROPTERA. ThE BAts.

FAMILY 67. VESPERTILIONID无. ThE CoMmon BATS.

Vespertilio, Linnæus.

V. subulatus, Say. Little Brown Bat. Very common.

Vesperugo, Keyserling \& Blasius.

V. noctivagans (Le Conte). Silver Black Bat. Not common. R. Chellis.

V. Georgianus (F. Cuvier). Rare.

V. serotinus (Schreber), var. fuscus, Beauvais. Long-eared Bat. Rare.

Atalapha, Rafinesque.

A. Noveboracensis (Erxleben). Red Bat. Common.

A. cinerea (Beauvais). Hoary Bat. Possible. 
Order XyViI. Ungulata. The Hoofed Mammals.

FAmily 68. CERVID曆. The Deer.

Cariacus, Gray.

C. Virginianus (Boddaert). Virginia Deer. Red Deer. Rare. Has been reintroduced into Vermont, and may be seen occasionally throughout the district.

Order XXViII. FERÆ. The Flesh-Eaters or Carnivora.

Family 69. PROCYONID五. The Raccoons.

Procyon, Storr.

P. lotor (L.). Common Raccoon. Coon. Rather common.

FAMILY 70. URSID无. The BeARS.

Ursus, Linnæus.

U. Americanus, Pallas. Brown, Black, or Cinnamon Bear. Rare.

\section{Family 7i. MUSTELID洢. The Weasels.}

Mephitis, Cuvier.

M. mephitica (Shaw). Common Skunk. Too common.

Gulo, Storr.

G. gulo (L.). Wolverene. Probable.

Mustela, Linnæus.

M. Americana, Turton. Sable. Pine Marten. Not common.

M. Pennanti (Erxleben). Black Cat. Rare.

Putorius, Cuvier.

P. vison (Schreber). Mink. Rather common.

P. erminea (L.). Weasel. Ermine. Stoat. Rather common.

P. nivalis (L.). Least Weasel. Rare.

\section{FAMILY 72. CANID开. The Dogs.}

Vulpes, Brisson.

V. vulpes (L.). Red Fox. Rather common.

var. decussatus, Desm. The Cross Fox. Very rare.

var. argentatus, Shaw. Black or Silver Fox. Very rare.

Family 73. FELID床. The Cats.

Lynx, Rafinesque.

I. Canadensis (Desmarest). Canada Lynx. Rare.

L. rufus (Guldenstädt). American Wild Cat. Rare.

Felis, Linnæus. Cats.

F. concolor, L. American Panther. Cougar. Puma. Very rare. 
Order Xyix. Primates. The Anthropoid Mammals.

Family 74. HOMINID压. The Men.

Homo, Linnæus.

H. sapiens, L. Man.

var. Americanus, L. " American Indian."

var. Europæus, L. Caucasian race.

var. Asiaticus, L. Mongolian race.

var. Afer, L. Negro race.

Fishes,

SUMMARY OF SPECIES.

Batrachians,

Reptiles,

Birds,

Nammals,

19I

Total,

NoTE.-The above List, it is hoped, will furnish a good working basis for a thorough exploration of the region designated, and ultimately for a complete Catalogue of the Vertebrates occurring within our limits. The coöperation of all naturalists and sportsmen is invited, and they. are requested to report all additions, corrections, and information of any kind, to either PROF. T. W. D. Worthen or Prof. H. G. Jesup, Hanover, N. H. 



\section{INDEX TO PLANTS.}

[Synonym in Parentheses ; Cultivated Plants in Italics.]

Abies

Abutilon

Acalypha

Acer

Achillea

Aconitum

Acorus

Actiea

Adder's-mouth

Adder's-tongue

Adder's-tongue Family, 5

Adiantum

Adlumia

Esculus

A grimonia

A gropyrum

A grostis

(Aira)

Alder

Black

Alisma

Alismaceæ

Allium

Alnus

Alopecurus

Alyssum

A marantace

Amaranth Family

Amarantus

Ambrosia

Amelanchier

Ampelopsis

Amphicarpea

Anacardiaceæ

Anawallis

A naphalis

Andromeda

Andropogon

Anemone

Anemonella

Angelica

Antennaria

Authemis

Antloxanthum

Anychia

Apliyllon

Apios

Apium

Aplectrum

Apocynacea

A pocynum

Apple

Balsam
Crab
41 Apple of Peru

(Aquifoliacea)

Aquilegia

Arabis

Aracea

Arachis

Aralia

Araliacex

Arbor-vita

Arbutus, Trailing

Arcenthobium

Aretium

Arctostaphylos

Arenaria

Arethusa

Arisama

Aristida

Aristolochia

A ristolochiaceæ

Arrhenatherum

Arrow-head

Artemisia

Arum, Arrow Water

Arum Family

Asarum

Asclepiadaceæ

Asclepias

Ash

22

aragus

11 Aspen

10 Asphodel, False

27 Aspidium

2 Asplenium

6 Asprella

52 Aster

1,2 Astragalus

Avena

16 Avens

22 (Azalea)

42 J3arberry Family

27 Barley

27 Basil

14 Basswood

16 bean

14 Bearberry
30 Beard-tongue 30

Bedistraw 20

Bee-balm 32

Beech-drops 31

7 Beech 39

$\begin{array}{ll}\text { Blue } & 38 \\ \text { Beet, } & 3 t\end{array}$

Beggar's-lice 28

Bellflower 25

26 Bellwort 45

36 Berberidaceæ

23 Berberis

25 Bergamot

Beta

43 Betula

47 (Betulaceæ)

52 Bidens

36 Bindweed

36 kirch Family

5:3 Birthw ort

47 Birthwort Family

23 Bishop's-cap

47 Bitter-nut

47 Bittersweet

47 Climbing

36 Blackberry

27 Bladder-nut

7 Bladderwort Family

27 Blephilia

13 Blite, Strawberry

8 (Blitum)

45 Blood-root

39 Blueberry

46 Blue-curls

57 Blue-tlag

5f Bluets

55 Bohmeria

21 Boneset

11 Borage Family

54 Borragin aceæ

12 Botrychium

26 Box-elder

Brachyelytrum

40 Brake

8 ('lit)

2 Brasenia

4 Brassica

2 Bromus

2 liroom-rape Family

55 Brumella

32,33 Bnckbean

i Buclieye

12 Bucktiorn

25 Buckthorn Family
 
Buckwheat

Buckwheat Family

Buda

Bulrush

Bunch-berry

Burdock

Burnet

Burning-bush

Bur-reed

Buttercup

Butternut

Button-bush

Buttonwood

C'abbage

Cabbage, Skunk

Calamagrostis

Calamintha

Calla

Callitriche

Calopogon

Caltha

Calypso

(Calystegia)

Camelina

Campanulaceæ

Campanula Family

Camptosorus

Cancer-root

Candytuft

Cannabis

Caprifoliaceæ

Capsella

Caragana

Caraway

Cardamine

Cardiual-flower

Carex

Carpet-weed

Carpinus

Carrot

Carum

Carsa

Caryophyllaceæ

Cashew Family

Cassandra

Cassia

Castanea

('astor-oil plant

Ciatchfly

Catnip

Cat-tail Family

Caulophyllum

Ceanothus

Cedar, White Red

Celandine

Celastraceæ

Celastrus

Celery

Celosia

Celtis

Cenchrus

Cephalanthus

Cerastium

Ceratophyllaceæ

Ceratopliyllum

Claumonile

Checkerberry

Chelidonium

Cr elone

Chenopodiaceæ

Clienopodium

Cherry

Ground
35 Chess

35 Chestnut

6 Chickweed

49 Forked

17 Chicory

23 Chimaphila

13 Chiogenes

8 Chionanthus

47 Chives

2 Choke-berry

37 Chrysanthemum

20 Chrysopogon

37 Chrysosplenium

Cicliorinm

5 Cicuta

47 Cimicifuga

53 Cimna

32 Cinque-f̂oil

47 Circæa

15 (Cirsium)

43 Cistareæ

2 ('itrulus

42 Cladium

29 Claytonia

4 Clearweed

25 Clematis

25 Clintonia

57 Clover

31 Bush

5 Sweet

37 Club-moss

19 Club-moss Family

4 Cnicus

12 Cocklebur

17 Cocliscomb

4 Cohosh, Black

25

16 Coltsfoot

38 Sweet

16 Columbine

17 Comandra

38 Comfrey

6, 34 Compositæ

10 Composite Family

26 Cone-flower

11 Coniferæ

39 Conioselinum

37 Colium

6 Conopliolis

33 Convallario

47 Convolvulaceæ

2 Convolvulus Family

9 Coptis

41 Corallorhiza

41 Coral-root

3 Cornaceæ

8 Corn-cockle

8 Cormel

17 Cornus

34 Coronilla

37 Corydalis

52 Corylus

20 Cottonwood

6 Cowslip

40 Cranberry

40 High

23 (rassulaceæ

26 Cratægus

3 Cress

30 r'rocus

34 Crowberry Family

34 Crow foot Family

12 Crnciferæ

29 Cryptotænia
55 Cucumber 16

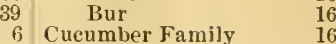

34 Cucumber-root, Indian 45

24 Cucumis 16

26 Cucurbita 16

25 Cucurbitaceæ 16

27 Cudweed

44 Cupuliferæ 38

13 Currant 14

23 Cuscuta

52 Cydonia 14

14 Cynoglossum 29

24 Cyperaceæ 48

17 Cyperus 48

2 Cypress-rine 29

3 Cypripedium 44

13 Cystopteris

16 Dactylis 54

24 Daisy, Ox-eye 23

16 Dalibarda 12

49 Dandelion 24

7 Danthonia 54

- Daphne 36

Datura 30

1,2 Daucus 16

10 Delphinium 2

11 Dentaria 4

10 Deschampsia 54

58 Desmodium 11

58 Dianthus 6

24 Dicentra $5 \frac{3}{57}$

22 Diervilla 19

34 lirca 36

2 Dock 35

46 Dodder Family 29

23 Dogwood Family 17

23 Dragon-head

2 Drosera. 15

8, 29 Droseraceæ 15

Duckweed Family 47

20 Dulichium 49

20 Dutchman's-breeches

41 Eatonia 54

16 Echinocystis 16

17 Echinospermum 25

31 Echium 29

44 Elder 19

29 Elecampane 22

29 Eleocharis 49

2 Elm 37

42 Elodes 7

17. Elymus

6 Enpetraceæ $\quad 40$

17, 18 Empetıum 40

17 Epigæa 26

12 Fpilobium 15

3 Epiphegus 31

35 Equisetaceæ $\quad 56$

40 Equisetum 56

2 Eragrostis 54

25 Erechlites 23

19 Fricacræ · 25

15 Erigeron 21

13 Eriocauleæ 48

4 Eriocanlon 48

44 Eriophorum 49

40 Erodium 8

1 Errsimum 4

4 Erythronium 45

17 Enonymus 
Eupatorium

Euphorbia

Euphorbiaceæ

Everlasting

Fagopyrum

Fagus

Fennel

Fern, Adder's-tongue

Beech

Bladder

Cluristmas

Cinnamon

Flowering

Grape

Lady

IIaiden-hair

Queen

Royal

Sensitive

shield

Sweet

Fern Family

Festuca

Fever-wort

Ficoidea

Figwort

Figwort Family

Filices

Fimbrystilis

Fir

Fireweed

Five-tinger

Flax Family

Fleabane

Floating-heart

Foniculum

Forget-me-not

Foxglove

Fragaria

Fraxinus

Frittillaria

Fringe-tree

Frog's-bit Family

Fumaria

Fumariaceæ

Fumitory Climbing

Fumitory Family

Funkia

Gialeopsis

Galingale

Galinsoga

Galium

Garlic

Gaultheria

Gaylussacia

Gentiana

Gentianacex

Gentian Family

Geraniacex

Geranium

Geranium Family

Gerardia

Germander

Geuni

Ginseng

Ginseng Family

vilarliolus

Gleditschia

Glyceria

Gnaphalium

Golden-rod

Gold-thread
20 Gomphrena

36 Good-King-Henry

36 Goodyera

22 Gooseberry

Goosefoot

35 Goosefoot Family

39 Gramineæ

1i Grape

58 Grass, Barnyard

Beard

Bent

Blue-eyed

Blue-joint

Bottle-brush

Brome

Bur

Canary

Cat's-tail

Cotton

Couch

Crab

Darnel

Drop-seed

Eel

Fescue

Fox-tail

Hair

Holy

Indian

June

Manna

Meadow

iillet

Momntain-rice

Oat

Old-witeh

Orchard

Panic

Poverty

Reed-bent

Ribbon

Rush

Rye

Spear

Sweet vernal

Tape

Thin

Timothy

Velvet

White

Wire

WTood-reed

Grass Family

Gratiola

Greenbrier

Gromwell

6 Ground Ivy

5 Ground-nut

Groundsel

Habenaria

8 Hackberry

Haloragea

8 II a mamelidex

31 IIamamelis

32 Hardhack

12 Harebell

17 Hawkweed

17 Hawthorn

44 Hazelnut

12 Hazel, Witch

54 Heath Family

2.) Herleoma

20 Hedge-hyssop

2 Heliantlemum
$3 \pm$ Helianthus

34 Heliopsis

43 Hellebore, False

14 Hemerocallis

$3 \pm$ Hemlock

34 Ground

51 Poison

Hemp Viter

Indian

Henbane

Hepatica

Heracleum

Herb-Robert

4 Hesperis

Hibiscus

Hickory

Hieracium

Hierochloe

Hippuris

Hobble-bush

Hog Pea-nut

Holcus

Holly

Mountain

52, 53 Holly Family

53,54 Hone-wort

52 Honeysuckle

Honeysuckle Family

54 Hop-tree

53, 5џ Hordeum

i3 Horehound

52

Hornbeam

53, 54 Hornwort Family

52 Horse-chestnut

54 Horsetail Family

51,52 Horse-mint

52 Horseradish

53 Horseweed

5. Hound's-tongue

53 Houstonia

55 Huckleberry

54 Humulus

52 Hyacinthus

42 Hydrocaridaceæ

53 Hydrocotyle

Hydrophyllaceæ

52,5 Hycophyllum

yoseyamus

4 Hypericaceæ

53 Hypericum

51 Hyssop

30 Hyssopus

44 Il,eris

29 Ilex

33 Ilicinea

Illecebraceæ

Ilysanthes

43

Impatiens

Indian-corn

Indian-pipe

India-wheat

15 Inula

15 Ipomere

12 Iriclacem

25 Ii is

Iris family

Ironwood

Isoetes

Ivy, Poison

Jurob's ladder

30 Jamestown-weed

Jewel-weed

\section{2}

22

44

17,41

41

17

37

27

1

16

8

19

19

37

55

33

38

40

9

56

21

28 


\begin{tabular}{|c|c|c|}
\hline Juglandaceæ & 37 & Loosestrife Family \\
\hline Juglans & $3 \overline{1}$ & Lopseed \\
\hline Juncus & 46 & Loranthaceæ \\
\hline Juncaceæ & 46 & Lousew ort \\
\hline June-berry & 14 & Lovage \\
\hline Juniper & 41 & Lucerne \\
\hline Juniperus & 41 & $\begin{array}{l}\text { Ludwigia } \\
\text { Lungucort }\end{array}$ \\
\hline líalmia & 26 & Lupinus \\
\hline linawel & 34 & Luzula \\
\hline Knotwort Family & 34 & Lycium \\
\hline Krigia & 24 & $\begin{array}{l}\text { Lychnis } \\
\text { Lycopersicum }\end{array}$ \\
\hline Labiatæe & 32 & Lycopodiaceæ \\
\hline Lactuca & 24 & Lycopodium \\
\hline Ladies -tresses & 42 & Lycopus \\
\hline Lady's-slipper & 44 & 14ysimachia \\
\hline $\begin{array}{l}\text { Lady's-thumb } \\
\text { Lagenaria }\end{array}$ & $\begin{array}{l}35 \\
16\end{array}$ & $\begin{array}{l}\text { Lythracea } \\
\text { Lythrum }\end{array}$ \\
\hline $\begin{array}{l}\text { Lagenaria } \\
\text { Lamium }\end{array}$ & $\begin{array}{l}16 \\
33\end{array}$ & Lythrum \\
\hline Laportea & 37 & Madder Family \\
\hline (Lappa) & 23 & Maianthemum \\
\hline Larch & 41 & Maize \\
\hline Larix & 41 & Mallow Family \\
\hline Larkspur & 2 & Malva \\
\hline Lauraceæ & 36 & Malvaceæ \\
\hline Laurel & $\frac{26}{36}$ & Mandrake \\
\hline $\begin{array}{l}\text { Laurel Family } \\
\text { Lavandula }\end{array}$ & $\begin{array}{l}36 \\
33\end{array}$ & $\begin{array}{l}\text { Maple } \\
\text { Maple, Ash-leaved }\end{array}$ \\
\hline $\begin{array}{l}\text { Lavandula } \\
\text { Lavender }\end{array}$ & $\begin{array}{l}30 \\
33\end{array}$ & $\begin{array}{l}\text { Maple, Ash-leaved } \\
\text { Mare's tail }\end{array}$ \\
\hline $\begin{array}{l}\text { Lavender } \\
\text { Leather-leaf }\end{array}$ & 26 & Marigold, Bur \\
\hline Leatherwood & 36 & M̂arsh \\
\hline Lechea & 5 & Water \\
\hline Ledum & 26 & Marjoram \\
\hline Leek, Wild & 44 & Marrubium \\
\hline Leersia & 52 & Matrimony-vine \\
\hline Leguminosæ & 10 & Matthiola \\
\hline Lemna & 47 & $\begin{array}{l}\text { May-flower } \\
\text { May-weed }\end{array}$ \\
\hline $\begin{array}{l}\text { Lemnaceæ } \\
\text { Lentibulaceæ }\end{array}$ & $\begin{array}{l}4 i \\
31\end{array}$ & \\
\hline $\begin{array}{l}\text { Lentibulaceæ } \\
\text { Leonurus }\end{array}$ & $\begin{array}{l}01 \\
33\end{array}$ & $\begin{array}{l}\text { Meadow-sweet } \\
\text { Medeola }\end{array}$ \\
\hline & 5 & Medicago \\
\hline Lespedeza & 11 & Medick \\
\hline Lettuce & 24 & Melampyrum \\
\hline Leverwood & 38 & Melilotus \\
\hline Ligusticum & 17 & Melissa \\
\hline Ligustrum & $2 \pi$ & Menispermum \\
\hline Lilac & $2 i$ & Menispermaceæ \\
\hline Liliaceæ & 44 & Mentha \\
\hline Lilium & 3. $44,45,46$ & $\begin{array}{l}\text { Menyanthes } \\
\text { Mercury, Three-seeded }\end{array}$ \\
\hline${ }_{\text {Lily }}$ & $\begin{array}{r}3,44,45,46 \\
44,46\end{array}$ & $\begin{array}{l}\text { Mercury, Three-seeded } \\
\text { Mertensia }\end{array}$ \\
\hline Sword & 44 & Mermaid-weed \\
\hline Tiger & 45 & Mezereum Family \\
\hline Water & 3 & Milkweed Family \\
\hline Lily Family & 44 & Milkwort Family \\
\hline Lily of the Valley & 44 & Milium \\
\hline Limnanthemum & 28 & Mimulus \\
\hline Linaceæ & $\begin{array}{r}8 \\
30\end{array}$ & Mint Family \\
\hline $\begin{array}{l}\text { Linaria } \\
\text { Linden Family }\end{array}$ & $\frac{30}{7}$ & $\begin{array}{l}\text { Mistletoe Family } \\
\text { Iitella }\end{array}$ \\
\hline $\begin{array}{l}\text { Linden Family } \\
\text { Lindera }\end{array}$ & 36 & $\begin{array}{l}\text { Mitella } \\
\text { Mitchella }\end{array}$ \\
\hline Linnea & 19 & Mitre-wort \\
\hline Linum & 8 & Muck-orange \\
\hline Liparis & 42 & Mollngo \\
\hline Listera & 42 & IIonarda \\
\hline Lithospermum & 29 & Moneses \\
\hline Liverwort & 1 & Moweywort \\
\hline Lobeliaceæ & 25 & Monkey-flower \\
\hline Lohelia Family & 11. 25 & $\begin{array}{l}\text { Monkshood } \\
\text { Monotropa }\end{array}$ \\
\hline $\begin{array}{l}\text { Locust } \\
\text { Loiseleuria }\end{array}$ & 11, 12 & $\begin{array}{l}\text { Monotropa } \\
\text { Moonseed }\end{array}$ \\
\hline Lolium & 5 & IInonseed Family \\
\hline Lonicera & 19 & Moonwort \\
\hline Loosestrife & 15,27 & Morning-glory \\
\hline
\end{tabular}

15 Morus 37

Iotherwort

36 Mountain-fringe 3

31
17 Muhlenbergia

11 (Mulgedium) 24

15 Iullein 30

29 Muscari 46

10 II ustard 4

46 Mustard Family 4

30 Mycrostylis 42

6 Iyosotis $\quad 29$

58 Mvricaceæ 38

58 Myriophyllum 15

32 (Nabalus)

5 Naiadaceæ 48

15 Naias

(Nardosmia) 23

19 Nasturtium 4

45 Negundo 9

55 Nemopanthes

7 Nettle 33, 37

Nettle Family 37

3 Nicandra 30

9 Nicotiana 30

9 Nightshade, Enchanters' 16

15 Nightshade Family 29

22 Nuphar

2 Nympliæa

23 Nymphæaceæ 3

32 Nyssa 15

Oak 38

5 Jerusalem 34

26 Oakesia 45

3 Oak Family 38

12 Ocimum 33

45 Enothera 16

11 Oleaceæ 27

12 Olive Family 27

31 ()nagraceæ 15

10 Onion 44

33 Onoclea 57

2 Ophioglossaceæ

2 Ophioglossum 58

3:- Orchidaceæ 42

28 Orchis 43

36 Orchis Family 42

29 Origanum 32

15 Ornithogalum 44

36 Orobanchaceæ 31

27 Orpine Family 15

10 Oryzopsis 52

53 Ostrya

30 Osmorrhiza 11

32 Osmunda $\quad 57$

36 Oyster-plant 24

21) Oxalis 22

14

14 Preonia

16 Panicum 51

32 Papaveraceæ 3

26 Parnassia 14

27 Parsiey 17

30 Parsley Family 16

2 l'arsnip 16, 17

27 Partridge-berry 20

2 P'ispalum 51

2 Pastinaca $16^{2}$

58 Pea 12

29 l'ea-nut 12 
Pear

Pearlwort

Pect-tree

Pedicularis

Pellæa

Peltandra

Pennyroyal

Pennywort, Water

Penthorum

Pentstemon

Peppergrass

Pepperidge

Peppermint

Pepper-root

Petasites

Phalaris

Phaseolus

Phegopteris

Philarlelphus

Phleum

Plilox

Phryma

Physalis

Physostegia

Phytolacea

Pliytolaccaceæ

Picea.

Pickerel-weed Family

Pig-nut

Pig-weed

Pilea

Pimpernel

Pine

l'ine-drops

Pine Family

Pine-sap

Pink Family

Pimus

Pinweed

Pipe-vine

Pipewort Family

Pisum

Pitcher-plant

Pitcher-Plant Family

Plane-tree Family

Plantaginaceæ

Plantago

l'lantain Family

Platana ceæ

Platanus

Plum

Poa

Podophylhum

Pogonia

Poke, Indian

Pokeweed Family

Polypodium

Polemoniaceæ

Polemonium

Polemonium Family

Polygala Family

Polygalaceæ

Polygonaceæ

l'olygonatum

Poly aron um

Pondweed

Pondweed Family

l'ontederia

Pontederiaceæ

I'oplar

Poppy Family

l'opulus

I'ortulaca

l'ortulacaceæ

Potamogeton
14 Potato

6 Potentilla

12 Poterium

31 Prenanthes

56 Primrose, Evening

47 Primrose Family

32 Primulaceæ

17 J'rince's-feather

15 I'rince's pine

30 l'rivet

5 Proserpinaca

18 l'runus

3.2 Ptelea

4 P'teris

2.3 l'terospora

52 I'ulse ramily

12 l'urslane

Purslane Family

14 Putty-root

53 l'ycrianthemum

28 P'vrola

32 Pyrus

3 Quercus

Quillwort

Quince

Radish

Ragweed

liagwort

37 Raunnculaceæ

27,31) lanunculus

41, 58 Raphanus

26 Raspberry

41 Rattlesnake-plantain

27 Ruttlesnake-weed

6 Rlımnaceæ

41 Rhanmus

5 Rheum

36 Rhododendron

49 (Khodora)

12 Rlumbarb

3 Rhus

3 Rhyuchospora

Ribes

33 Ricinus

33 Robinia

33 Rocket

37 liock-rose
Rock-rose Family

Rosa

Rosacer

Rose Family

Roubieva

Rubiaceæ

Rubus

Rudbeckia

Rue Family

Rue, Meadow

liumex

liush Family

Rush

Beak

Club

spike

Twig

Rutaceæ

liye

Wild

Sage

agina

Sagittaria

t. John's-wort Family

salicaceæ
30 Salix

13 Salria

13 Sambucus

24 Sandalwood Family

16 sandwort

27 Sanguinaria

27 Sanicula

35 Siantalaceæ

26 Sapindaceæ

27 saponaria

15 Sarracenia

12, 14 Sarraceniaceæ

8 Sarsaparilla

56 Sassafras

26 Satureia

10 Saxifraga

Saxifragaceæ

Saxifrage Family

42 scheuchzeria

32 Scilla

26 Scirpus

13,14 Scleranthus

Serophularia

38 Scruphulariaceæ

58 Scutellaria

14 Secale

Sedge

Sedge Family

3 Selaginella

1 Stlaginellaceæ

2 Selavinella Family

Selt-heal

12 Senecio

43 Senua

24 sericocarpus

9 setalia

9 Shad-bush

35 Slieep-berry

26 shepherd's-purse

26 sicyos

35 silene

10 sisy mbrium

49 Sisyrinchium

14 Sium

37 skullcap

11 Smartweed

4 Smilacina

5 smilax

5 snake-head

13 snakeroot

12 snowberry

Soapwort

$$
\text { Creeping }
$$

19 Soapberry Family

12 Solanaceæ

22 solanum

solidago

1 Solomon's-seal

35 Sonchus

46 (Sorghum)

46 sorrel

49 Sour-:-um tree

49 Sparganium

49 Spearmint

49 Siecularia

8 speedwell

55 Spergula

55 (Spergularia)

spice-wood

3 Spikenard

6 spintehia

47 spiraa

$i$ Spiranthes

39 spirodela

39 


Spleenwort
Sporobolus
Spring-beauty
Spruce
Spurge Family
Spurrey
Squirrel-corn
Stachys
Staff-tree Family
Staphylea
star-flower
star-of-Bethlehem
Starwort
Water
steironema
stellaria
Stoch
Stone-crop
Storks-bill
Stramonium
Strawberry
streptopus
sundew
Sundew Family
Sunflower
Sumach
Summer Savory
Sweet-chcely
Sweet-fern
sweet-flag
Sweet-gale
Sweet-gale Family
Sycamore
Symphoricarpus
Symphytum
Symplocarpus
Syringa
Tamarack
Tanacetum
Tausy
Taraxacum
Taxus
Tea, Labrador
New Jersey
Teucrium
Thalictrum
(Thaspium)
Thistle
Thlaspi
Thorn-tree

\begin{aligned} 56 & Thoroughwort \\ 53 & Thuya \\ 7 & Thyme \\ 41 & Thymelæaceæ \\ 36 & Thymus \\ 6,7 & Tiarella \\ 3 & Tiger flower \\ 33 & Tigridia \\ 8 & Tilia \\ 10 & Tiliacer \\ 27 & Toarl-Flax \\ 44 & Tobacco \\ 21 & Tobacco, Indian \\ 15 & Totieldia \\ 27 & Tomato \\ 6 & Tragopogon \\ 5 & Trefoil \\ 15 & Tick \\ 8 & Trichostema \\ 30 & Trientalis \\ 12 & Trifolium \\ 45 & Trillium \\ 15 & Triosteum \\ 15 & Triticum \\ 22 & Tsuga \\ 10 & Tulipa \\ 33 & Tumbleweed \\ 17 & Turwip \\ 36 & Indian \\ 47 & Tussilago \\ 38 & Twayblade \\ 38 & Twin-flower \\ 37 & Twist-foot \\ 19 & Typha \\ 29 & Typhaceæ \\ 47 & \\ \hline & Ulnus \\ & \end{aligned}

14, 27

$\begin{aligned} & \text { Umbelliferæ } \\ 41 & \text { Urtica } \\ 23 & \text { Urticaceæ } \\ 23 & \text { Utricularia } \\ 24 & \text { Uvularia } \\ 41 & \\ 26 & \text { Vaccinium } \\ 9 & \text { Valericu, Greek } \\ 32 & \text { Vallisneria } \\ 1 & \text { Venus's looking-glass } \\ 17 & \text { Veratrum } \\ 29 & \text { Verbascum } \\ 4 & \text { Verbena } \\ 13 & \text { Verbenaceæ }\end{aligned}$

\begin{tabular}{|c|c|c|}
\hline & & \\
\hline 20 & Veronica & 31 \\
\hline 41 & Vervain Family & 32 \\
\hline 33 & Vetch & 11 \\
\hline 36 & Viburnum & 19 \\
\hline 33 & Vicia & 11 \\
\hline 14 & Vincetoxicum & 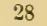 \\
\hline 44 & Vine Family & 9 \\
\hline 44 & Yiola & 5 \\
\hline I & Violaceze & \\
\hline 7 & Violet Family & \\
\hline 0,36 & Virginian creeper. & 9 \\
\hline 30 & Virgin's-bower & \\
\hline 25 & Vitaceæ & \\
\hline $\begin{array}{l}46 \\
30\end{array}$ & Vitis & \\
\hline 24 & Wake-robin & 45 \\
\hline 10 & Walking-leaf & 0 \\
\hline 11 & Wildsteinia & 12 \\
\hline 32 & Wrainut Family & 37 \\
\hline 27 & Waterleaf Family & \\
\hline 10 & Water-lily Family & \\
\hline 45 & Water-mílfoil Family & 15 \\
\hline 19 & Water-plantain Family & 47 \\
\hline $5 \overline{5}$ & Water-shield & \\
\hline 41 & Waterweed & 42 \\
\hline 46 & Wheat & 55 \\
\hline 34 & Cow & 31 \\
\hline 5 & Wild Ginger & 36 \\
\hline 47 & Willow & 39 \\
\hline 23 & Willow Family & 39 \\
\hline $4: 2$ & Willow-herb & \\
\hline 19 & Wind-flower & 1 \\
\hline 45 & Wintergreen & 26 \\
\hline 47 & Wistaria & 13 \\
\hline 47 & Witch-hazel Family & 1 \\
\hline & Woodbine & \\
\hline 37 & Woodsia & 57 \\
\hline 16 & Wormwood & \\
\hline 37 & Tanthium & 22 \\
\hline 31 & Xanthoxylum & \\
\hline 45 & Xyridaceæ & $\begin{array}{l}46 \\
46\end{array}$ \\
\hline & Tyris & \\
\hline $\begin{array}{l}25 \\
28\end{array}$ & Yarrow & \\
\hline 42 & Yellow-eyed-gr & \\
\hline 25 & $\begin{array}{l}\text { 1 ew } \\
\text { Yucea }\end{array}$ & 6 \\
\hline 30 & Zanuichellia & 48 \\
\hline 32 & $Z e a$ & 0 \\
\hline 32 & Zizia & \\
\hline
\end{tabular}




\section{NDEX TO VERTEBRATES.}

Acanthis

Acanthopteri

Accipiter

Acris

Actitis

Adder

Egialitis

Agelaius

Aix

Alau didæ

Alcedinidæ

Alcidæ

Alle

Anblystoma

A mblystomatidæ

Ameiurus

Ammodramus

Ampelidæ

Ampelis

Allas

Anatidæ

Anguilla

Anguillidæ

Anseres

Anthus

Antrostomus

Apodes

Aquila

Archibuteo

Arctomys

Ardea

Ardeidæ

Arenaria

Arvicola

Asio

A talapha

Auk

A res

Bartramia

Bass

Bat

Batrachia

Bear

Beaver

Bittern

Blackbird

Black Cat

Blarina

Blue Bird

Bobolink

Bob-white

Bonasa

Botaurus

Branta

Brown Thrasher

Bubo

lubbonidæ

Bufo

Bufonidæ

Bull-head

Buteo

\begin{tabular}{ll}
72 & Butcher-bird \\
60 & \\
68 & Calcarius \\
61 & Calomys \\
67 & Canidæ \\
63 & Caprimulgidæ \\
67 & Cariacus \\
72 & Carnivora \\
66 & Carpodacus \\
71 & Castor \\
69 & Castoridæ \\
65 & Cat-bird, \\
65 & Cat-fish. \\
61 & Catostomidæ \\
61 & Catostomus \\
\hline
\end{tabular}

59 Cedar-bird,

72, 73 Centrarchidæ

i4 Ceophloeus

74 Certhia

66 Certhiidæ

66 Cervidæ

60 Ceryle

60 Chætura

66 Charadriidæ

75 Chelidon

70 Chelopus

60 chelydra

68 Clielydridæ

68 Chewink

79 Chickadee

66 Chimney swallow

66 chipmunk

67 C'hiroptera

78 chordeiles

69 Chorophylus

79 Clirysemys

65 Chub Eel

65 Circus

Cistothorus

67 Clivicola

60 Coccyges

79 Coccyzus

61 Colaptes

80 Colinus

78 Colubridæ

66 Columbæ

71, 72 Columbidæ

80 Colymbus

i9 Compsothlypis

ii Condylura

i1 Contopus

67 Cormorant

68 Coot

66 Corvidæ

66 Corvus

76 Cottidx

69 Cottus

69 Cow-bird

61 Crane

61 Creeper

59 Crossbill

68 Crotalidæ
74 Crotalus 63

Crow

2 Cuckoo

is Cuculidæ

80 Cranocitta

70 Cyprinidæ

80

80 Dace

72 Darters

8 Deer

i8 Dendragapus

76 Dendroica

59 Diadophis

59 Diemyctylus

59 Dipper-duck

74 Diving-birds

60 Dolichony $x$

7) Dove

76 Dryobates

if Duck

so

69 Nagle

70 Ectopistes

67 Eel

73 Eel Pout

64 Empidonax

63 kmydidæ

63 Erethizon

73 Erismatura

66 Esocidæ

70 Esox

79 Etlieostoma

79 Eutainia

70 Eventognathi

62 Evet

64 Evotomys

60

68 Falco

76 Falcon

74 Falconidæ

69 Felidæ

69 Felis

in Feræ

67 Fiber

63 Finch

68 Flycatcher

68 Fox

65 Fringillidæ

75 Fro?

79 Tree

71 Fulica

65

67 (iadidæ

il Galeoscoptes

i1 fialline

60 Galliuago

60 (ieothlypis

i1 (ilires

66 Golden Robiu

74, if Goldfinch

72 Goose

63 (ioshawk 
Grebe

Grosbeak

Grouse

Gull

Gulo

Habia

Haliaëtus

Haplomi

Hare

Harporhynchus

Hawk

Hedyehoy

Helminthophila

Herodiones

Heron

Heterodon

High-holer

Hirundinidx

Hominidæ

llomo

Horn-pout

Humming-bird

Hyla

Hylidæ

Hystricidæ

Icteridæ

Icterus

Indian Hen

Indi : o-bird

Insectivora

Isospondyli

Jay

Jumping Mouse

Junco

Kiildeer

King-bird

lingtislier

li inglet

Laniidæ

Lanius

Lapland-longspur

Laridæ

Lark

Larus

Lepomis

Leporidæ

Lepus

Limicolæ

Linpeltis

Longipennes

Loon

Lophodytes

Lota

Loxia

Lynx

Macrochires

Manmmalia

Man

Marsh-Harrier

Martin, I’urple

Meadow-lark

Mrgascops

Melauerpes

Melospiza

Mephitis

Merganser

Merula

Mice

Micropodidæ
65 Micropterus

72,73 Miller's Thumb

67, 68 Mimus

65 Mink

8J Minnow

Mniotilta

73 Mniotiltidæ

68 Mocking-bird

60 Mole

78 Molotlirus

76 Motacillida

68, 69 Ind Hen

78 Muridæ

74 Mus

66 Muskrat

66 Mustela

63 Mustelidæ

70 Myiarchus

73

81 Nematognatli

81 Newt

59 Nigint-hawk

70 Notemigonus

62 Notropis

61 Nuthatch

78 Nyetala

Nyctea

i1 Nycticorax

72

66 Ophibolus

73 Oplidia

-9 Uriole

60 Osprey

Otocoris

71 Oven-bird

$78 \mathrm{Ow}$

73

Paludicolæe

67 Pandion

71 Panther

69 Paridæ

i6 Partridge l'arus

74 Passer

74 Passeres

72 I'asserella

65 Passerina

71, 75 l'elobatidæ

65 Perca

60 l'erch

is Percidr

is l'erisoreus

67 Petrochelidon

63 Pewee

65 Phalacrocoracidæ

65 P'halacrocorax

66 I'lilohela

60 Phobe

72 Pici

80 Picidæ

Pickerel

70 P'icoides

is P'igeon, Wild

s1 Jike

68 l'ine-fincl

73 Pine-marten

7:2 Pinicola

69 J'ipilo

i0 l'iranga

T3 l'isrees

So Plectroplienax

66 l'lethodon

7 l'lethodontida

7\&, 79 Pleurodelida

70 Plover
60 Podiceps 65

60 l'odicipidæ

76 Podilymbus

80 Poocætes 72

59 Porcupine 78

it Porzana 67

it Primates 81

if l'rocyon 80

79 Procyonidæ 80

71 l'rogne 73

5 J'mmpkin.seed 60

67 Purple-finch i2

78 l'utorius 80

is Pygopodes 65

78

80 Quail

80 Quiscalus

Rabbit

9 Raccoon

61 Rail

T0 Rallidæ

59 Rallus

59 Rana

76 Kanidæ

69 Raptores

69 Rat

66 Rattlesnake

Red-breast

63 Red-poll-linnet

63 Pedstart

72 Regulus

69 Reptilia

71 Rlinichthys

75 Robin

69 Rodents

$72,73, \frac{5}{7}$

66 Sable 80

69 salamander 61

80 salientia

76. Salmonidæ

68 salmon-trout

76 salvelinus

72 sand-martin

i1 Sandpiper

73 sap-sucker

i3 sayornis

61 Scalops

60 Scapanus

60 Scapliopus

60 Sciuridæ

71 Sciuropterus

73 Sciurus

71 Scolecophagus

65 Scolopacidæ

65 Scotiaptex

67 Sculpin

71 Seiurus

70 Semotilus

70 retopliaga

60 Sheldrake

(1) Shiner

68 Shrew

60 Shrike

i2 sialia

80 Siluridæ

72 sitıa

13 skuık

73 snake

59 silipe

72 silow-bird

61 snow-bunting

61 sorex

61 Foricidx

67 spade-foot
78

$66,+50$

66

66

62

65

63

71

75

76

61

61
60

60

60

74 
INDEX.

Sparrow

Spelerpes

sphyrapicus

Spinus

Spizella

Squirrel

Squawk

Sterna

Stizosterlion

storeria

Sturnella

sucker

Sunfish

Surnia

Swallow

Swift

Sylvania

sylviidæ

Syrnium

Tachycineta

Talpidæ

Tamias

Tanager

Tanagridæ

Tattler

Teal

Tell-tale

Tern

\begin{tabular}{rl|l}
72,73 & Testudinata \\
61 & Tetraonidæ \\
70 & Thrusl \\
72 & Tip up \\
73 & Titlark \\
79 & Titmouse \\
66 & Toad \\
65 & Tree \\
60 & Tortoise \\
63 & Totanus \\
72 & Tringa \\
59 & Trochilidæ \\
60 & Trochilus \\
69 & Troglodytes \\
73,74 & Troglodytidæ \\
70 & Tropidonotus \\
75 & Trout \\
76 & Turdidæ \\
69 & Turdus \\
73 & Turnstone \\
79 & Tyrtle \\
79 & Tyranidæ \\
73 & Urannus \\
73 & Ungulata \\
67 & Urinator \\
66 & Trinatoridæ \\
67 & Trodela \\
65 & Ursidæ
\end{tabular}

80

79

79

79

$-14$

s0

$74,-5,-60$

Warbler $74,75,76$

i0. Water-wagtail 75

0 W $\mathrm{ux}$-wing

6 Weasel 80

i6 Whippoorwill 70

63 IVild-cat 80

60 Wolverene 80

76 Woodehuck 79

76,7 Woodeock

67 Woodpecker 70

63, 64 Wren 76

71 Yellow-bird $\quad 72$

Yellow-hammer $\quad 70$

80 Yellow-throat

65 Zapodidx 78

6.) Zapus

80 Zonotrichia 






